Illinois State University

ISU ReD: Research and eData

Theses and Dissertations

7-28-2021

\title{
Optimizing University Campuses for Learning, Wellbeing and Equity: an Applied Study of Higher Education Facilities Organizational Systems
}

Melissa Nergard

Illinois State University, mnergard@gmail.com

Follow this and additional works at: https://ir.library.illinoisstate.edu/etd

\section{Recommended Citation}

Nergard, Melissa, "Optimizing University Campuses for Learning, Wellbeing and Equity: an Applied Study of Higher Education Facilities Organizational Systems" (2021). Theses and Dissertations. 1500.

https://ir.library.illinoisstate.edu/etd/1500

This Dissertation is brought to you for free and open access by ISU ReD: Research and eData. It has been accepted for inclusion in Theses and Dissertations by an authorized administrator of ISU ReD: Research and eData. For more information, please contact ISUReD@ilstu.edu. 
OPTIMIZING UNIVERSITY CAMPUSES FOR LEARNING, WELLBEING AND EQUITY:

AN APPLIED STUDY OF HIGHER EDUCATION FACILITIES ORGANIZATIONAL

SYSTEMS

\section{MELISSA A. NERGARD}

\section{Pages}

College and university campuses are designed and maintained by experts in facilities management and planning. What is often lacking in these processes is expertise in the science of designing learning environments which also promote health and inclusivity. University organizational structures aid in the autonomy of academic freedom and the nimbleness of research endeavors but hinder operational effectiveness of the campus as a learning and research environment. A more integrated approach, which optimizes the knowledge inherent at a university, can mitigate the unintended conflicts that develop when campus design and maintenance is situated in the organizational structure as an administrative support function with operational missions and performance targets that are separated from learning, equity, and wellbeing. This paper examines the organizational structures of higher education and demonstrates how optimization through a systems approach can mitigate the adverse consequences of autonomy and decentralization in the design and operation of higher education campuses. Several examples of applied systems approaches will be analyzed for effectiveness and contributions to the core mission of higher education. 
KEYWORDS: sustainability; higher education; facilities; learning environment; process improvement; systems approach; systems optimization; organizational structure; campus design; resilience; financial stewardship; fiscal accountability; facilities management; capital planning; physical plant; classroom design; learning spaces; design for equity; design for learning; design for health; design for wellbeing 
OPTIMIZING UNIVERSITY CAMPUSES FOR LEARNING, WELLBEING AND EQUITY: AN APPLIED STUDY OF HIGHER EDUCATION FACILITIES ORGANIZATIONAL SYSTEMS

\author{
MELISSA A. NERGARD
}

A Dissertation Submitted in Partial

Fulfillment of the Requirements for the Degree of DOCTOR OF PHILOSOPHY

Department of Educational Administration and Foundations

ILLINOIS STATE UNIVERSITY 
Copyright 2021 Melissa A. Nergard 
OPTIMIZING UNIVERSITY CAMPUSES FOR LEARNING, WELLBEING AND EQUITY: AN APPLIED STUDY OF HIGHER EDUCATION FACILITIES ORGANIZATIONAL SYSTEMS

MELISSA A. NERGARD

COMMITTEE MEMBERS:

John Rugutt, Chair

Dianne Renn

Touré Reed

James Applegate 


\section{ACKNOWLEDGMENTS}

Learning is not a static process. It requires a great deal of change, and I would like to thank the people and organizations that enabled, and participated, in this learning endeavor. I will begin by thanking my committee, Dr. John Rugutt, Dr. Dianne Renn, Dr. Touré Reed, and Dr. James Applegate. Your expertise and experience in advising me through a process that I will likely undergo only once in my life, was simply invaluable. Your patience and thoughtful guidance on an unconventional and emerging field of practice helped me through many quandaries and will hopefully impact the learning environments of students for many years to come.

I have been privileged to have extraordinary mentors in university Facilities departments. People that have a passion for learning environments and serving students, especially Mike O'Grady, Steve Lancaster, and Bob McMains. I also need to thank my colleagues at the University of Wisconsin-Madison for being such amazing advocates and supporters of the process changes. They clearly see the same opportunities, and together we gained remarkable knowledge while initiating substantive and positive changes. I would particularly like to thank Kip McMahan, Jay Bieszke, Patrick Kass, Wendy Von Below, Dr. Cathy Middlecamp, Dr. Andrea Hicks, Laurent Heller, David Darling, and Rob Cramer for their willingness to critically examine how a university works and explore new approaches.

Any endeavor that requires this much time, work and participation obviously imposes change on others, and there have been so many that have sacrificed to help me achieve this degree. My partner Rory, and my children Erika and Lorien, gave up so much and completely revamped their lives for me. My parents, Tom and Ginger Shallow, instilled an endless curiosity 
and desire to learn, and gave up many opportunities to spend precious time together. Numerous friends and colleagues, particularly Dr. Joan Brehm, Dr. Tracy Harvey, Dr. Nathan Jandl, David Goebig, and Bart Lytel, provided endless encouragement and support. A special thanks to my K9 team, and my K9 partners Brennan and Epona, for weathering my absences - it truly takes a high performing team to ensure that we never falter in our mission and to unfailingly support each other.

My gratitude and appreciation are beyond my ability to articulate. I am humbled by your sacrifices and support. 


\section{CONTENTS}

Page

ACKNOWLEDGMENTS

CONTENTS

TABLES Vi

FIGURES

CHAPTER I: SILOES AND SYSTEMS 1

Introduction to the Problem and its Underlying Framework $\quad 1$

Background of the Problem $\quad 2$

$\begin{array}{ll}\text { Statement of the Problem } & 10\end{array}$

$\begin{array}{ll}\text { Research Questions } & 11\end{array}$

$\begin{array}{ll}\text { Purpose and Significance of the Study } & 12\end{array}$

$\begin{array}{ll}\text { Limitations and Assumptions } & 15\end{array}$

$\begin{array}{ll}\text { Definition of Terms } & 18\end{array}$

$\begin{array}{ll}\text { Organization of the Study: Conceptual Framework } & 20\end{array}$

CHAPTER II: REVIEW OF THE LITERATURE 22

$\begin{array}{ll}\text { Background } & 22\end{array}$

Facilities and Learning: Relationship and Impact 25

Attributes and Their Impact on Learning, Wellbeing, and Equity 26

Code Compliance, Social, Physiological and Health Imperatives in Campus Design 28

$\begin{array}{ll}\text { Applied Systems Thinking } & 30\end{array}$

Organization of Higher Education $\quad 33$ 
Evolving Conversations in Higher Education Systems: Transdisciplinary Practices

Summary of Scholarship on the Knowledge Enterprise and Transdisciplinarity 40

CHAPTER III: METHODS OF ANALYZING ORGANIZATIONAL PROCESSES 42

The Study Proposal $\quad 42$

Statement of Positionality $\quad 42$

$\begin{array}{lr}\text { Research Approach } & 46\end{array}$

$\begin{array}{lr}\text { Research Questions } & 48\end{array}$

$\begin{array}{lr}\text { Applied Research Methodology } & 48\end{array}$

$\begin{array}{ll}\text { The Researcher } & 50\end{array}$

The Applied Pilot Studies $\quad 52$

Incorporating Design Principles into the Capital Planning Process 55

Learning From Others $\quad 58$

$\begin{array}{lc}\text { Trial and Error: The Implementation and Analysis } & 60\end{array}$

$\begin{array}{ll}\text { Study Process } & 61\end{array}$

$\begin{array}{lc}\text { Types and Sources of Data } & 63\end{array}$

$\begin{array}{ll}\text { Research Method } & 65\end{array}$

$\begin{array}{ll}\text { Summary } & 66\end{array}$

CHAPTER IV: MAKING SENSE OF SYSTEMS

$\begin{array}{ll}\text { Introduction } & 68\end{array}$

$\begin{array}{lc}\text { Findings and Results } & 68\end{array}$

Organizational Structures: State to Public University 68

Applied Studies: Leveraging Organizational Systems for Campus Design $\quad 78$ 
Forensic Mapping, Identifying Integration Opportunities

$\begin{array}{ll}\text { Stakeholders and Design Criteria } & 84\end{array}$

Integration Pilots 1 and 2: Preliminary Design $\quad 89$

Integration Pilot 1 and 2: Evaluation $\quad 96$

Integration Pilot 3a: Informal Learning Spaces 97

Integration Pilot 3b: Designing Classrooms for Learning, Wellbeing, and Equity 102

Integration Study 4: Design for Equity, Lighting and Accommodations 107

Integration Pilot 5: Aligning Project Priorities at the Institutional Level 114

$\begin{array}{ll}\text { Assessment and Iteration } & 119\end{array}$

Examining the Metrics and Indicators $\quad 119$

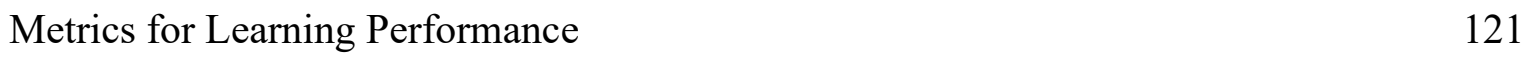

Facilities Departments and Professional Development $\quad 128$

$\begin{array}{ll}\text { Summary } & 132\end{array}$

CHAPTER V: CREATING WALKWAYS IN THE SKY: BRIDGING THE SILOES 134

$\begin{array}{ll}\text { Introduction } & 134\end{array}$

$\begin{array}{ll}\text { Summary of Findings } & 135\end{array}$

$\begin{array}{ll}\text { Implications in Practice } & 144\end{array}$

$\begin{array}{ll}\text { Recommendations for Research and Practice } & 148\end{array}$

$\begin{array}{ll}\text { Conclusion } & 152\end{array}$

$\begin{array}{ll}\text { REFERENCES } & 154\end{array}$ 


\section{TABLES}

Table $\quad$ Page

1. Comparison of Key Performance Indicators for Education and Facilities 120

2. Baseline Functionality Assessment of Spaces for General Classrooms 123

3. State of Wisconsin's Outcomes-Based Metrics 124

4. Curricula for APPA's Body of Knowledge (BOK) 130

5. Design Summary Checklist for Equitable Communities 141

6. Example of Metrics and Attributes for a Mother's Room 142 


\section{FIGURES}

Figure $\quad$ Page

1. Examples of an Elementary and University Classroom 5

2. Capital Project Process Swimlane Diagram $\quad 54$

3. Capital Budget Process Timeline 55

4. Example Display of UW-Madison STARS Academic Rating Comparisons 60

5. Vertical Hierarchies of the Capital Project and Budget Process 70

6. University of Michigan Capital Project Process 76

7. Example of Four Approval-Based Planning Scenarios for a Capital Project 77

8. Relationship Between Adaptive and Traditional Organizational Systems 79

9. University of Wisconsin-Madison's Design Principles 91

10. Veterinary Medicine Addition's Design Principle Charette 93

11. Signage for Informal Spaces Study 99

12. QR Code Survey for Each Piece of Test Furniture $\quad 100$

$\begin{array}{ll}\text { 13. Example of Seating Placed in Informal Spaces } & 101\end{array}$

14. Graphic Visualizations and Representations of Systems Approaches 145 


\section{CHAPTER I: SILOES AND SYSTEMS}

\section{Introduction to the Problem and its Underlying Framework}

Colleges and universities invest significant financial and human resources into their physical campuses through their Facilities departments, but often fail to leverage the academic and research enterprises in a manner that would optimize the environment in alignment with their missions. This results in ineffective and inefficient environments that subsequently impede and conflict with educational outcomes. This dissertation examines university organizational structures and the processes that direct and influence the design and operation of the physical campus environment. Some sectors of education, particularly elementary schools, incorporate many attributes that have been identified as contributing to learning, including aspects of student health, equity, and wellbeing. Yet as students advance through the educational system, the learning environments are less and less reflective of the attributes that positively impact learning, wellbeing, and equity. By the time students reach post-secondary education, the classrooms and informal learning spaces are largely devoid of color, warmth, comfort, a sense of belonging or sources of inspiration (see Figure 1). This applied research study is specifically analyzing how organizational relationships and processes within a public university of very high research activity, enable or inhibit the design considerations prioritizing learning, wellbeing, and equity into its campus facilities and landscapes. The objective of this analysis is to identify how processes related to campus facilities can be optimized to serve the core missions of learning and research.

My applied studies were informed by knowledge and experience that typical Facilities organizations have two to three different and distinct groups that deal with buildings and grounds 
- one group which deals with new buildings, large projects, and campus strategic planning (a capital planning department), and a second group which deals with small renovations, operations, and maintenance (a physical plant department), and a third which deals with utilities and utility infrastructure (a utility or energy department). The campus grounds and natural areas may fall under the purview of any of the three, with varying degrees of control and influence. One of the purposes of the study was to examine the organizational structures and processes of these three distinctive groups to determine how they communicate and cooperate, as well as to examine how each of them engage with the larger institutional enterprise. There are alternate organizational models in use by doctoral research institutions, and I examined a comparable peer to compare the effectiveness and alignment with a systems approach.

\section{Background of the Problem}

A university campus requires significant financial, environmental, and human resources. For the fiscal year 2019-2020, the University of Wisconsin-Madison had combined income revenues of $\$ 3.1$ billion. In that same year, the Facilities expenditures exceeded $\$ 245$ million, approximately $7.2 \%$ of the budget. When ranked against the expenditures for the 17 academic units, the Facilities department ranked $3^{\text {rd }}$, behind the School of Medicine and Public Health (\$606.6 million) and the College of Letters and Sciences (\$404.6 million) (UWMadison, Budget in Brief, p. 12, 2020). Within university organizational structures, the Facilities operations are responsible for the design and maintenance of the campus and are often organizationally, and in practice, detached from the mission-centric decision-making processes of the education and research enterprise. As the COVID-19 pandemic of 2020 has demonstrated however, teaching can be done virtually and at scale, and this may appear to 
support the untethering of facilities from the educational mission. Campus facilities were abandoned as they were ill-prepared to respond to a disruption of such magnitude. Although the efficacy of the massive on-line instructional endeavor has yet to be determined, what has become apparent is the impact that the environment plays in learning, wellbeing, and equity (Earthman, 2002). While the study of these relationships is not new, what has changed is the level of awareness of how critical the learning environment is to higher education - especially now that the prevalence of learning environments are outside the control of the institution (Clauson, 2018; Freeman, et. al, 2014; Giglotti, 2020). As virtual classes commenced in earnest in the Spring of 2020, we were given unparalleled access through Zoom windows into hundreds of thousands of individual learning spaces (some designed and some adapted for a crisis), and the majority of them did not look like the bland, institutional classrooms that had been so hurriedly abandoned.

When I first began this applied research study, I used the term learning environment broadly to refer to the built environment, landscaped and natural areas of a higher education campus. However, in the spring semester of 2020, the learning environments transformed from the classroom to the basement, the kitchen table, the bedroom, or even the car. Access to exterior spaces, whether they were backyards or community parks, transformed into areas of respite and allowed for safe distancing as a haven from a virus. These natural or landscaped exterior areas, adjacent to our built environment, also provided oft overlooked opportunities to expand curricular instruction and conduct laboratory assignments. When residence and dining halls were closed, the kitchens in the family home or off-campus apartments replaced them, yet for some students the closing of residence and dining halls resulted in homelessness and 
hunger. A campus serves many needs related to research, teaching and learning, and it fulfills social and physical aspects of wellbeing, yet the design and operations of the campus environment are not well integrated with the academic, research and student service enterprise.

There are challenges in coordinating the design of all the elements that are critical to the mission of a university, but it is not infeasible. The optimization of the human designed environment has exemplars in other education, municipal and corporate sectors. This dissertation will explore the unique organizational systems within higher education and how the processes in place to design and maintain the campus can impact the mission. I will examine how the research and knowledge developed in higher education is, or is not, integrated into the campus design and operations. Using an organizational systems approach to optimize effectiveness is shown to have significant benefits, and co-benefits to achieving mission-centered outcomes (Schein, 2017). Although the metrics for measuring many types of success are traditionally financial, this research intends to demonstrate how the valuation of co-benefits can transform decision-making processes so that they are inclusive of the campus users most impacted by the campus environment, yet generally allowed the least input into their design and operation. For example, the design and use of a classroom is a common and vital process to any campus. There are examples of good, coordinated processes for designing complex learning environments, however if the process is missing the input of instructors, students, accommodation services, or experts in learning environments, it is likely that the design will have missed several critical components that could optimize classrooms for learning, teaching, equity, and wellbeing. This creates unintended conflicts which hinder teaching and learning, exacerbate health disparities, and contribute to social inequities. 
Figure 1

Examples of an Elementary and University Classroom
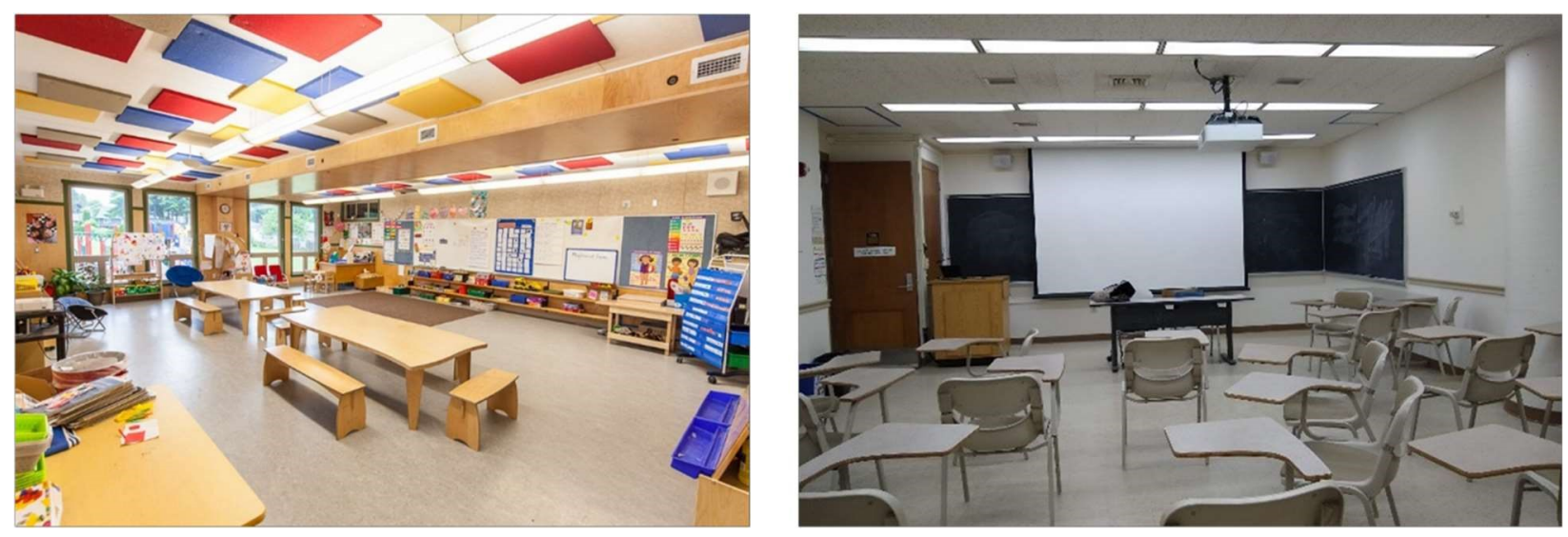

Note. Examples of the differences in classrooms from kindergarten to post-secondary, with an acknowledgement there is great variability in classrooms throughout the educational sector. The photo on left is of a kindergarten classroom (reprinted with permission from naturallywood.com). The photo on the right is a general use classroom at an R1, public university (own photo).

An analysis of spaces designated as classrooms can note what may seem to be small, nuanced failures of systems integration - such as designing seating for one body type (Holmes, 2018). But under pressure and over time these processes breakdown and expand in scope and impact (Jackson, 1982). This is evidenced by recent disjointed and lagging institutional responses during natural disasters, pandemics, and extreme violence (AAUP, 2007; Gigliotti, 2020). It is also visible by the sheer number of lecture halls and classrooms that were designed and built decades ago to serve a singular type of student, with a singular type of teaching. Instructional modes were limited, often with lectures being taught by the 'sage on the stage,' 
but these traditional lecture spaces are wholly inadequate to serve students with laptops, calculators, and smart phones, and they cannot support different pedagogies. The fixed, narrow seating with tiny tablet arms serves neither a physically diverse student body, the technology, nor the psychology and physiology of cognition. These types of spaces are not relics of the past. Classroom seating continues to fall short of serving students. Even the construct that a student must sit to learn demonstrates a hurdle to equitable higher education learning environments (Chrisman, Reddy, \& Purdy, 2020). The prevalence of these types of spaces, and the persistence of the processes that lead to them, are indicative of the inefficiencies and vulnerabilities that occur due to decentralized organizational structures in universities. This dissertation analyzes process interactions in higher education and proposes a sustainable systems approach to implementing enterprise level solutions, with a particular focus on the relationship between an institution's mission and its campus.

The seating example demonstrates some of the processes that institutions often address in linear ways. Pedagogies, classroom furniture and technology, accommodation services, class scheduling, maintenance, cleaning, and facilities design are undertaken as linear processes, and when they are not coordinated and integrated, they create conflict and inefficiencies. Similar scenarios of decentralized processes exist in research, student life, financial aid, community engagement, and in countless other functions. My research adopts a systems approach to creating environments which promote learning, positively affect health and wellbeing, and are inclusive - while also addressing the standards of efficient and effective high performing buildings. I will also examine why this particular focus on building performance is integral to the process. At colleges and universities, the processes must also 
maintain the divisional autonomy and academic freedom that is the hallmark of higher education in the United States.

What became apparent to me while reviewing the core scholarship on this topic is that the knowledge for systemic solutions exists and has largely been developed by academicians (Bertalannfy, 1972; Clegg, Josserand, Mehra \& Pitsis, 2016; Crow \& Dabars, 2015; Evans, 2015; Jahn, 2008; Keeling, Underhile \& Wall, 2007). What is missing is the application of that knowledge within the higher education organizations that house those very same scholars. Institutions are renown for being siloed, meaning that the disciplines and departments act autonomously and that there are not well established and coordinated pathways to facilitate collaboration along a central and overarching vision for a campus.

A 2019 study conducted by Huron, the American Council on Education (ACE), and the Georgia Institute of Technology notes that "Many institutions subscribe to planning models that were built for a different time..." and the legacies of those planning models have three immediate implications. The first is that the processes and planning that founded the institutions are now deeply and inextricably embedded. The second is that the cultures that have grown around these legacy planning models have developed into their own fiefdoms. A third, further reaching implication, is that institutions are not agile or flexible enough to respond quickly to a rapidly changing world and society. My observations of the implications for campus design and planning are buttressed by repeated criticisms of ineffective and inefficient allocation of resources. Criticisms range from development of amenities for students (Mendoza, 
2010; Woodhouse, 2015), the commodification of higher education (Lawson, Sanders \& Smith, 2015), ineffective space utilization (Janks, Lockhart \& Travis, 2012; NACUBO, 2016), and unchecked escalation costs for federal cost-share (U.S. GAO, 2016).

My research explored the need for organizational agility as examined through the imperatives brought by climate change, societal disparities, and increasingly limited resources; and it identified frameworks of systems thinking that offer applied solutions to real-world threats. These imperatives are the result of the interactions of many different systems of human activity (Crowley, 2000). At the heart of this dissertation is the recognition that the vast diversity and quantity of the world's knowledge experts are housed at or affiliated with institutions of higher education, and that there are significant hurdles to bringing together this wealth of intellect for the purpose of solving complex, intractable problems. Higher education institutions are hosts to the most diverse and comprehensive knowledge enterprise on the planet. However, it is difficult to extract and apply that wealth of intellect due to the organizational structures within higher education (Crow \& Dabars, 2015). Many of these hurdles lie within the academy itself, and in the organizational structures and processes of the knowledge and research enterprises (Becher \& Trowler, 2001; Biglan, 1973; OECD, 1972). This research examined the organizational structures of higher education institutions in the frame of a systems organization, an approach that favors integrated structures over siloes.

Several institutions in the United States have recognized that there is an opportunity for the vast knowledge enterprise to more comprehensively contribute to the complex challenges facing their communities, and the world at large, through intentional system reorganizations. 
Arizona State University, led by Michael Crow, is arguably one of the institutions at the forefront of reorganizing the higher education enterprise to effectively serve the needs of a world in peril (Crow \& Dabars, 2015). A systems approach is an application of systems thinking, "a way of looking at the world in which objects are interrelated with one another" (Whitchurch \& Constantine, 2009). The importance of garnering the knowledge of many disciplines and perspectives is imperative given that the problems are the result of highly complex systems interactions. Issues such as racism, climate change, poverty, public health, or loss of biodiversity cannot be solved by any one discipline, or even multiple disciplines operating within their own siloes. These types of complex issues have been coined wicked problems by Rittel and Webber since they have no easy, singular solution (Rittel \& Webber, 1973). These wicked problems are not the result of a singular enterprise, and they cannot be resolved with a singular solution.

The organizational structure at Arizona State University contradicts the very premises espoused by Crow and Dabars in that the institution's Facilities department and campus design processes operate in practice and function separate from the education and research enterprise. ASU is a leader in the organization of transdisciplinary knowledge through the Wrigley Institute for Sustainability, but even this renowned problem-solving institution does not leverage the expertise and knowledge to truly optimize their own learning environment. Institutions have subject matter knowledge in the academic enterprise and professional expertise and experience in the operational enterprise, yet the organizational systems of higher education preclude the integration necessary to transform campuses into learning environments that encompass wellbeing and equity as embodied values. In a systems approach, compartmentalizing any part of the system degrades the efficacy of the whole system. 


\section{Statement of the Problem}

"This sounds more learning based and the academic side of the house rather than the physical facilities side.”- Campus Planner, R1 Institution

The term campus has different colloquial expressions in practice. It can convey the entirety of an educational enterprise such as a university, or it can serve as an applied term representing the lands and physical assets of an enterprise such as a corporate campus, or a healthcare campus. In university settings the contextualized use in conversation is often a reflection of where the term is used within the organization. University faculty and staff often reference the sides of a house - meaning the academic side or the operational side. Within the university administrative structure, the campus (the grounds and buildings) requires administrative resourcing which is manifested as the functional responsibility of a department or division. For these Facilities departments, the use of the term "campus" denotes an area of control and responsibility that is exclusive to their domain, their side of the house. In the cultural lexicon of Facilities personnel, the "campus" is not the larger "college or university" enterprise, it is instead the millions of square feet, sidewalks, and utility infrastructure for which these staff are responsible. Facilities departments control and own the processes for design, construction and maintenance of the grounds and buildings, and to these staff the term campus means the physical environment; separate from, but supportive of, the academic and research enterprise (Wright \& Wilton, 2012).

This distinction is a crucial piece of information related to this research study. The studies of the cultures of higher education are largely devoid of an analysis of the impact and 
influence of the physical campuses and their Facilities organizations. Bergquist \& Pawlak (2008) noted that even the cross-pollination of ideas from corporate cultures were not well received in the cultures of the academy, whereas Facilities cultures are cross-cutting across every market sector. While the cultures of corporations, healthcare, government, and academia may be independently recognized since their purposes and practices are different, the Facilities organizations that exist in all of these entities have their own distinctive cultures irrespective of whether the campus is for corporate, academic, medical, government or military purposes. Facilities is a juggernaut of an enterprise with their own culture comprised of a distinct language, metrics, and professional designations. Facilities professionals move readily among these sectors because of the consistency and uniformity of the work and culture. This will be explored in more depth throughout this dissertation as a critical consideration of organizational systems, and how a campus is designed.

This dissertation examined how the application of a systems approach, integrating the knowledge enterprise of a university with the professional practitioners in a facilities organization, can create a learning environment that is healthy and equitable.

\section{Research Questions}

The research approach I took was to examine structures and the processes that connect them. Structures are the organizational hierarchies, processes are organizational networks of relationships (Lichtenstein, Uhl-Bien, Marion, Seers \& Orton, 2006), as well as embedded artifacts of cultures (Schein, 2017). Processes are able to be mapped throughout an organization and this allows for the identification of pathways, which can then be assessed to identify obstacles, areas of acceleration, dead-ends, redundant loops, and so forth (Clegg, 
Josserand, Mehra \& Pitsis, 2016). The research questions that guided this study are:

1. What organizational system barriers are there to a systems approach in campus design?

2. What organizational system enablers are there to a systems approach in campus design?

3. How can the effectiveness of a systems approach in campus design be measured?

4. How can success be defined - what are the performance indicators that demonstrate a successful campus design?

\section{Purpose and Significance of the Study}

A campuses' facilities and grounds have to serve many needs. They must be functional for the diverse user groups. Faculty, researchers, staff, students, alumni, business partners, corporate sponsors and community members comprise the majority stakeholder groups, and their interests are widely varied and often conflicting. Facilities and grounds also must be run efficiently and effectively to help address affordability and the proper stewardship of finances. The facilities and grounds also serve as recruitment tools, cultural centers, community services, and economic drivers; from principal investigator recruitment to indirect costs, from student mental health to cancer research, and from ballet performances to ice hockey tournaments, a university campus has to fulfill many roles. The campus environment contributes significantly to many outcomes, yet the asset value of these physical aspects of the campus are not truly accounted for beyond the standard metrics found on financial balance sheets.

From conception to demolition, a building vacillates between asset and liability, and is often simultaneously viewed as both. At what points in a building's life this vacillation occurs is highly variable. As a financial obligation, the depreciation is almost immediate, yet from a qualitative perspective the value is somewhat whimsical. Assessed in terms of alumni 
memories, a student's respite from homelessness, a gathering of friends, or a space where a life-saving procedure was developed, and these campus spaces become invaluable. A building, a tree, a bench along the walk - they all hold different types of values, and they can each illicit revenue, or become a money pit. Nothing that humans build lasts forever, and that is a liability. The health and safety implications increase as a building ages, as is evidenced by pieces of buildings falling off at the University of Wisconsin-Madison campus (Meyerhofer, 2021). Yet, the feelings, memories and epiphany moments may be more enduring and create a stronger allegiance than can ever be reflected on a general ledger. Ironically, that enduring allegiance is what the fundraising arms of institutions count on and benefit from. A money pit for the Facilities department may be critical to the fundraising arm, but those transactional relationships do not show up on the same ledger, or even in the same organizational structure since the philanthropic arms of universities are often separate from the institutions they serve. Their relationship, as demonstrated by processes, are not evident.

Higher education campuses often provide residential, classroom, research, recreation, dining, athletics, healthcare, animal care, and a myriad of other types of spaces. It is also imperative to consider that humans spend nearly $90 \%$ of their lives indoors (Klepeis et al., 2001). The implications for human and animal wellbeing within these facilities is a paramount consideration in facilities design and maintenance. There is also a need to ensure that spaces are optimized for use and can accommodate differing functions, schedules, and needs of the occupants. Given the intense financial, environmental, and human resources needed to build and maintain a campus, it is critical to get it done right the first time and to ensure that successful operations can be continued for the life of any given facility or landscape. 
Buildings and grounds require ongoing maintenance and a continuous infusion of labor and finances. In the course of its lifetime $15-25 \%$ of a building's costs are in the design and construction, and $75-85 \%$ of its costs are in the operation and maintenance (Darling, 2019; Fuller, 2016). This is the total cost of ownership of the building and grounds, and also includes the personnel costs in operation and maintenance. These are the budget numbers that show up on the Budget Office's ledgers and show Facilities departments as being cost centers (non-revenue generating departments). Optimizing the organizational system structures and processes through the inception and life of a building will reduce the total cost of ownership, maximize its use and effectiveness, and serve the institution in ways that provide additional value and benefit to the institution.

This research study analyzed existing facilities design processes at a doctoral institution with very high research activity and piloted five different models of an integrated design approach at different stages of the processes. Due to the recurring and repetitive nature of campus design, it was possible to run some pilots concurrently and others consecutively. One pilot study undertook an integrated stakeholder approach to classroom remodels. Another evaluated the addition of furniture into informal learning spaces (hallways). A third piloted a third-party architectural design tool on two capital projects: a renovation and addition at the School of Veterinary Medicine, and the replacement of a Natatorium/Gymnasium. The fourth study was an iteration of the third pilot and was applied to two more capital projects (a College of Engineering building and a Computer, Data and Information Sciences building). The fifth pilot study was the most granular of the process analyses. It analyzed the service level processes related to campus lighting and accommodation services. 
These pilot studies spanned internal (UW-Madison) and external (UW-System and State of Wisconsin) processes due to the capital and classroom design process extending throughout the vertical hierarchy of a state university system. These systems processes were selected for this study to analyze how the campus design and planning processes contribute to outcomes for the University of Wisconsin-Madison as identified by the State of Wisconsin (legislated oversight), Higher Learning Commission (the accrediting body), selected cognizant agencies for National Institutes of Health (57\% of UW-Madison's federal research dollars), and the Association for the Advancement of Sustainability in Higher Education (the national sustainability rating organization for higher education). Additional mapping of contributing processes for academic program planning, all-agency projects, landscape design, and utility master plans were analyzed against strategic planning processes for academics, research, and student life. The research identified where processes optimized or inhibited an integrated systems approach to the design, use, and operation of the campus environment.

\section{Limitations and Assumptions}

The study was limited to a public doctoral, very high research activity institution with medical and veterinary programs. The size and scale of these programs typically have unique facility and programmatic needs, additional compliance and legislative requirements, as well as more diverse fund sources including corporate partnerships and large donor support (Carlson, 2012). The complexity and critical nature of the built environment at this institution is compounded by high value (and sometimes controversial) research and healthcare operations. The unique needs of an R1 institution with medical and veterinary programs, mandate different levels of strategic planning and processes than are necessary in other types of institutions. The 
complexity implied a predisposition toward coordination and integration among many different departments, agencies, and offices.

A limitation is that data derived from Facilities organizations is quantitative and generally measures the performance of buildings as a reflection of the performance of the Facilities organization, but energy reduction metrics do not reflect how a building contributes to learning, research or occupant comfort. Facilities professional organizations and trade publications may have biases toward best practices, and do not generally expound upon the limitations and failures of their organizations in print or presentations. Public comments, meeting minutes and correspondence with Facilities administrators and staff were referenced to obtain qualitative data regarding organizational and process effectiveness.

This study included five applied research projects conducted at a doctoral institution with very high research activity (the University of Wisconsin-Madison). Four were aligned with institutional level planning (the capital planning group), and the other was a service level process analysis. Pre and post assessments of the applied integration projects were developed to ascertain levels of integration and how they contributed to the intended outcomes of the projects. Processes for these types of efforts are generally well documented due to compliance and financial reporting requirements. However, there are limitations to identifying which inputs may be missing from the processes. For example, the Americans With Disabilities Act legislates certain requirements in the built environment, but that does not mean that the accommodation services team is consulted as part of the building design process. Therefore, opportunities to incorporate inclusive or universal design concepts, or address physical and mental health outcomes, are often missed. Occupancy assessments and stakeholder 
conversations are necessary to find areas of conflict or dissatisfaction, and to identify additional services, costs or programs that have been developed to mitigate problems resulting from noninclusive processes (Tookaloo \& Smith, 2015).

The accommodation services process is an example of a process conflict which directly impacts learning, wellbeing, and equity. This study analyzed a process developed by the accommodation staff to address the increasing number of client accommodations needed for harsh lighting. Developing integrated processes was time-consuming, the accommodations staff did not even know where to begin to fix the process, and there were no connected work order systems or tracking mechanisms to provide data to quantify the issue, so the accommodation staff's recounting of their process and the impact to students was anecdotal. The other stakeholders quite literally had no idea that there was any kind of problem, nor that the institution was expending additional human and financial resources to address a rapidly burgeoning issue impacting learning, wellbeing, equity.

It was also important to identify existing stakeholders who may have undue weight in a process, thereby limiting the inclusion and input of other members of the university community. In essence, it was necessary to identify who was currently involved in the process, what level of influence they should have in the process, and who was not included but should have been. Integration has political implications for the various stakeholder groups, some of whom hold more power than others within the academic enterprise. The power differential is also at play within the vertical hierarchy, further limiting a clear delineation between the formal (command-control) and informal (adaptive) processes even for areas of the process that should clearly be within institutional control. 


\section{Definition of Terms}

The integrated approach to this study brought together disparate disciplines and professions which required a common understanding of shared language. The following definitions were helpful in ensuring effective communication between and among organizational cultures within higher education.

Adaptive Organizational Systems: The informal, relationship based, networks that exist among and between organizations. These are human-centered and fluid structures based on affinities, affection, and purpose.

Attribute: A physical manifestation of a design feature. For example, paint color, flooring and furniture are considered attributes of a space.

Campus: The physical and natural environment within the institutional boundary of a university or college.

Command-Control Systems: The traditional organizational structure of layers of authority and direct reporting lines.

Dependencies: A term used in project management to describe a sequence of events that require the completion of one before the next can begin.

Equity: Enabling the full range of human diversity without limitations or barriers that would preclude participation and opportunity.

Forensic Mapping: The retroactive analysis of a process to determine points of acceleration or deceleration to identify aspects of the process that contribute to success, failure, or stagnation. 
Learning: The process, over time, of acquiring new knowledge and understanding. In terms of this study, it includes the physiological processes associated with cognition and awareness.

Project Management: A role, that in the facilities and construction sectors, coordinates the logistics of a project from its start through its completion and turn-over to the owner.

R1 University: A doctoral granting university with very high research activity as designated by the Carnegie Classification System (Carnegie Classification System, n.d.).

School/College/Institute/Division (SCID): An organization within the University comprised of many academic or specialized departments and generally led by a dean or executive leader. For example, the School of Engineering or the College of Letters and Science are organizationally comprised of many disciplines. A Division of Student Affairs is similarly comprised of many specialized sub-units.

Scope: The boundaries or limitations of a project, generally clearly delineated at the project outset. For example, a parking deck project may not include landscaping, so if the client asked for trees, that would be out of the scope of the project and require a change order and additional commitment of resources by the client.

System: A set of related parts forming a holistic organization.

Transdisciplinary: A blending of academic disciplines, professional experience, and practice to structure knowledge to address an issue (Brown, Harris, Russell, 2010).

Wellbeing: Broadly defined in terms of this study, this encompasses both mental and physical wellbeing. 


\section{Organization of the Study: Conceptual Framework}

This research project examined systems approaches and crossed boundaries of disciplines, professions, and social communities, therefore a transdisciplinary approach was required to bridge the various paradigms. This research employed a framework established by Thomas Jahn to address social issues through a process that he termed the integrative mode of access (Jahn, 2008). This framework brings together separate knowledge into a real-world application through a process which assimilates the knowledge into an applied purpose. Jahn's framework was developed to address the need for disciplines with different methods to come together to solve problems.

While the integrative mode of access method is based off of earlier concepts such as Ackoff's approach to stakeholder consensus and idealized planning (Jahn, 2008, p 21), Jahn's framework considers external pressures to the ideal scenario - things such as finances, people, regulation, and other considerations that mess-up utopic plans. I once heard an architect lament that his building looked and performed flawlessly - until people were allowed in and ruined its performance. The integrative mode of access framework not only allows for the externalities, but it also incorporates them into the process so that they become intrinsic to the solution. It accounts for letting people into the building and accommodates what they do inside.

The challenges involved with varied disciplines, multiple methods and diverse cultures are not directly addressed in Jahn's framework, so additional guidance was provided through the adoption of methods developed by Bergmann, et al. (2012). For the purposes of this research study the frameworks for integration through models were used to adapt the integrative 
mode of access to the specific paradigms unique to this study. The integration through models framework has a specific model called integration through a system model which fit the need to map the processes within the organizational system and addressed the project in three phases: "Phase A: Project construction, Phase B: Project execution, Phase C: Project results/Bringing results to fruition" (Bergmann et al., 2012, p 102). An additional step in this applied research project was a review process, which has not been formally published, but was developed by Leith Sharp, the director and lead faculty of the Executive Education for Sustainability Leadership at Harvard's Center for Health and the Global Environment. Leith uses a process she has termed forensic mapping to review the progress of a project in terms of where it advances and where it is stalled, and she maps that progress against organizational systems so that it can be determined if the enabling/disabling points in the timeline are the result of a position, person, policy, or process. This review is critical to assessing the organizational relationships and the ability to take a systems approach in resolving issues.

With these frameworks in place, the research was conducted through a mixed methods approach. The qualitative methods included: reviews of project documents; published interviews; mapping, and analyzing the organizational processes; mapping, and analyzing the informal and formal organizational systems; and observation. Quantitative methods included analyzing financial, sustainability, accreditation and state performance funding data, and occupancy surveys. The prioritization models for the integrated design approaches used qualitative, quantitative, and a mixed method (pairwise comparison) measure. 


\section{CHAPTER II: REVIEW OF THE LITERATURE}

\section{Background}

A systems approach is the practice of identifying multiple problems and addressing them through a highly collaborative, integrated process (Brown, Harris \& Russell, 2010; Crow \& Dabars, 2015; Jackson, 1982; Senge, 1990). The premise of a systems approach, to tackle complex issues that have many inputs and outputs, has been identified by researchers on transdisciplinarity as a challenge in the academy (Brown, Harris \& Russell, 2010; Crow \& Dabars, 2015). It works in opposition to the traditional academic practice of identifying a single problem and addressing it through the lens of a single discipline or a single researcher (Brown, Harris \& Russell, 2010; Crow \& Dabars, 2015; Jackson, 1982; Senge, 1990). A review of the literature identifies that the fundamental issue with the application of a systems approach in a university is one of conflicting processes and cultures within the organization. This left me pondering how a systems approach could be applied to organizational structures and processes responsible for designing and building campuses that are purposed for learning and research, and how to identify the unique constructs and habits within departments as manifested practices and processes.

The structures of higher educational organizations have some similarities with corporate structures, and the models presented by various researchers and authors provide templates for how the structures affect people's behaviors and work outputs (Bergquist \& Pawlak, 2008; Bolman \& Deal, 2013; Schein, 2004). From hierarchical structures that can provide efficiencies yet lose communication capacity, to lateral systems that enable communication but lack direction and maneuverability; there are numerous examples of how 
certain frames work, and do not work, across diverse organizations. Higher education organizations are an amalgamation of vertical hierarchies and flat systems, with unique attributes of faculty autonomy and shared governance. Organizational relationships and structures vary across departments and disciplines, and this creates different organizational structures throughout the overarching institutional structure. Within a College or School the departments are fairly autonomous, and they can be dynamically different in how they are structured.

To provide some context, I will use a fictitious university (Green University) to explain the organizational structures of higher education. In this example, we have a student getting dual degrees in Computer Sciences and Theatre. These degrees are run by two departments (Computer Science and Theatre), and these departments fall into two different Schools/Colleges (School of Information Sciences and College of Fine Arts), which are just two of Green University's 20 Schools/Colleges which offer 250 degrees in total. The departmental cultures, curriculum, equipment and classroom needs vary greatly. However, the student's experience and the student's success are not solely impacted by curricular offerings. The student's physical health and mental wellbeing are critical considerations as well, and Green University has counseling centers, recreation facilities, multi-cultural centers, and dining centers to address these needs. Creating a systems approach for integrated facilities decisions across the hundreds of departments within an institution is a daunting endeavor. Yet there is substantial research to support the need for systems integration and identify the processes which have proven most successful in complex organizations. 
The structure of an organization impacts its ability to adapt and change in response to shifting internal and external forces (Bolman \& Deal, 2013). At an institutional level, legislators, students, parents, funding agencies and employers put pressure on higher educational organizations to be fluid, affordable and responsive. Individual academic departments and disciplines are not immune from external (and internal) judgements that threaten funding and societal acceptance. For example, the humanities have long been scrutinized for their relevance, as the support for the hard sciences and math has grown (Biglan, 1973). Yet the social sciences are regaining their importance in light of the persistence of social disparities laid bare by the global pandemic of 2020 (Nietzel, 2020; Ostherr, 2020).

Organizational structures that allow for innovation, communication, collaboration, and strong leadership are most adept at weathering a challenging organizational climate (Keeling, Underhile, \& Wall, 2007; Kezar \& Holcombe, 2017). While many of the structural theories present some type of vertical hierarchy, the organizational structures that resemble and incorporate a team concept provide better outcomes for personal fulfillment and increased engagement, while simultaneously meeting the organization's goals (Bolman \& Deal, 2013). Traditional hierarchies do not readily enable team or systems approaches without going through a command-control directive (formal orders such as setting up committees, task forces or working groups), however other types of networks exist informally within and among organizations. These informal networks are adaptive and responsive and built on relationships rather than hierarchical structure (personal conversation, L. Sharp, Harvard University). A successful organization takes advantage of the available talents and skillsets of individuals 
across the organization and enables movement between the command-control structure and the adaptive organizational structure, to achieve a larger purpose.

The focus of this research is the organizational structures related to campus design and planning, and the literature review reflects components of design and construction structures, and cultures of both higher education and the Facilities sectors. The professions in the engineering and construction field operate on a set of shared assumptions and processes encompassed by the practice of project management. Project management as a practice and discipline has been readily adopted and adapted by many sectors over the past fifty years, but the literature review will focus on how the practice of project management is applied in the design and construction of a Tier 1, residential, research campus (Kwak \& Anbari, 2009).

\section{Facilities and Learning: Relationship and Impact}

This dissertation is focused on the process of creating learning environments and is therefore premised on the assumption that there is a direct connection between the environment and learning, wellbeing, and equity. The literature supports this assumption. There is extensive research, particularly in K-12 schools, on the relationship between the environment and student achievement. A 2016 literature review by Bijaya Nepal identified over 700 primary documents that contribute to the research in this area. Indoor air quality, temperature, humidity, lighting, safety, cleanliness, and sound are commonly referenced attributes of the built environment that impact learning (Berglund, 2019; Cash \& Twiford, 2010; Earthman, 2002; Schneider, 2002). Of particular note are the repeated findings of a 5-17 percentile difference in student achievement depending on the quality of the buildings (Earthman, 2002). Students are also impacted by the experiences of their instructors, and it has been found that "poor school facilities negatively 
impact teacher effectiveness and performance" which also has a negative impact on student achievement (Earthman, 2002). Researchers have repeated similar studies in a variety of states, urban and rural settings, and have consistently replicated the results that show that the learning environment influences student outcomes (Cash, 1993; Earthman, 2002; Hines, 1996; Karna \& Julin, 2015; Kok, Mobach \& Omta, 2011).

The focus for much of the post-secondary environment is on student satisfaction and faculty recruitment as a function of adding value to the over-all experience of university (Crow \& Dabars, 2015; Karna \& Julin, 2015; Kok, Mobach \& Omta, 2011). In the disaggregated structures of a university, students assess spaces that they use, such as library spaces, housing, dining, athletic venues, etc. as separate and distinct experiences (Kärnä \& Julin, 2015). There are not standard user assessments of campus facilities in the aggregate, and typically each of the separate organizations (Athletics, Housing, Dining, etc.) have their own facilities budgets, facilities administration, and sometimes facilities staff. This is important to note because at a residential university learning occurs everywhere and most active student learning occurs outside the classroom (McLaughlin \& Faulkner, 2012). Therefore, the literature on learning environments and the attributes that are conducive to learning, wellbeing and equity are applicable throughout a university's campus. Due to the siloed nature of a university, the quality of those environments may fluctuate across spaces due to the autonomy of the divisions within a university's organizational structure.

\section{Attributes and Their Impact on Learning, Wellbeing, and Equity}

The study of how humans learn is a critical component of research in education (Sousa, 2011). The assessment of a particular space (e. g. a classroom, a lounge, a coffee house) to 
ascertain its impact on learning can be done by evaluating the attributes of the space against the known impacts to learning. For example, research on lighting has identified impacts to productivity, sleep, security, mood, and biological cycles - all of which can contribute to, or detract from, learning (Küller, et. al., 2006; TCP, n.d.; Winterbottom \& Wilkins, 2009). Using existing data on how various physical space attributes impact learning (e. g. the degrees Kelvin of light is not as important as the lumen levels for reading, but degrees Kelvin can be critical in a research laboratory), we can assess how a combination of those attributes can directly impact learning in a given space (TCP, n.d.).

Of particular interest to this study are the attributes that impact human learning, wellbeing, and equity. The research focuses on a few attributes that have a positive impact on all three design priorities; lighting, color, ventilation, sound, and exposure to nature (Holmes, 2018; Lee, Kwon \& Lim, 2016; Kurt \& Osueke, 2014; Methorst, et. al., 2021; Monaghan, 2019; Norris, et. al., 2008; TCP, n.d.; Winterbottom \& Wilkins, 2009). Building codes govern some of these features, such as ventilation, and the pandemic of 2020 prompted people to pay increased attention to air exchange rates. Other attributes, such as lighting, color and acoustics, are more complex and have variability on their impact on humans due to physiological differences in the population. For example, age is an important determinant on the impact of light and sound. Younger populations can hear more frequencies and need less light for reading, whereas older populations may require environments that directly conflict with the environments that benefit younger people's ability to learn and feel comfortable in a space. Achieving equity in the university environment is functionally impossible. Striving for equity and the removal of barriers is the priority focus for inclusive design (Holmes, 2018). 


\section{Code Compliance, Social, Physiological and Health Imperatives in Campus Design}

University research campuses are comprised of many different types of buildings, rarely designed to serve a single purpose. Throughout their lives, campus buildings routinely serve as dining halls and residential spaces, bio-safety laboratories, vivaria, classrooms, clinics, offices, and libraries. The people, plants and animals that use these spaces over the course of decades are even more varied than the facilities that serve them, yet the building and construction process has yet to mature to the point of being able to accommodate the complex, and sometimes conflicting, needs of occupants. Humans have long adapted their environments to suit evolving needs, and the building sector has increasingly created uniformity in design and function that spans climate, terrain, and culture. This has advantages and disadvantages. For example, uniformity in safety, sanitation, and the licensing of trades and professional personnel provides a high degree of confidence by building occupants and owners that a facility will have appropriate egress in the event of a fire, or that a plumber is qualified to work on both potable water and sewerage systems. But uniformity also has drawbacks, and these are often glaringly apparent when buildings are occupied by highly diverse campus populations.

Uniform building codes mandate certain features and attributes for the built environment that address fire safety, structural collapse, sanitation and similar health and safety considerations (Leon \& Rossberg, 2012). Building codes are also established in response to societal pressures and public policy such as the adoption of codes for energy efficiency prompted by the OPEC oil embargo in 1973 (Hunn, 2010), and accessibility prompted by the Americans With Disabilities Act (Jones, 1991). The laws of physics remain constant with regard to building materials and construction, thereby allowing a degree of consistency in structural components of the codes. 
However, climate change and the evolving laws and cultures of societies are more challenging to translate as manifestations into the built environment. Even the aspects of building codes related to structural components, concrete for example, are now having additional metrics tacked onto their use to track embodied carbon and the social costs of carbon (Stadel, et. al., 2011). The building sector has subsequently evolved green building rating systems such as LEED (Leadership in Efficiency and Energy Design), WELL (International WELL Building Institute, and Living Building (International Living Future Institute). These building rating systems provide sets of criteria to achieve particular objectives - such as wellbeing, public health, and climate change resilience - that go beyond code and are more responsive to societal pressures and human behavior than federal or state mandates (Zhang et al., 2019).

There is limited research on the institutional reasoning behind adoption, or lack of adoption, of these green building rating systems in higher education. Research that has been conducted involves case studies of institutions and surveys of students which have shown similar organizational barriers in the institutional decision and financing processes. One of the most prominent findings in the research is the nexus of control of facilities departments in the campus design and construction decision making process, and the focus on energy efficiency as a design priority over other attributes that are prominent in green building rating systems. The organizational structures in higher education are more robustly researched and shed additional light on the decision-making processes related to campus design (Golbazi, El Danaf, \& Aktas, 2020; Kim, Sunitiyoso, \& Medal, 2019; Richardson \& Lynes, 2007; Wright \& Wilton, 2012). 


\section{Applied Systems Thinking}

The literature on systems approaches encompasses expertise from across the academy, incorporating knowledge sets from many disciplines. Expertise on organizational systems can be found in anthropology as easily as it can be found in the business school or biology, and a variety of perspectives may well be needed depending on the problem being examined. Terms such as multidisciplinary, interdisciplinary, and transdisciplinary are commonly used to describe levels of integration of different types of systems and knowledge. "Interdisciplinarity is not a panacea for change in the universities, but it is a vantage point from which a good deal of critical and healthy reflection on the inner workings of the university can be stimulated." (Organisation for Economic Cooperation and Development, 1970, preface). In September of 1970, the University of Nice (France) hosted a week-long seminar titled "Interdisciplinarity: Problems of Teaching and Research in Universities." Organized by the Centre for Educational Research and Innovation (CERI), the seminar is cited as the first of its kind to bring together scholars from around the world and across disciplines to examine how collaboration "unifies knowledge" and what the impact of such an endeavor could be. (Organisation for Economic Cooperation and Development, 1970). The premise of the seminar, as championed by its director at the time, J. R. Gass (1970), was not to limit the disciplines, but to help them contextualize their work within academia and within society.

The literature describes the brief early years of the 1970's as a transformative time with the beginnings of discourse on a new systems approach to the academic and scientific enterprise, but then issues in the world, largely OPEC and the oil shortage, diverted funds, resources and attention (Bernstein, 2015). Nearly two decades later, the global community 
began to discuss issues of Earth's sustainability and spawned a new era of discussion on transdisciplinarity. Precipitated by the Brundtland Report in 1987, the United Nations developed the Conference on Environment and Development in 1989 which was followed by the United Nations Earth Summit in Rio De Janeiro in 1992 (Bernstein, 2015). The need to address non-sustainable consumption, and its effects on humans and the environment, required knowledge and expertise from across the disciplines, and it required a new and collaborative approach. Transdisciplinarity was seen as a solution to the intensely complex systems issues that were challenging the globe, and now a global pandemic is further highlighting the interconnectivity of these complex systems.

The wicked problems of poverty, pollution, declining human health, loss of biodiversity, income disparity and climate change, to name just a few, need the full capacity of brilliant minds from all corners of the academy (Rittel \& Webber, 1973). The theories behind transdisciplinarity as a way to address wicked problems are derived from the study of knowledge within and among the disciplines. In practice, the scholarship of knowledge is a transdisciplinary approach (Bernstein, 2015). In 1962, Professor Fritz Machlup proposed means by which to calculate the economic impact of knowledge (Machlup, 1962). He examined knowledge enterprises, education being one of the largest, but he also examined corporate research and development, media, information services, and information machines which we now refer to as computers. In light of his work and the framing of his approach the impacts and transportation of knowledge can be examined in a tangible way.

Quantifying knowledge, particularly in terms of monetary metrics, enables knowledge to be added to the global economic ledger. For example, the global balance sheet now 
demonstrates how lack of access to education impacts poverty, health, the environment, and persistent social disparities as well as when and where investment in education can mitigate a negative balance. Knowledge as a transferable commodity shows up in every economic transaction and in every academic discipline. Transdisciplinarity is not just an academic method of approaching a problem, it is a framework for tackling the most challenging of the world's problems in a transformative, system-wide, approach. How that approach is enabled in higher education campuses is the focus of this dissertation.

The organization of administrative systems in higher education have been developed around the academic disciplines. Functional services such as admissions, facilities operations, budgeting, and human resources encompass the entire enterprise, however academic freedom is a unique organizational attribute applied only to a sub-set of employees (faculty) in the higher education systems in the United States (Meyer, 1967). While the Brundtland Commission laid the groundwork for a methodological approach to transdisciplinarity through the global adoption of the United Nation's Sustainable Development Goals (United Nations, 2015), the administrative and organizational systems within higher education do not readily accommodate the adoption of a similar framework. Authors exploring transdisciplinarity focus on the disciplines and the silos embedded in higher education organizational structures (Bernstein, 2015; Evans, 2015), but there is limited exploration of this topic from purely administrative process perspective (personal conversation, J. Klippel, University of Wisconsin-Madison).

Processes such as how funding is distributed for enrollment, class assignment, overhead for grants (indirect cost calculations), laboratory and classroom scheduling, are just a few of the administrative processes that were not designed for transdisciplinarity. The creativity 
needed to navigate these processes proves to be exceptionally difficult for university budget officers, admissions specialists, and development officers (personal conversation, J. Klippel, University of Wisconsin-Madison). This dissertation will diverge from previous research on the topic in order to explore how higher education organizations are encumbered by past practice, policy, and legislation in their efforts to advance a systems approach in campus planning and design.

\section{Organization of Higher Education}

The determinants of which knowledge areas and practitioners are grouped together has been examined in terms of paradigms, subject areas, and communities of practice. In examining how these "cognitive communities" (Brown, Harris \& Russell, 2010, p. 40) are assembled, Brown, Harris, and Russell (2010) summarized several researchers' standards as “a set of cognitive goals and methodological procedures; tolerable error limits; and substantive assumptions about the domains under investigation" (p. 40). What was also noted is that the sets of assumptions upon which each community is built are based upon prior sets of assumptions, and those in turn were based on earlier assumptions. The analogy of a foundation of knowledge created by building blocks is a fairly literal comparison. The building blocks that form the organization of knowledge also provide the foundation for the organization of institutions of knowledge.

Universities and colleges serve critical functions in the knowledge enterprise. They serve as disseminators as well as creators and curators of knowledge. This is often manifested in institutions as two distinct areas of knowledge organization: the academic enterprise and the research enterprise. While there is a great deal of overlap between them, many institutions 
have developed administrative departments and processes to serve and support these as distinctive functions. One of the administrative needs is data reporting for various regulatory, funding, and reputational ranking purposes. These data sets are also used nationally to create a taxonomy of institutions. One of these classification systems is the Carnegie Classification of Institutions of Higher Education. Established in 1970, it rates institutions on factors such as degree levels offered and program focus. The Carnegie system differentiates institutions by research intensity and number of students in undergraduate and graduate programs. While there are dozens of other institutional rating systems, the Carnegie Classifications are often used to note the capacity of the research and academic enterprises of institutions. Using this system, this research will examine a select R1 institution, designated as having very high research activity.

Institutional and organizational structures within American higher education have evolved over the past four centuries to accommodate both expanding knowledge and a growing number of disciplines, although the evolution has not always been in parallel. At some point in the early twentieth century the development of advanced post-secondary education, particularly in the area of the professions, began to take hold (Thelin, 2011). The creation of masters and doctoral programs to denote an exceptional level of expertise also led to a robust build-up of capable scientists who were ripe for the federal government to leverage as the United States entered World War II. In 1945, a report written by Vannevar Bush promoted the use of science as a means to maintain forward momentum for the United States (Thelin, 2011). This led to the creation of programs that funded university scientists to develop solutions for government projects. This in turn led to universities ramping up their research 
programs. The universities with very high research activity, categorized as R1 institutions in the Carnegie Classification System, generally receive a significant amount of federal research dollars. In 2018, 642 universities received nearly $\$ 42$ billion in federal research dollars, with the top 15 recipients (all classified as R1) awarded over $28 \%$ of the total (National Center for Science and Education Statistics, 2019, table 23).

The research enterprise is an integral component of knowledge development and it can require intensive resourcing, particularly in the science and engineering disciplines. Of the $\$ 80$ billion in research funding awarded to universities from all fund sources in 2018 , only $\$ 4.6$ billion was allocated outside of science and engineering (National Center for Science and Education Statistics, 2018, table 3). While organizational systems and functions vary across the classifications of institutions, research enterprises require dedicated administrative systems. Research occurs in industry as well, but higher education is a unique system that allows for an interplay between industry, government, curriculum, and the community. While industry may offer limited internships or post-docs, higher education has leveraged the research enterprise to expand learning opportunities to undergraduates in an integrated approach that complements the dissemination and growth of knowledge.

In Thelin's work, A History of American Higher Education (2011), he examines the foundation and development of post-secondary education in the United States. The entirety of U.S. institutional history has been a series of transformations in response to a variety of pressures. Higher education is not static. As the embodiment of an ever-growing body of knowledge, institutions are by their very nature agents of change. As Thelin notes, higher education is repeatedly subject to internal and external tensions which transform, albeit slowly, 
the educational system. Politics, ethics, culture, religion, finances, and a nearly exhaustive list of other factors are at all play, and the products of universities (in the form of research and graduates) not only inform those systems, but they are also influenced and changed by them as well. Clark Kerr, a former Chancellor at the University of California-Berkeley, and President of the University of California noted that tension and conflict are inherent at universities. In his work, originally published in 1963, Kerr characterizes institutions of higher education as "Multiversities" (Kerr, 2001 ed., Chapter 1), stating that "The University is so many different things to so many different people that it must, of necessity, be partially at war with itself' (p. 7).

R1 institutions, as purveyors of knowledge and powerhouses of research - some of which is controversial, may prompt conflict but they also examine conflict through research on the complexity of organizational systems, learning organizations, and chaos theory. What is of note in the literature on higher education organizations is the emphasis on the history and culture of the academy, diversity, knowledge, research, and student life. What I have found to be lacking is the research on the machinations and functioning of the university. The internal mechanisms of the system are discussed - financial aid, student health services, academic departments, shared governance, grant funding, advancement - there are books and studies on each area of higher education. These pieces are examined as if the multiversity is a plug-andplay entity, with components that can be added or subtracted at will. What I have struggled to find is the research on how they work together as a system. The extant research examines pieces of the components, and the curriculum also teaches the functions as compartmentalized pieces, but this also misses the systems approach. 
Institutions are viewed as singular entities but operate as decentralized individual agents. The segmented operationalization of higher educational institutions has imposed some limitations on the knowledge enterprise, and it has also exacerbated the conflicts inherent in universities (Becher \& Trowler, 2001; Bergquist \& Pawlak, 2008). These conflicts manifest at an individual level, as exemplified at Indiana University at Bloomington with Professor Eric Rasmusen's espousing of his personal views in hate-filled speeches. These types of actions gained national attention for their pervasive bigotry and misogynism (Pettit, 2019). The actions of an individual required the institution to develop processes by which other individuals are not harmed as a result of the professor's personal beliefs (Pettit, 2019). Similarly, but at a larger scale, applied research within a discipline can promulgate solutions which, when applied in the real world, create additional harm in other sectors.

A simple example of an unintended consequence, and an example of the longitudinal amplification of impact, is the chemical development of plastics. One of the many things that plastics are made into is the tubing used in the medical care of infants in neonatal intensive care units. Decades after its discovery and adopted uses, it was discovered to leach phthalates into tiny babies, further compromising their already vulnerable systems (Demirel, et al., 2016). Physicians and nurses worked to solve the problem of sick babies who subsequently manifested developmental disabilities as they aged. Families have been forced to live with the debilitating consequences and expenses, the educational system had to develop the knowledge and resources to enable learning and create a suitable environment for the children, and social service agencies must serve the needs of these individuals and their caregivers for the duration of their lives. 
There are two aspects of this real-world example that can be addressed through a systems approach, the aspects of connections and time. The manner in which institutions and knowledge are organized promotes "specialization and places a premium on expertise" which creates a further "division of cognitive labor" (Williams, 2001, p. 9). Specialized knowledge also moves along a time continuum, building upon what has been previously discovered, but not at the same rate of speed or chronological order. Learning is sometimes a process, sometimes an event, and sometimes an accident. One of the many challenges of a compartmentalized organization of knowledge is that it leads to unintended, but potentially avoidable, consequences. These conflicts may not be the result of a lack of knowledge (although in some instances that is the case), but as a result of the artificial boundaries that limit collaboration. Knowledge specialization has allowed for truly amazing and invaluable advances in our knowledge base, as well as spawned new disciplines and methodologies. When Rittel and Weber (1973) coined the term wicked problem they were characterizing the effects of many disparately implemented solutions converging and acting on the real world in ways that were never intended (Brown, Harris \& Russell, 2010). Integration of knowledge is a method that may enable the best application of what we know to avoid unintended and harmful consequences.

\section{Evolving Conversations in Higher Education Systems: Transdisciplinary Practices}

Much of the discussion about knowledge begins with the concept of curiosity, which leads to inquiry, and then advances through the processes of discovery. While a young child may discover through play or learn through observation, university researchers follow a prescribed process which may involve discipline specific standards, legislated regulations, and institutional policies. As we age and matriculate through school we transition through the 
accepted forms of discovery and the manner in which we pursue our curiosities becomes more rigid. A kindergarten classroom is filled with color, art, spaces, and materials that encourage creativity while playing and learning together. A college classroom is likely to have fixed seating, neutral colors, no art, no opportunity to play, and limited opportunity to learn together. The discussions revolving around transdisciplinary learning and research are in some ways revisiting early childhood education in which we learned how to learn. The language used in the research reflects that. Words such as creative, imaginative, innovative, open, and collective are recurrent in the literature on transdisciplinarity (Brown, Harris \& Russell, 2010; Crow \& Dabars, 2015).

What is argued for in the work on transdisciplinarity is a new approach, a method to bring comprehensive knowledge to bear on societal problems (Bergmann, et al., 2012, Crow \& Dabars, 2015; Brown, Harris \& Russell, 2010).

A societal problem, the solution to which requires a scientific contribution, is rarely susceptible to specialized handling. The specialized knowledge of an individual scientific field can usually deal only with certain aspects of the problem. This suggests a key task for researchers - bringing together these disparate aspects of the problem. (Bergmann, et al., 2012, p. 23).

The recommendations for undertaking transdisciplinary research focus on a number of areas related to the disciplines, institutional organizations, and systems approaches in order to bridge the methodological, cultural and language differences between the disciplines. As noted in the observation by Bergmann, et al. (2012), specialized knowledge cannot tackle the complexities of wicked problems, but what is not 
explicitly stated is the premise that solutions may require non-scientists as well as scientists. This involves community members, artists, politicians, and other stakeholders. Transdisciplinary research requires methods and processes that can inform, and be informed by, very different knowledge sets and ways of knowing (Bergmann, et al., 2012, Brown, Harris \& Russell, 2010; Crow \& Dabars, 2015; Wenger, McDermott \& Snyder, 2002).

The challenges of an integrated approach are reflected in the disparate organization of knowledge and institutions of higher education. Processes and cultural norms provide comfort, security and familiarity which are critical components of the human need for survival. Changing these norms causes dissonance and discomfort, hence there is significant resistance to change these structures and cultures (Bertalannfy,1972; Habermas, 1972; Schein, 2017). In juxtaposition to the way that change is viewed as a threat to survival, a threat to survival is also a motivation to change (Brown, Harris \& Russell, 2010; Crow \& Dabars, 2015; Schein, 2017). As was noted earlier in the essay, tension and pressure on universities has prompted some significant changes. Crow and Dabars (2015) proposed a model of a reformative, integrated systems approach to counter the recalcitrance of higher education to organizational change. Ironically, studying a systems approach is something that institutions do well, but they appear to be lousy practitioners. Kerr reflected that he thought of a university as "a series of individual faculty entrepreneurs held together by a common grievance over parking” (Kerr, 2001 ed., p. 15).

\section{Summary of Scholarship on the Knowledge Enterprise and Transdisciplinarity}

A systems approach to creating environments and cultures in which transdisciplinarity becomes an accepted practice requires, not surprisingly, a 
transdisciplinary approach. The literature on a systems approach in higher education encompasses expertise from across the academy, and as was discussed, knowledge sets are used in different ways by different disciplines. Expertise on organizational systems can be found in agriculture as easily as it can be found in the business school, and an adaptable methodology may well be needed depending on the problem being examined. Established process analysis and business process improvement methodologies identified in the literature will be applied to this research project (Biazzo, 2002; Povey, 1998).

What became apparent to me while reviewing the core scholarship on this topic is that the knowledge exists, what is missing is a process. Ironically, what I also discovered is that when processes are created, especially when they are technology based, they are very difficult to change and eventually become an embedded part of the culture. This limits the flexibility and adaptability needed to address wicked problems and university processes. Figuring out how higher education can optimize its campus environments to contribute intentionally to its mission is as much of a wicked problem as how to enable the knowledge enterprise within higher education to address wicked problems elsewhere in the world. 


\section{CHAPTER III: METHODS OF ANALYZING ORGANIZATIONAL PROCESSES}

\section{The Study Proposal}

This is an applied research project intended to incorporate the existing research findings on environmental attributes that contribute to learning, wellbeing, and equity into the campus design process. The research objectives are to analyze processes, identify optimal points of integration, apply the change, and evaluate for effectiveness. The methods are derived from the application of organizational change management theories to the campus design process. A unique challenge of this study are the organizational systems in which this study is taking place. These organizational change methods are being applied in a higher education institution which is embedded in a deeply bureaucratic vertical organizational hierarchy comprised of three tiers of control. The selected institution is the state's flagship institution and has had a tumultuous decade of the state legislature reducing funding, attempting to strip tenure, and removing bargaining rights of some of the work force. Modifications and adaptations of the methods were necessary to accommodate the dynamic nature of working in an active institutional and political environment. The intended outcome is to optimize the campus environment to purposefully contribute to the mission of the university by meeting the needs of multiple imperatives: learning, wellbeing, and equity. Specific to this study is integrating these priority imperatives into the campus design process to achieve an intentional outcome, an environment intentionally designed for human learning.

\section{Statement of Positionality}

My interest in this topic is deeply intertwined with sustainability, my profession. Sustainability is an approach to systems complexity that strives for long-term positive 
outcomes in the areas of environmental health, human society, and economic vitality (United Nations, 1987). It has been my experience and observation that the human-centric focus of traditional sustainability thought leadership compromises the very premise of a systems approach, so I have adopted a slightly different schema in my professional work. Traditional sustainability focuses on human outcomes which skews the entire environmental system to benefit humans, an approach which is antagonistic to systems thinking. It is also a distinctly Western or European construct (Said, 1978). Whereas, approaching all biological entities as imperative to the health and longevity of systems incorporates non-Western cultural values, provides a more comprehensive framework for equity, validates the importance of diversity, and honors the core principle of systems complexity - interdependency.

The complex systems approach that I am using to frame this dissertation highlights two imperatives, adaptability and diversity. These terms have varied applications in higher education throughout the academic disciplines, and in the administration of institutions. In the biological sciences these terms are used to describe the resilience of organisms, species, and eco-systems. In higher education they are variously used to describe institutional features such as the ability to adapt to political and economic pressures, and the ethnic or racial diversity of the faculty or student body. In this dissertation I will use a living framework, such as those adopted in the biological sciences, to apply complex organizational systems theory to the operation and administration of higher education research institutions. This application acknowledges that organizations are similar to organisms in that they are not static but are responsive to their environment. The terms adaptability and diversity in the context of this study refer to an 
institution's ability to change processes and to integrate a wide variety of expertise and perspectives into those processes.

I have worked in higher education for nearly three decades, and I have a particularly unique niche having worked across the administrative and academic spectrums with a significant amount of experience with divisions of Facilities departments. This doctoral program has also added to my knowledge and understanding of higher education administration. The area in which I have the most exposure and experience is finance and facilities, and this has provided me a lens with which to examine how institutions are organized, and how critical diversity and adaptability are to the campus environment. It is through this lens that I will examine, and apply, a complex systems framework to a researchintensive institution, using processes related to the built environment as the nexus.

This dissertation is in itself an applied, longitudinal research project. My role at the university is that of a change agent. This allows me latitude to disrupt the status quo and provides the support to implement these theoretical frameworks with the intention of achieving targeted and long-term outcomes for the institution. That may seem to imply a bias towards having to achieve positive outcomes. However, given the organic nature and temporal aspects of changing the campus environment, the indicators of success for this dissertation are actually the mapping and integration of processes among divisions and departments. Assessment of the effectiveness of those changes will not be immediately evident. Implementing processes that incorporate feed-back loops, assessments, and incremental targets are expectations of my position and will aid in this research project. 
My experience working in facilities organizations has shown that there is a substantive disconnect between these operations and the mission of the university. I am not demeaning the capabilities of Facilities administrators and professionals; they are really good at their jobs and often hold degrees in Mechanical Engineering or Architecture. The disconnect is in the understanding and knowledge of how influential the built environment is to the success of the academic and research mission of the institution. Facilities personnel think, and their performance is measured, in terms of the performance of a building and the aesthetics of the grounds. Metrics such as deferred maintenance, square footage, energy intensity, emergency egress, and the efficiency of mechanical systems are their performance indicators. The campus buildings and grounds are viewed as structural assets that serve and support the institutional mission, and while that mission is touted by the leadership, the relationships are couched in terms of services, not contributions (Amaratunga \& Baldry, 2000). In my personal interactions, faculty often view the purview of facilities organizations as temperature control, custodial and maintenance, garbage collectors and lawn care staff, and sometimes as obstacles. Students are generally not even aware that facilities organizations exist. The educational and research missions are seen as something that facilities personnel support, but as outsiders. I am proposing that through a systems approach, the buildings and grounds can contribute tangibly to the mission. That the built environment is critical to a sense of belonging and equity, that the campus contributes to the wellbeing and mental health of students, faculty, and staff, and that the buildings and grounds are assets that have measurable co-benefits as positive attributes with financial implications for tuition, fees, indirect costs, auxiliaries, grant, and gift funding. 


\section{Research Approach}

The research on learning is extensive and is often conducted by university researchers. This may seem ironic since the application of the findings of the research are not well incorporated into the facilities design practices within higher education - universities do not necessarily practice what they teach. However, it is also an indication of the significant siloes that exist at universities. in their analysis of the higher education organizations, Engaging the Six Cultures of the Academy, Bergquist and Pawlak (2008) lump facilities departments into the managerial culture. That lumping could be construed as an acknowledgement that the Academe is dismissive of the impact of the environment on their effort. I have been a student at three universities and a community college, and I can recount countless examples of how disruptive the environment has been to my own learning, as well as that of my classmates. And the faculty and instructors expressed equal frustration with their ability to teach and engage with students in those spaces. Students and faculty are the stakeholders most impacted by the learning environment, and yet they are not included in the process of designing these environments.

In the education sector as a whole, students are not included in the design process. Instructors however, particularly in K12, are given great latitude in setting up and decorating their classrooms. A simple google search of a kindergarten classroom will pull up images of collaborative spaces, color, art, soft chairs, ample table space, and windows through which playgrounds can be seen. Even my daughter's high school classrooms are decorated by the teachers - her favorite is plastered with comic books and animation characters, the overhead lights are covered with leafy shade covers and floor lamps are dispersed around bean bag chairs and movable furniture. They are spaces that inspire and allow for different methods of teaching 
and learning, and they are the products of the teachers' design. Institutional spaces are generally devoid of color, have fixed seating or tablet armchairs with surface spaces that are too small for anything but a notebook, bad lighting, and poor acoustics. The pedagogical methods are often driven by the classroom set-up. These spaces are not designed for, nor with, student or instructor input. These spaces are designed for ease of maintenance and cost effectiveness and are based on campus standards that also do not include faculty or student input.

University facilities' design and maintenance processes have a singular focus on a particular space type as the place where learning occurs, namely classrooms. Yet, on an average residential campus, students spend less than 13 hours each week in a classroom. Institutions have not yet synthesized the research on how the environment impacts learning, wellbeing and equity into their processes related to the built and natural environment. The value of spaces throughout a campus are generally derived from construction and maintenance costs, and there is a strong financial and design focus on classrooms and laboratory spaces. A classroom space is often presented in terms of dollars spent per square foot, technology capabilities and student capacity, whereas natural spaces such as nature preserves are often listed as a cost center and a real estate asset. Yet if the value of spaces were framed in terms of learning, wellbeing, and equity, it is likely that the nature preserve would be some of the most valuable square footage on campus. This study applied the premise that the entire campus is a learning environment, addressed imperatives of learning, wellbeing, and equity in the design priorities, and incorporated metrics for these imperatives to all parts of a university campus. 


\section{Research Questions}

This applied study sought to transform the process of campus design and construction through a systems approach, to achieve a campus environment that intentionally and directly contributes to learning, wellbeing, and equity. To transform the standard and accepted campus design processes I focused on the following research questions:

1. What organizational barriers are there to a systems approach in campus design?

2. What organizational enablers are there to a systems approach in campus design?

3. How can the effectiveness of a systems approach in campus design be measured?

4. How can success be defined - what are the performance indicators that demonstrate a successful campus design?

\section{Applied Research Methodology}

To answer the research questions, a mixed methods approach was used to analyze the multiple processes and organizational relationships involved in the campus design process. The process of campus design is perpetual. This results in a great number of people interacting with, and influencing, the design over extended periods of time. The ability to analyze both the organizational process, and how people intersect with the process, required a dynamic approach (Maxwell \& Loomis, 2003). A dynamic approach is an interactive, systems-based approach. This suited the objectives of examining organizational systems of campus design that follow a sequential process of concurrent, as well as consecutive, interactions with many people amid simultaneous projects, each at different stages in the process. 
This dissertation is a pragmatic, applied research project and the methodology is outlined in this chapter. Due to the prolonged time frame from a project's conceptualization to its completion, it was necessary to review multiple data sources to gather information related to the project processes. The methodology was as follows:

1. Identify or develop process maps of existing capital planning processes at the University of Wisconsin-Madison.

2. Identify the attributes of the built environment that have been shown to have a positive correlation with student learning, wellbeing, and equity.

3. Examine processes at other R1 institutions that are rated/ranked higher than the University of Wisconsin-Madison in national rankings to identify potential best practices and models for integrating the identified attributes.

4. Develop and implement an integration process into the existing University of Wisconsin-Madison capital planning efforts.

5. Assess, modify, and reapply the integration process for the next rounds of capital planning.

The first step in the process was to complete the foundational work on process mapping to ascertain a baseline assessment. It was necessary to determine a benchmark to effectively implement and measure progress toward the study's objectives. This initial mapping exercise had a very important temporal aspect that was critical in identifying where and when in the process to interject the new design concepts. This provided a starting point, A, and an end point, $\mathrm{B}$, which then allowed for the exploration of change management options to navigate from A to B. Of particular importance was to incorporate strategies that addressed the unique 
culture of the academy and facilities department, as well as the antagonistic political environment in Wisconsin. These were critical differentiators in the analysis and planning.

The data sources are all public documents in the forms of meeting minutes, meeting recordings, project documents including e-mail correspondence, legislative actions, miscellaneous public reports from various agencies, and similar artifacts. This information was catalogued into process maps and analyzed using the Adaptive Operating System (Sharp, 2012), also known as an Organizational Network Analysis (McDowell, Horn, Witkowski, 2016). For the purposes of this study, I combined the Adaptive Operating System and the Organizational Network Analysis, to refer to the extensive informal networks of relationships among the entire vertical hierarchy of the University of Wisconsin-System as the adaptive organizational systems. The formal organizational hierarchies are referred to as the commandcontrol systems. The findings were then integrated and applied on two capital projects, and then assessed, refined, and integrated a second time on two more projects. Because this was an applied study, discoveries were made along the way which resulted in two additional studies that approached the integration process at lower points in the organizational hierarchy.

\section{The Researcher}

I am the primary researcher on this project and have worked in higher education facilities organizations for over 25 years, with the last 15 years serving in the emerging profession of sustainability. The role of a higher education sustainability professional is to apply systems approaches to optimize institutional processes in a manner that bridges the various divisions and disciplines of a university. My professional role and academic interests converged when I took 
an inaugural administrative sustainability position at an R1 institution and was tasked with transforming the culture of the university and addressing the sustainability deficiencies.

Given the nature of transforming a process from one that relies heavily on quantitative measures for performance indicators, to a process that incorporates subjective and social constructs, it was necessary to conduct a mixed-methods study. This is an applied study engaging in a live process within a vertical hierarchy and conducted from the position of an administrative role in a flat, and highly compartmentalized organization. Creswell notes that there are many classifications of mixed methods, and that there is an "evolving nature" to mixed methods research (Creswell \& Creswell, 2017). Although I started with a plan, adjustments had to be made along the way to deal with the realities of working in a live, complex organizational environment.

Because this was an active study to transform a process, the methodology I used had to be adaptive to move from the established process to the new process. The established methods and processes within the facilities' sphere of campus planning, and the unique organizational structures within higher education, provided a limiting framework for analysis and action. While I ultimately used the business process mapping method, it was not the first analytical method that I tried. I struggled to find an established methodology within the literature that fit well with this project, and as a result there was trial and error testing of methodologies. This experimental process became part of this applied research study. In this chapter I will use a chronological accounting of the process, review the successful and not-so-successful methods that were employed, and share the tools that were developed and applied. 
As an active participant in this research study, it is important to note that I have a bias toward developing a working solution. That being said, the current political direction of the State of Wisconsin after the 2018 and 2020 elections leans toward sustainability solutions, which also drove positive synergies and resources to this particular applied research endeavor. It is also important to note that this is an effort established to accomplish near, mid and long-term objectives, and is well situated to be a longitudinal study in organizational change management. The reporting in this dissertation is directly related to the processes involved in campus design and project development, and does not include measuring the outcomes that may result from changes to the project processes

\section{The Applied Pilot Studies}

Process mapping is a method used to graphically represent a flow of work and organizational relationships (Damelio, 1996). It is a useful analytical tool in any sector, and offers the analyst the ability to examine workflows, knowledge flows and formal (as well as informal) relationship (Wang, Zhao \& Zhang, 2009). It is particularly well suited to this research endeavor due to the complexities of examining the professional practice of campus planning and construction while simultaneously integrating the research on learning, wellbeing, and equity into those practices (Anjard, 1998). There are existing, and well-established processes and timelines for campus design and construction, and in those existing processes there is limited opportunity to introduce new methods or models.

For example, the University of Wisconsin-Madison has a ten-year cycle for campus master planning, therefore interjecting a new priority of wellbeing, manifested through increased natural areas in the master plan, can only formally be done once each decade with the current 
system. There are informal methods available to influence individual projects, but the overarching plan is a highly structured process with many approving bodies both internal and external to the institution. This decade long limitation required that the process change effort be focused on individual projects as they became available in the campus planning process. The process mapping effort for this applied study was adjusted from the entire campus design process to large-scale capital projects that have substantial impact to campus neighborhoods.

Professionals in project management create many kinds of process maps identifying the interaction of time, labor, work sequencing, work dependencies, and funding. It is a widely accepted business practice and advocated as a means to analyze various business functions. Facilities professionals often use similar industry-standard processes, as do construction project managers, in order to produce buildings on time, in scope and in budget - the three indicators of success for project managers. Process maps work well for direct and linear decisions - after all a roof cannot be put on a building before the foundation and support walls are up. These decisions and timelines often fall into "swim lanes" with clearly delineated boundaries, labor, materials, and dependencies.

Utilizing process mapping for complex systems thinking is a means to separate the areas of responsibility and decision making. In Figure 2 the process for the first year of a University of Wisconsin-Madison capital project is shown in swim lanes. The top lane is the University, the middle lane is the University of Wisconsin-System/Board of Regents, and the bottom lane is the State of Wisconsin (which includes the authorizations required by the state's administrative agencies, the legislature, and the governor). Each level of bureaucracy is designated a lane and the processes are placed along the timeline. This provides a temporal graphic of the approval 
sequencing and areas of responsibility. This process map represents the command-control aspects of the process, the points at which public meetings and approvals are needed to move the project through the system. It also allows for an assessment of the efficacy of any change to the process, which enables iterations of process change through which best practices can be integrated with regularity.

Figure 2

Capital Project Process Swimlane Diagram

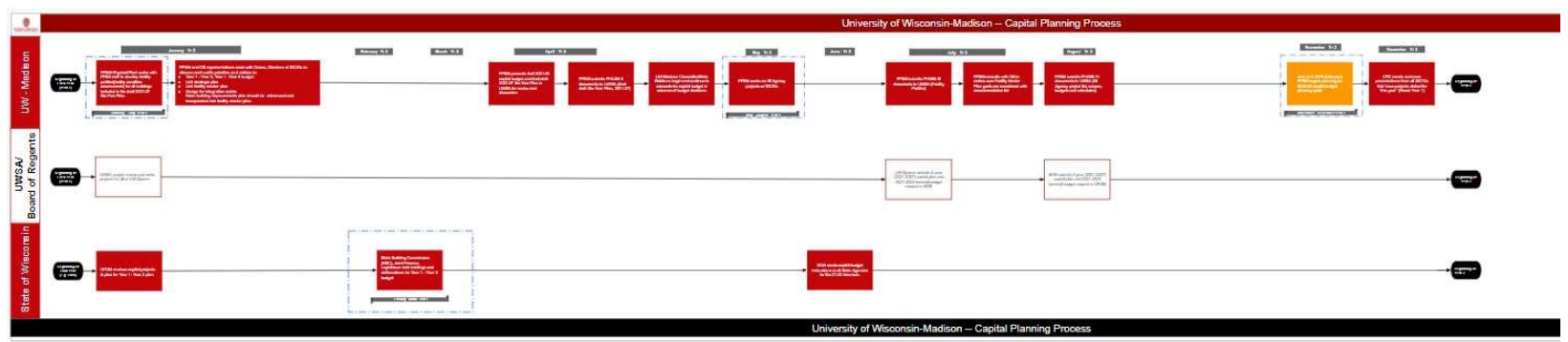

Note. A swim lane diagram which shows the project approval process along each of the vertical hierarchies. The top lane is the University, the middle lane is the University of WisconsinSystem/Board of Regents, and the bottom lane is the State of Wisconsin (which includes the authorizations required by the state's administrative agencies, the legislature, and the governor).

Another form of process mapping, along a longer time continuum is shown in Figure 3. This timeline is used frequently in capital project presentations at the University of WisconsinMadison to demonstrate the approval and authorization process and timelines for each biennial proposal. The State of Wisconsin budgets for capital projects in two-year increments (referred to as the biennial budget) and plans in six-year increments (referred to by the years the budget encompasses). The swim lane process map shown in Figure 2 breaks down the process shown in 
Figure 3 to a more granular level. This enables the level of analysis needed to understand where and when it would be feasible to transform the project process to accommodate the attributes for learning, wellbeing, and equity.

Figure 3

Capital Budget Process Timeline

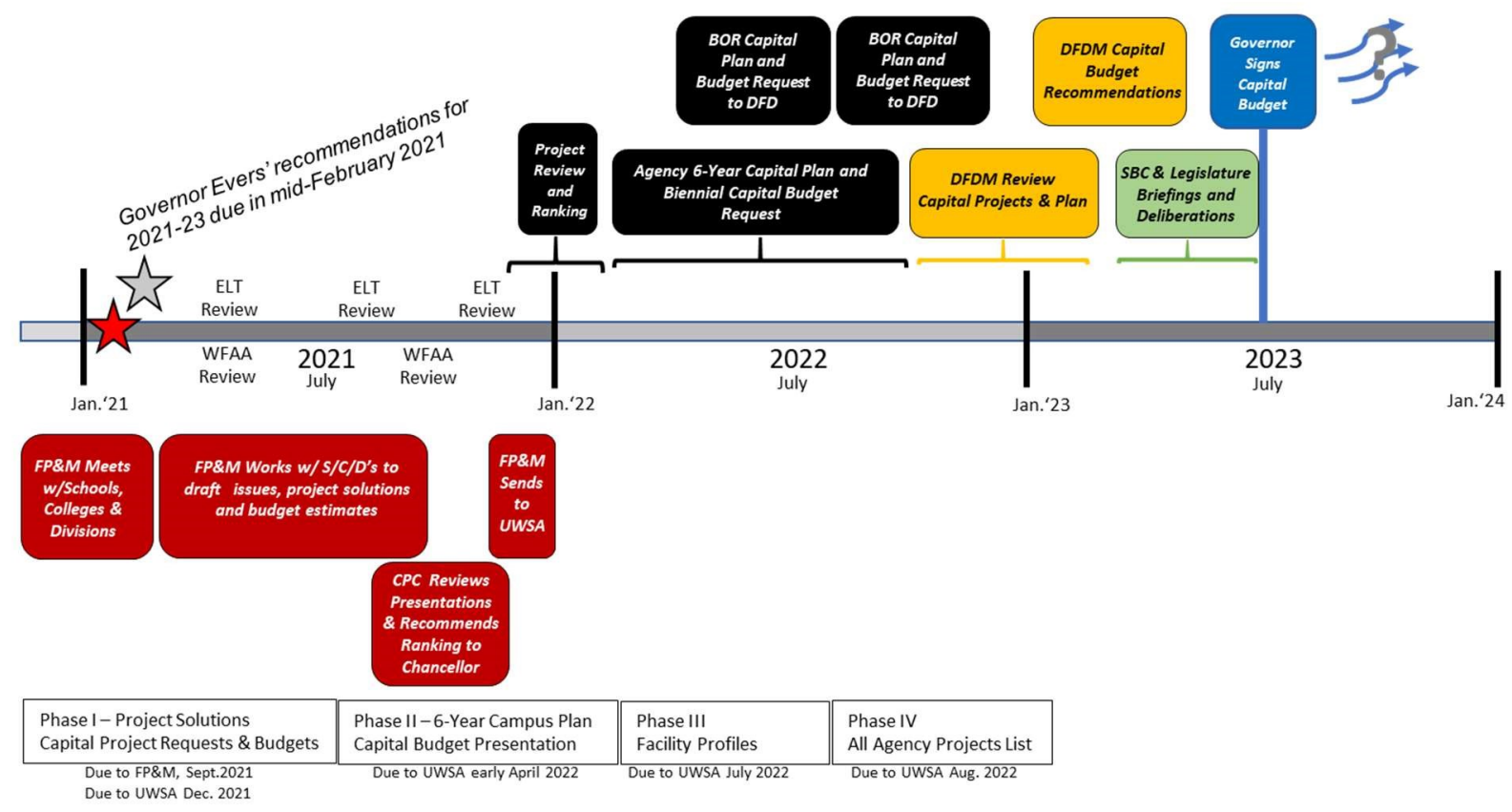

Note. The capital project budget timeline for the 2021-2023 biennium denoting the points in time that each agency in the vertical hierarchy has decision points.

\section{Incorporating Design Principles into the Capital Planning Process}

The processes involved in campus design and construction are continuously running on a loop. At the University of Wisconsin-Madison there are hundreds of projects at various stages of 
the process every year, and as each project progresses through to completion, other projects enter the queue, creating a non-stop cycle of activity. Much like sticking your hand into a spinning wheel, this makes it very hard to interject a new process so the approach I took was to interject new principles for design. I did not replace the existing principles such as designing for energy efficiency or stormwater management, but instead formalized the values set forth by the University's strategic framework and added them as design principles.

The premise of design principles is to "thoughtfully consider" them at the beginning of every project and include them in the project wherever it is feasible and within scope (AIA's Framework for Excellence, n.d.). In this study, these are the high-level constructs of Design for Learning, Design for Wellbeing and Design for Equitable Communities derived from the AIA Framework for Excellence and the UW-Madison Sustainability Guidelines. As principles of design these constructs can be manifested in different ways depending on the project. Designing for equity may include gender neutral restrooms, a certain pattern of carpet tile, or potty areas for service dogs. There is flexibility in every project to incorporate the design principles differently. The point is to think hard about how these principles will be experienced and actualized in the built environment, and to incorporate them into the design. This was an important point of consideration in determining the entry point for the integration process and resulted in modifications to the process for the second set of capital projects (State of Wisconsin, Division of Facilities Development and Management, 2020), as well as the addition of another study to examine the integrated design of classroom spaces.

High level constructs such as design principles was not something that the facilities project managers were accustomed to, they wanted to be told what to include in the project, 
preferably in the form of a checklist. Their jobs are not to design the project, their jobs are to ensure that the project is delivered on time, within scope, and within budget. Checklists, consistency, and boundaries facilitate their work, whereas innovation and change confound it. Incorporating design principles that promote variability makes their objectives more difficult to manage and achieve. To ease the burden for these initial pilots, it was necessary to limit change in order to manage the processes effectively. The literature identified several attributes of the environment that were most impactful on learning, wellbeing, and equity. For purposes of this applied study, I selected the attributes of lighting, nature, color, and informal spaces. I also prioritized "integration" - getting the voices of more stakeholders into the design process. Throughout this study I will refer to all of these as attributes. These attributes were referenced against the emerging building designation systems (e.g., LEED, WELL, etc.) to identify which were most commonly being incorporated and measured. Many of the attributes positively impacted all three of the imperatives of the design principles (learning, wellbeing, and equity) and were then prioritized for this change management project and provided to the project managers as checklists. Subsequently, the point at which each attribute was included in the capital project was then identified in the process maps.

Another modification to the method had to do with identifying what process would be most suitable for an integration study. It was also discovered that the granularity of some of the attributes did not fit in at the high-level capital processes, they fit better within the project design process. Yet, it was imperative for some of the attributes to be considered at different points along the temporal capital planning continuum before the project design phase began. Even some very technical attributes, like the Kelvin standard for exterior lighting, had to be addressed 
with a different institutional process before going out for bid. To change the lighting standard requires a change in the Campus Standards. The Campus Standards are a set of documents used in the capital planning process to inform the Architectural/Engineering firm of what the campus expectations are for mechanicals, furnishing, fixtures, etc. for each and every facility. This allows the A/E firms to develop accurate cost estimates which they use to bid on the projects. However, the lighting standards are developed by the campus electricians, university police, and another agency of the State of Wisconsin, none of which are involved in the capital planning process. These types of discoveries required an adaptive and flexible approach in the project.

\section{Learning From Others}

The peer institutions for the University of Wisconsin-Madison for this study are the fourteen universities which comprise the Big 10. There were two rating/ranking systems that were referenced to ascertain which of the Big 10 institutions were performing better than the University of Wisconsin-Madison; the HERD Report (Higher Education Research and Development Survey) and STARS (Sustainability Tracking, Assessment and Rating System). The HERD is a key performance indicator for R1 institutions as a metric of research expenditures. It is also a focal point for the University of Wisconsin-Madison as the institution's ranking has fallen from $3^{\text {rd }}$ to $8^{\text {th }}$ since 2010 (National Center for Science and Engineering Statistics, 2021). STARS is a national reporting tool comprised of 63 relative indicators across four categories: Academics \& Research, Engagement, Operations, and Planning \& Administration (Association for the Advancement of Sustainability in Higher Education, n.d.). The STARS report is also a focal point for the University of Wisconsin-Madison and is a reporting tool that captures data in several categories that are relevant to this study. 
The HERD ratings are broken down into sources of research funding. As noted, when the universities are ranked by all fund contributions, the UW-Madison ranks $8^{\text {th }}$. However, when ranked by business funding (sponsored research), the ranking falls to $54^{\text {th }}$ (National Center for Science and Engineering Statistics, 2021). This is obviously an area of opportunity, which has not gone unnoticed by university administrators. The HERD provides data sets that allows for robust analyses of research funding. Similarly, the metrics that comprise the STARS ratings demonstrate areas of opportunity. To analyze the STARS data required a different approach since the tool has multiple iterations and the criteria can change from one iteration to the next. The ratings are good for three years so there is variability in the age of the data, and there is some subjectivity to some of the criteria.

To review the STARS data, the institutional averages for the Big 10 schools were calculated and put in a heat map to allow for a quick visual distillation of where the UWMadison sits in relation to peers. This was done for each of the credit areas. An analysis of the Academics \& Research credits is shown in Figure 4. The heat map uses red to demonstrate where the UW-Madison scored behind its peers, yellow indicates neutral, and green indicates where the institution scored better than its peers. Of particular interest to this study were several select outcomes for academics, operations, and engagement. 
Figure 4

Example Display of UW-Madison STARS Academic Rating Comparisons

\begin{tabular}{|c|c|c|c|c|c|c|c|}
\hline & & & & $\begin{array}{l}\text { Total Points } \\
\text { Available }\end{array}$ & UW-Madison & $\begin{array}{l}\text { Peer Group } \\
\text { (Avg) }\end{array}$ & Difference \\
\hline \multirow{11}{*}{ Academics } & \multirow{8}{*}{ Curriculum } & $\mathrm{AC} 1$ & Academic Courses & 14 & 5.88 & 9.79 & 3.91 \\
\hline & & $\mathrm{AC} 2$ & Learning Outcomes & 8 & 1.06 & 3.08 & 2.02 \\
\hline & & $\mathrm{AC} 3$ & Undergraduate Program & 3 & 3.00 & 3.00 & 0.00 \\
\hline & & $\mathrm{AC} 4$ & Graduate Program & 3 & 3.00 & 3.00 & 0.00 \\
\hline & & AC 5 & Immersive Experience & 2 & 2.00 & 2.00 & 0.00 \\
\hline & & AC 6 & Sustainability Literacy Assessment & 4 & 0.00 & 1.71 & 1.71 \\
\hline & & $\mathrm{AC} 7$ & Incentives for Developing Courses & 2 & 0.00 & 1.81 & 1.81 \\
\hline & & AC 8 & Campus as a Living Laboratory & 4 & 4.00 & 3.94 & -0.06 \\
\hline & \multirow{3}{*}{ Research } & $\mathrm{AC} 9$ & Research and Scholarship & 12 & 5.65 & 10.28 & 4.63 \\
\hline & & AC 10 & Support for Research & 4 & 3.00 & 3.76 & 0.76 \\
\hline & & $\mathrm{AC} 11$ & Open Access to Research & 2 & 0.00 & 1.14 & 1.14 \\
\hline
\end{tabular}

Note. Example of how the peer ratings for the Sustainability Tracking, Assessment, and Rating System's Academic credits were displayed as a heat map to highlight UW-Madison's deficiencies to stakeholders.

\section{Trial and Error: The Implementation and Analysis}

The final steps in the methodology for this project were focused on implementation and assessment as an iterative process - this was the applied portion of the project. These steps were undertaken in two phases. The first was to select an integration method that could be used as a pilot for the university. The integration framework that was selected is based on the American Institute of Architect's Committee on the Environment Top Ten model (American Institute of Architect's, n.d.). This particular framework was widely used and accepted in the construction industry and contained the attributes and metrics that most closely aligned with the identified project outcomes. It also incorporated assessment as a guiding principle, and this was critical to 
informing and iterating the design process. I adopted this methodology for two capital project pilots and added two design principles specific to the university's mission: design for learning, and design for research. Thus, the initial pilots were referred to as the COTE Top Ten + Two and were used on an addition to the Veterinary Medicine complex, and a replacement for the Gymnasium/Natatorium.

Following the initial pilots, the State of Wisconsin's Department of Administration's facilities agency (the top level in the vertical hierarchy) adopted a modified AIA framework and mandated it for all state agency capital projects. The benefits of utilizing this particularly flexible tool lent itself to aid the state with its incredibly complex building portfolio. The framework can be applied to the building of a pit toilet or boat dock in the state parks, a maximum-security medical ward at a prison, and a chemistry building or residence hall at a university campus. This flexibility also aided this dissertation project, given the varied building uses and academic cultures involved in the capital project process.

\section{Study Process}

The study process required mapping processes for facilities construction and space design. Along the mapped pathways it was necessary to identify possible intersections of decision or influence which could be attributed to roles/positions, cost, departmental policy, university policy, system policy, and municipal, state, or federal law. The identification of these decision and influence points was critical to the assessment. At points where the process advanced or halted I was able to identify if the obstacle or accelerator was a person, a departmental or institutional priority, a policy, or a law. There were two additional aspects of 
the study, internal processes (analyzed through a classroom redesign study), and unintended consequences of internal processes (analyzed through a lighting accommodation study).

It was necessary for the pilot study to identify the attributes of spaces, buildings and grounds which had been identified as having a positive correlation with learning, wellbeing, and equity. Existing metrics for these attributes were identified and served to provide assurance to the project teams that there was something they could note in their reports and status updates (related to the desire for check-off boxes). Through the mapping of the existing design process, a modified integrated design process (based on the American Institute of Architect's Design for Excellence framework) was implemented. The integrated process established formal processes for additional stakeholder input. For these projects, the stakeholders were primarily students and faculty, with some community/client input. The AIA framework incorporates ten design principles, and for the purposes of the higher education mission, principles of learning and research were added for a total of twelve design principles. These additions were critical to center the university's mission into the design of the projects.

A functional aspect of the study was the impact to operational efficiency and institutional cost. Although actual metrics from the building operations were not part of this study, there are known operational deficiencies attributed to the current design process that this study attempted to mitigate. One of these areas was lighting and accommodation services. Lighting is generally selected for energy efficiency to meet an operational cost objective, and measuring the effectiveness is reflected in both financial metrics and energy metrics. However, lighting is only selected for how it impacts the electrical meter, with standard colors and temperatures established through industry recommendations, creating an environment that 
is often in conflict with the best lighting for learning, wellbeing, and equity - critical impacts which are not reported in fiscal metrics, mechanical meters, or student end-of-semester evaluations. This study attempted to integrate lighting standards that are positively correlated with learning, wellbeing, and equity. A parallel process mapping study of classroom lighting processes helped to inform the design principles.

\section{Types and Sources of Data}

For this pilot study, the University of Wisconsin-Madison served as the host implementation site, and an R1 institution of similar size, function, and vertical organizational hierarchy (within a state university system) was identified for purposes of comparison. Data sets related to the physical attributes of campuses are plentiful, and for this study I used three specific reports.

1. Sustainability Tracking, Assessment and Rating System (STARS). A national assessment system comprised of 63 rating criteria across four campus areas: planning and administration, engagement, academics and research, and operations.

2. Facilities reports from Gordian. Gordian is a private company that has proprietary facilities data from clients and then uses the data to provide clients comparative information

3. Facilities Condition Index (FCI) reports. Reports that assess the maintenance, repair, and replacement deficiencies of the built environment. These reports are often used to site the backlog of deferred maintenance in financial terms.

It has been noted by the Institute for Higher Education Policy (IHEP) that university ranking systems have "inherent weaknesses" which "often overshadow their strengths," and 
that the methodologies produce a single number which dilutes key characteristics and aspects of institutions (IHEP, 2007). Since the focus of this study is an R1 institution within a state university system, the data sets that I used are related to the key performance indicators in the areas of research and state performance metrics. In universities with very high research additional measures are critical to securing external revenue streams to support campus operations through indirect costs (the indirect administrative and infrastructure support required to conduct research). The data sets I used for this study are:

1. National Science Foundation (NSF), Higher Education Research and Development (HERD) data.

2. State of Wisconsin, Outcome Based Funding 2020 Forward Strategic Framework report card.

3. Sustainability Tracking, Assessment and Rating System (STARS) reports.

During this study, the AIA COTE was renamed to the Design for Excellence framework and this tool also includes design criteria and access to datasets of national measures in each of its ten design principles.

It may seem that this is an over-abundance of data which makes this study unwieldy, however it is important to recognize that a systems approach requires integration of disciplines - and of data. A key aspect of this study was to identify which data sets are used, and how the data is used, to inform decisions at each step of the processes. For example, facilities personnel use specific sets of data to inform decisions, while Deans use different sets of data to identify programmatic needs. Incorporating new data sources and methods into the process is not actually part of the formal campus planning process, hence the need for this applied study. 
Working with the knowledge that facilities performance, institutional efficiency and financial metrics are different than the metrics and indicators for academics, wellbeing or equity, the study required mixed methods to gather data that is relevant to the programmatic and operational decision makers of campus space design. Metrics and indicators that are gathered for institutional reporting purposes through the State of Wisconsin's outcome-based funding, accrediting bodies such as the Higher Learning Commission, and other relevant performance data related to energy and maintenance, accommodation services, sustainability, student life, and health services are also referenced in this study.

\section{Research Method}

For the purposes of the study, I used select attributes of the environment which have been correlated to impact learning, wellbeing, and equity, and for which I was able to obtain data. There are several research methods available to transdisciplinary researchers, and in this particular study I used the approach developed by Thomas Jahn to address a real-world focused approach (Bergmann, et al., 2012; Jahn, 2008). This approach is used to address societal problems that serve a public interest, and the goal is to produce an outcome that can be applied as a solution (Bergmann, et al., 2012, p 32). In this approach the research objective is developed through an integrated team of stakeholders which includes subject matter experts as well as the users and programmers of space, also referred to as societal actors (Bergmann, et al., 2012, p 14). The results included strategies, concepts, measures, and prototypes which were implemented at an R1 institution.

This approach utilized the functional research methods of the engineering and medical sciences as well as the hermeneutic paradigm of the social sciences to capture the physical as 
well as the socially constructed aspects of learning, wellbeing, and equity (Jackson, 1982). Suskie's (2018) rating system for evaluating college-wide student learning is replicable to this approach and helped to frame how scalability can be addressed. For example, using existing correlational data on how various physical space attributes impact equity, we can assess how a combination of those attributes can directly impact equity in a given space. For classroom lighting, surveys of the students were conducted for all classes in the spaces, and data was provided by the various accommodation services staff.

\section{Summary}

Having data for space attributes and their correlation to learning, wellbeing and equity enables procedural changes both in the process of space design, and in the campus standards and guidelines for new and remodeled spaces. Current UW-Madison design standards address codes, aesthetics, environmental stewardship, universal design, energy efficiency, durability, and performance of materials (UW-Madison Campus Design Standards, 2017). The impact on learning, wellbeing, and equity (other than ADA requirements), is not a factor in capital projects, campus remodels or deferred maintenance activities. While these principles are referenced as values to the institution there is no method for integration, setting standards, establishing metrics, or assessing effectiveness.

An intentional and focused assessment of spaces aligns the operation of campus with its mission and demonstrates effective stewardship and accountability of resources. Scaling that methodology to all areas of campus, including the natural areas, allows for a holistic and evidence-based approach to short and long-range institutional planning to achieve multiple objectives that are core to the mission of the institution. Ideally, the integrated processes and 
revised campus standards situate the institution for consistent application of a systems approach.

This integrated process provides regular collaboration among many disparate areas of the campus to optimize administrative and operational efficiency. Additionally, an environment designed for learning, wellbeing, and equity may accelerate a culture in which these embedded values are a core consideration in the design and construction of the campus. It will simultaneously assert a reputational brand that the institution is committed, innovative, and fiscally responsible.

This assessment model presents an opportunity to bridge the divide between the pure physical functioning of the campus, and the actual learning needs of diverse populations and communities. With the data from this applied study, it is possible to develop data-driven recommendations on how our building spaces could be designed for optimum performance and contribution to the institution's core values. Furthermore, the initial pilot processes are able to be modified and enhanced to capture data on other spaces and attributes - interior and exterior - in order to truly optimize the campus environment in a meaningful and deliberate manner. 


\section{CHAPTER IV: MAKING SENSE OF SYSTEMS}

\section{Introduction}

Guided by four research questions, I set out to examine how the design of higher education campuses could intentionally incorporate attributes of the built environment that contribute to learning, wellbeing, and equity. The research protocol was established to identify organizational structures that impede or facilitate the process, available metrics, and a means to define success. To begin, I examined the organizational structure of two R1 universities, and mapped the campus design process through those structures. I then identified industry accepted attributes and measures for learning, wellbeing, and equity. Next, I examined the documents, meeting artifacts and correspondence related to several capital projects to determine if, when and how the imperatives of learning, wellbeing and equity were manifested in the project design

process. Finally, I implemented an integrated design approach on two capital projects and one classroom remodel study. Following an assessment of the pilots I made modifications and implemented a second iteration on two more capital projects. Concurrent to the capital planning integration processes I conducted a detailed process mapping on the accommodation process for lighting impacts as a focused study on equity, an imperative in the campus design process.

\section{Findings and Results}

\section{Organizational Structures: State to Public University}

Selection of a comparable R1 institution to the University of Wisconsin-Madison was challenging, in large part due to the placement of the institution in the State of Wisconsin's bureaucratic hierarchy. While the University of Michigan is a peer in some areas, it leads the UW-Madison in many other rankings, and I selected it for comparison as an aspirational peer 
based on HERD rankings and STARS ratings. I began this research endeavor with a comparison study of these two competing institutions and discovered that the respective state budgeting systems and considerable autonomy, particularly the bonding authority legislated to the University of Michigan was a significant enabling mechanism that is not available to the University of Wisconsin-Madison. In fact, the University of Wisconsin-Madison is the only public R1 institution that does not have bonding authority (Meyerhofer, 2021). This made a comparison of the capital project processes with any otherwise comparable institution nearly impossible. Therefore, after analyzing the organizational structures, I opted to limit this applied study to the University of Wisconsin-Madison.

Two key differences between the University of Michigan and the University of Wisconsin-Madison are the additional layers of organizational control that exist in the State of Wisconsin, and the University of Wisconsin-Madison's inability to borrow. As noted in Figure 5, the State of Wisconsin has several layers of state government bureaucracy in the governance and funding of its public institutions. Whereas the State of Michigan has placed the governance of its R1 institutions (University of Michigan, Wayne State University and Michigan State University), in the hands of voters (State of Michigan, 1963). 
Figure 5

Vertical Hierarchies of the Capital Project and Budget Process

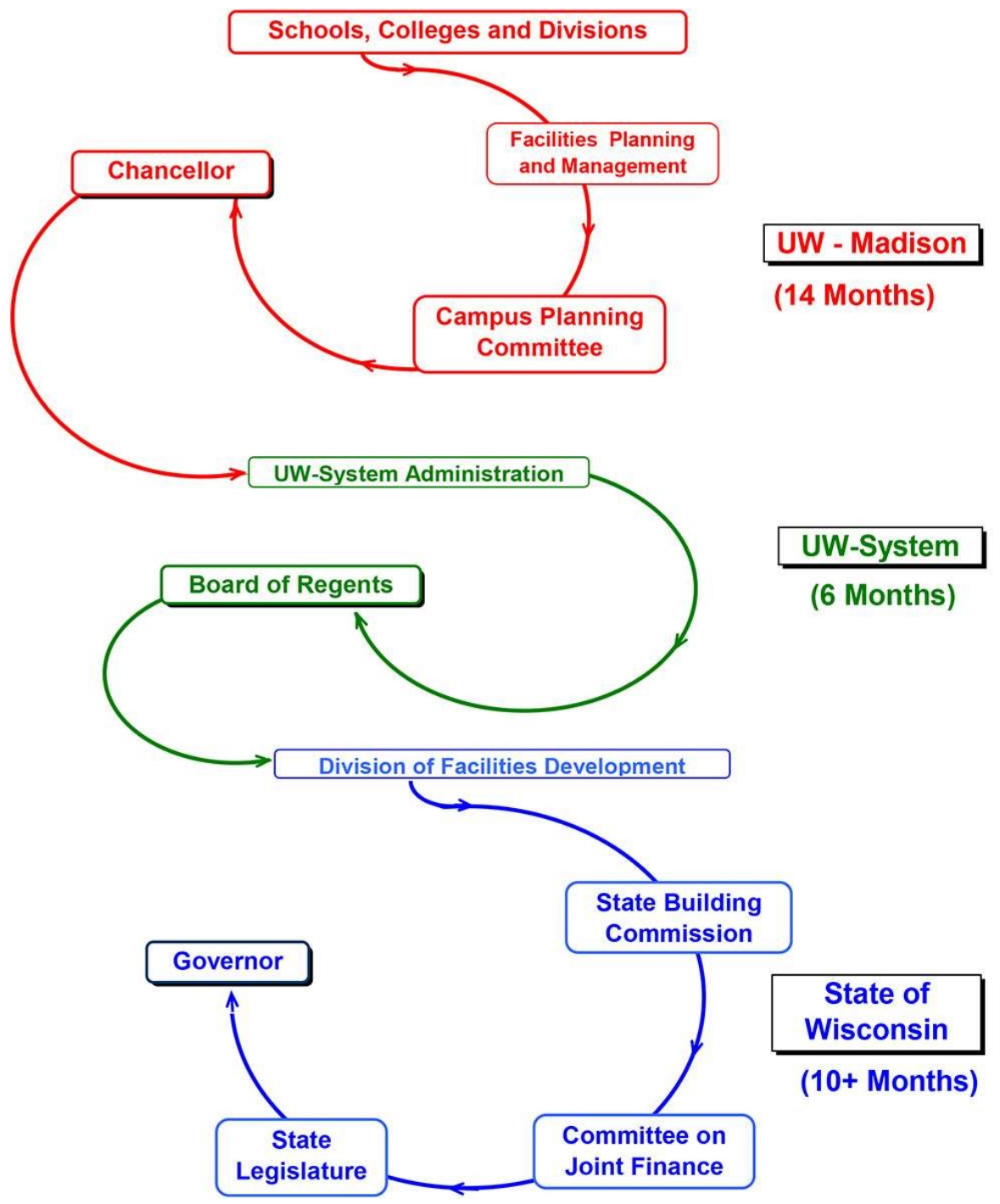

Note. The approval process for capital projects for the University of Wisconsin-Madison. From Capital Planning \& Development, Facilities Planning \& Management, University of WisconsinMadison. (https://cpd.fpm.wisc.edu/capital-budget/)

Regarding funding capital projects, as seen in Figure 5 the University of Wisconsin- 
System schools, including its flagship university, are highly dependent upon a series of decisions within a vertical hierarchy for biennial funding allocations from the state, resulting in hundreds of projects submitted for state funding each biennium, with only the top few receiving funding. However, the University of Michigan notes in its Capital Project Guidelines that, "Due to the unpredictable nature of the State of Michigan Capital Outlay program and requirement for universities to match State capital outlay funds, the University depends on other primary sources of funding to fund its capital projects" (p. 14). Hence, there is generally only one project presented to the State of Michigan's Capital Outlay program annually.

For comparison, in the FY 21-23 Capital Budget Process, the University of WisconsinMadison submitted 128 projects for capital funding totaling $\$ 345,985,700$ (University of Wisconsin-Madison, 2020). Whereas the University of Michigan submitted for a Computer Science and Engineering and School of Information addition (total authorized cost $\$ 145,000,000$; state building authority share $\$ 29,999,800$; university share $\$ 115,000,000$; state general fund/general purpose \$200) (State of Michigan $100^{\text {th }}$ Legislature, Senate Bill No. 748). In addition to the differences noted between these two flagship institutions, the University of Wisconsin-Madison's capital projects list also must compete with the capital projects requests from the other twenty-five post-secondary institutions in the UW-System. The UW-System level of the vertical hierarchy prioritizes the combined capital requests and forwards those to the State for budget approval. Of the 128 projects advanced by UW-Madison, only four construction projects and two advanced planning requests were submitted for budget authority to the State of Wisconsin (University of Wisconsin-System, n.d.). 
Clearly, the differences in state governance structures and legislated authority negate any legitimate capital project comparisons of the organizational hierarchy levels between the respective universities and their state governments. However, what is also important to recognize is that the imposition of additional hierarchical controls in Wisconsin results in the need for increased informal networks at the University of Wisconsin-Madison. Alvarez and Ferreira (1995) identified the inverse relationship between deep bureaucracies and the need for informal networks as organizational systems move from hierarchical to flat. In functional terms, among horizontal organizations there is a proliferation of informal networks that are more adaptive, flexible, and spontaneously developed. Horizontal organizations expend their time and resources building informal networks that are responsive to their mission, instead of expending those same resources influencing decision makers along the vertical hierarchical structure. Organizational Structures: Impeding \& Enabling

The University of Michigan and the University of Wisconsin-Madison have significantly different state-to-university structures. Given the deep vertical bureaucracy in Wisconsin, it is expected that its flagship institution is likely to expend significant resources (both human and financial) toward the capital project process. Whereas the University of Michigan is benefited by being able to leverage their resources and networks toward their mission. As noted in the literature review, and evident throughout this research study, higher education organizations are highly decentralized. Universities are comprised of many different, largely autonomous, entities acting as independent agents, and this makes it difficult to advance a central cohesive vision. Top-down edicts and planning efforts only go so far in this type of environment. In describing how the University of Wisconsin-Madison advances core institutional values, the Chancellor's 
Chief of Staff noted in a meeting of the Sustainability Advisory Council (2021) that, "There is an art of both setting the vision and then harnessing the power of a decentralized management structure" (M. Maryl, personal communication, April 9, 2021). The Chief of Staff went on to note that the opportunity to gather information from lots of bright people is empowered through informal networks. "The way she [the Chancellor] drives change informally is in the consistency of the questions she asks" (M. Maryl, personal communication, April 9, 2021). Having leadership continuously raise the same questions in conversation with different networks has a multiplier effect. Across a variety of internal and external organizational relationships, the connections engendered among adaptive organizational systems are demonstrated to be different and more dynamic than structured hierarchical connections (Aalbers, Dolfsma, \& Koppius, 2004; Gulati, Dialdin, \& Wang, 2002; Song, Temby, Kim, Cisneros, \& Hickey, 2019).

To understand the adaptive organizational system, it is necessary to know the formal structures and processes. This research study specifically analyzed the formal processes of the capital budget process to provide clarity to the structure with the intent of identifying the roles, processes and policies related to designing for learning, wellbeing, and equity. The research demonstrates that while there are design attributes and measures for learning and wellbeing, the attributes and measures for equity are an emerging field of study. However, there is some consensus that attributes can contribute to positive outcomes for all three imperative constructs (Clauson, 2018; Holmes, 2018). The constructs of learning, wellbeing and equity are very interwoven, and it should be expected that a single attribute, such as art, can influence all three. Similarly, the informal networks of art aficionados, can influence processes across the organizational hierarchies. Curated collections of art, or philanthropic donations of art included 
in the campus design process, has a multiplier effect of serving both the design principles of a learning environment, and the process of project prioritization.

This type of influence was evidenced in the Veterinary-Medicine addition project. The School of Veterinary-Medicine has an extensive curated art collection, all related to animals. The philanthropic arm of the School is responsible for the curated collection, as well as responsible for raising a significant portion of the funds for the project. It also curates relationships with donors. High-capacity donors generally also have relationships with legislators, or partners of legislators, and move together in social circles where these informal influences occur. The art collection at the School of Veterinary Medicine serves to provide a learning environment that contributes to a culture of wellbeing and equity, and it serves as a pathway to influence hierarchical processes through the adaptive organizational systems of informal networks.

The two universities, the University of Wisconsin-Madison and the University of Michigan, had different process flows, both internal to the institutions and external - particularly in the areas where the processes intersected with the state budgeting process. The governance and budgeting processes for these public, flagship institutions are relevant to the process analysis as an important note in understanding the expansiveness of informal networks in influencing capital budget processes. The University of Wisconsin-Madison is situated in a state with a university system, which has a governor appointed governing board, the Board of Regents. This creates a vertical organizational hierarchy as shown in Figure 5. 
The University of Michigan is independent from the state and has autonomy through the State of Michigan's constitution. The governance of the University of Michigan is through independently elected board members who supervise the school and its direction of funds (Constitution of Michigan of 1963, Article VIII § 5). The budget model at the University of Michigan looks significantly different, as does the organizational structure (Figure 6). While there are similarities between the University of Michigan and the University of WisconsinMadison's overall internal capital planning processes related to standard project development, the interactions with state governance are so different as to make them incomparable in the examination of the command-control structures. I will outline the key findings with the portion of the process related to state interactions. 
Figure 6

University of Michigan Capital Project Process

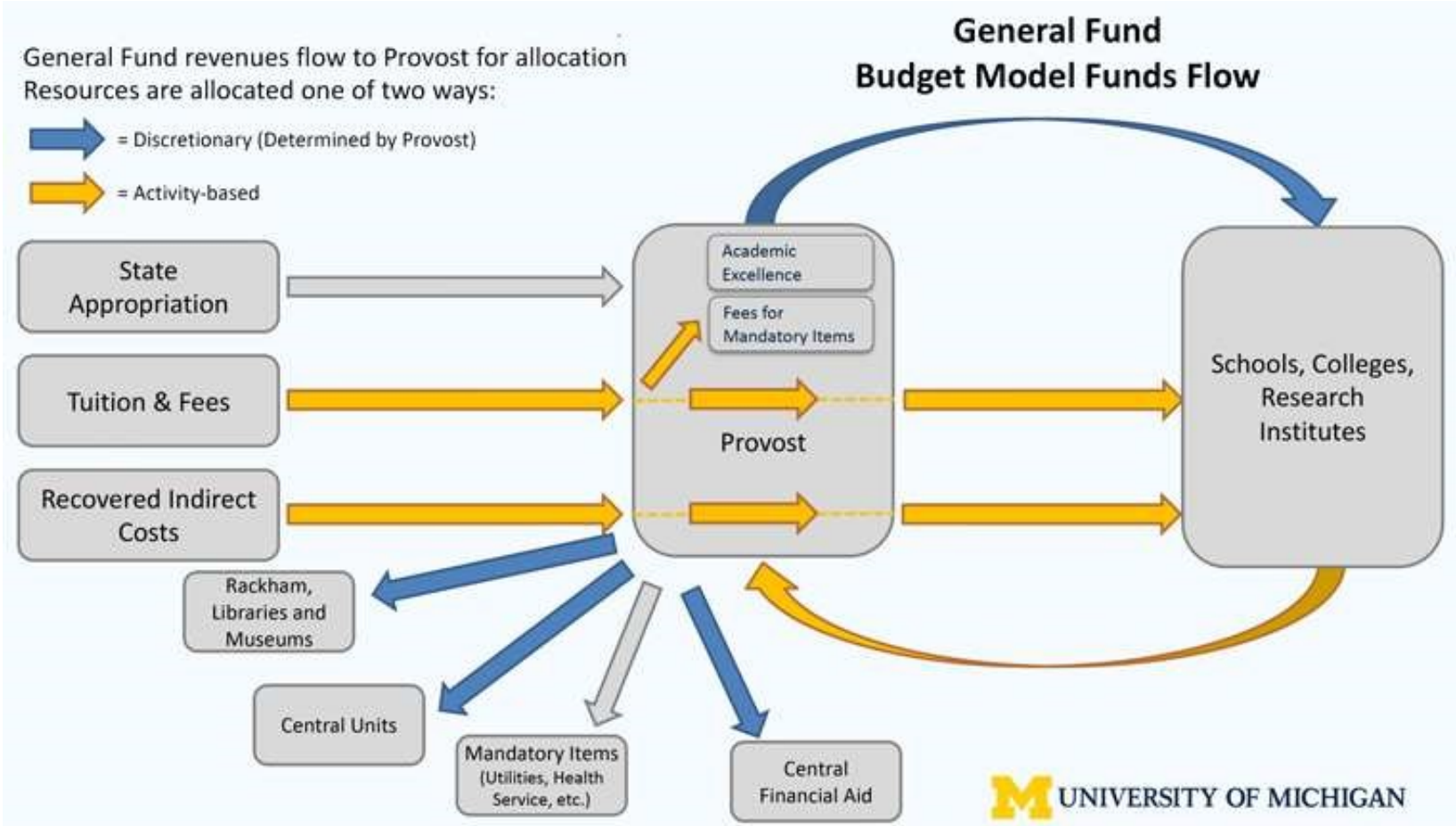

The additional authorizations of the vertical hierarchy in Wisconsin, and the timing of the State of Wisconsin's legislative processes can add significant time and costs to capital projects at the University of Wisconsin-Madison. As demonstrated in Figure 7, a donor funded project (which is exempt from the state's budgeting approval process), can be accomplished nearly four years faster. At an escalation rate of 3\% annually, a project enumerated for $\$ 150$ million could cost the state an additional $\$ 18$ million for a four-year delay. 
Figure 7

Example of Four Approval-Based Planning Scenarios for a Capital Project
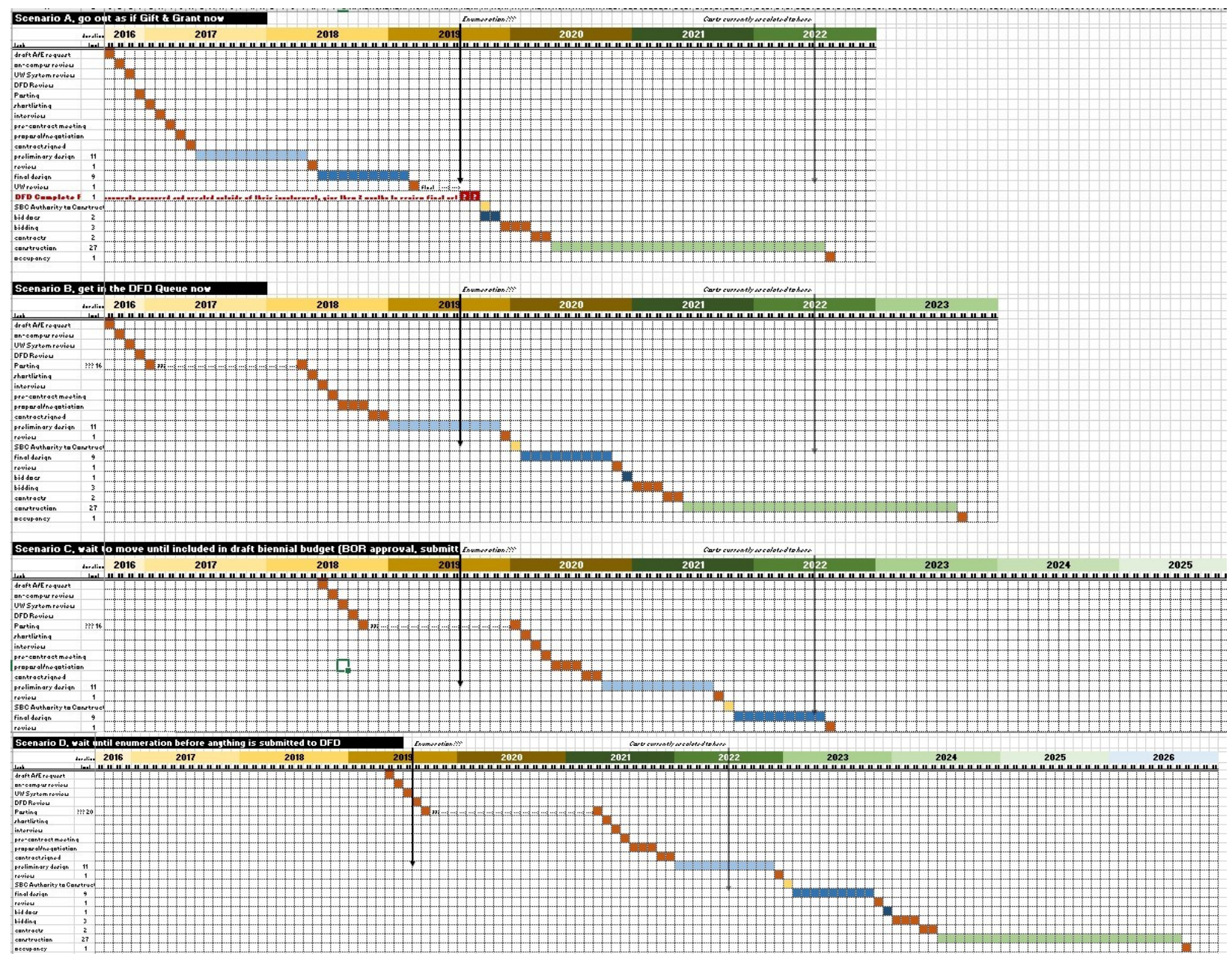

Note. The four projected project timelines above represent the temporal differences of project

completion based on funding authorizations. The timeframes are represented as a decade

beginning in 2016. The top timeline represents a 100\% gift/grant funded project with forecasted occupancy date of July 2022. The second timeline represents advancing the project immediately to get into the State's queue without formal enumeration. The third timeline represents waiting to 
be included in the State's Biennial Budget. The fourth timeline waits formal enumeration before advancing, resulting in an estimated occupancy of July 2026.

After reviewing these processes, and understanding the financial implications for any delays, there were two immediate hurdles that were identified. The first is that the money is always going to be tight and the longer a project takes the more that will have to be cut from the project (value-engineered). The second, is that introducing new concepts must be done well before a request is made to hire an Architectural/Engineering firm, and the concepts need to be reinforced, or even incentivized, as a priority throughout the project or specific attributes will be at risk of value-engineering.

\section{Applied Studies: Leveraging Organizational Systems for Campus Design}

The organizational relationships in this study were critical to integrating new concepts and attributes into a very incestuous process. I used the adaptive organizational system framework for this process, it fundamentally leverages personal and professional relationships to advance objectives through the hierarchical organization. Figure 8 provides a graphic that helps to visualize the interaction between the human relationships in organizations, and the organizational structures. In this model the connections that people have through professional or personal relationships create networks that embody the values, creativity and strategies that advance the mission of the institution. The ideas then must be approved, at which point they move into the traditional (or command-control) hierarchy. While this model shows these operating systems as separate, it is important to note that each model is comprised of the same people, just interacting in different ways. 
Figure 8

Relationship Between Adaptive and Traditional Organizational Systems

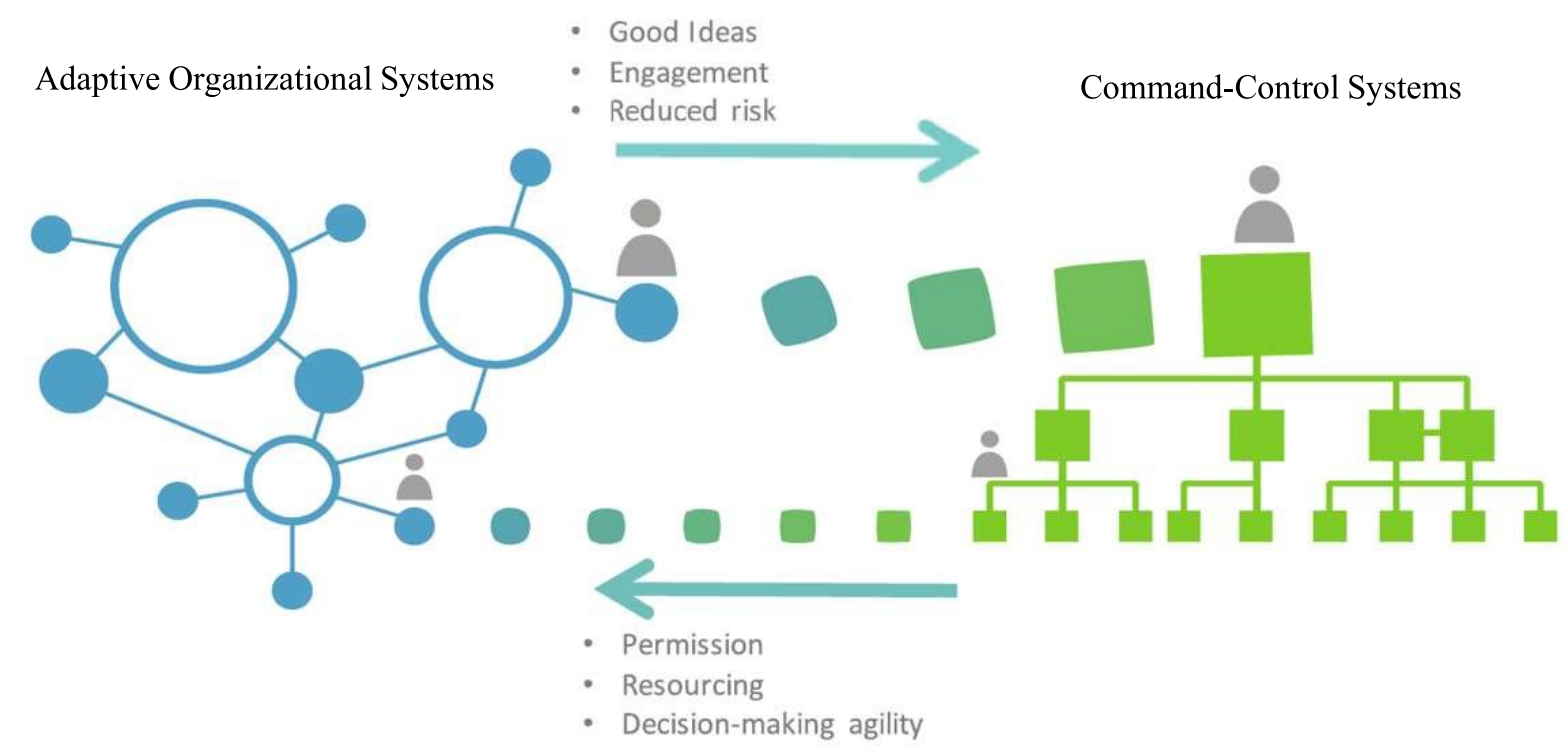

Note: “Optimize Synergy Between Both Operating Systems” by Leith Sharp is licensed for open sharing and adapting under Creative Commons.

This networking was crucial in the study. Given the movement of people into and out of positions throughout the vertical and horizontal hierarchies, it was almost inevitable that the power of interpersonal relationships would be the essential force needed to enact organizational change. In this study, the informal relationships that exist in the adaptive organizational system were the connections that bridged the vertical hierarchies of the command-control systems. These professional relationships resulted from people having worked in multiple roles throughout the UW-System, State Department of Administration, and the University of Wisconsin-Madison. This personnel movement was advantageous in two ways. The first is that 
it allowed people to learn the cultures and processes within each level of the bureaucracy. The second is that it allowed people to build relationships in each level of the bureaucracy. These are two areas of competencies that were critical to this study and directly supported the organizational change process. This study involved many people and leveraged their relationships, but it was fundamentally one person who had moved among the organizational levels and had professional affiliations and relationships over the span of four decades that was the proverbial drop in the pond. The ripple effect of this one person's network amplified, bringing awareness and synergies as it spread out. One of the focus areas of this study was relationships among organizational structures, it is important to note that the adaptive organizational system is not intended to be random relationships that are just happened upon, it is an intentionally curated system. It is very much a "connect-the-dots" analogy. Randomly connecting dots may or may not get the desired outcomes, intentionally connecting the dots in a coordinated manner will produce results. This is evident in the forensic analysis of the process maps.

\section{Forensic Mapping, Identifying Integration Opportunities}

To understand how, when, and where to integrate the new conceptual framework of designing for learning, wellbeing, and equity, it was necessary to map the existing process in order to understand the points of access and the obstacles along the way. To accomplish this, I used an analytical process co-created by Leith Sharp through a networked learning model that she coined Idea Flow. The Idea Flow process is open sourced and licensed through Creative Commons, enabling its use in any organization, and encouraging that it be adapted and shared as an evolving platform of knowledge. The Idea Flow framework "enables currents of information, 
creativity, ideas, capital and material to flow throughout our organizations and wider stakeholder ecosystems with increased agility, purpose and context sensitivity" (Creative Commons, n.d.). Forensic mapping is a component of Idea Flow which allows for an analysis of the flow of an idea or process through the adaptive and traditional organizational systems.

The purpose of a forensic mapping analysis is to dissect a previous project or process and investigate where it advanced, stalled, or failed along a timeline. Then the same timeline is placed along a continuum identifying when the project or process was in the adaptive organizational system and when it was in the traditional command/control organizational system. These data points allow for a more detailed understanding of enabling and disabling steps in the process, which helps the analyst to identify if those enabling or disabling points are a person, a process, a policy, or something else. This information is needed to address the source of a problem, or to identify a success which can then be replicated or used to inform other projects and processes.

I intended to analyze the project processes for the addition to the School of Veterinary Medicine and the Gymnasium-Natatorium projects, since these were the two initial integration pilots. I was brought into these projects immediately after the A/E was selected, to integrate sustainability into the projects. My intent was to use the university's project records to complete a forensic analysis of what had happened before I was brought into the process. However, what I discovered is that project records are maintained at different levels by different project managers. The university's project manager for the Veterinary Medicine Addition had kept incomplete records. Meeting minutes, project schedules, signed authorizations, and the results of vetting criteria were not included in the project files. So, I moved on to conduct a forensic mapping 
analysis of the second project, the Gymnasium-Natatorium, and discovered a similarly illmaintained university file record of the project.

The general folder structure adopted in 2019 for the university's Capital Planning and Development Group is comprised of 15 different folders. The folders, and a brief descriptor, was provided to me in an informal training on how capital project management files are maintained. Each active project has the same 15 file folders. All project files are maintained on a shared drive and each project uses the same set of file folder titles and organization. They were listed for me as follows:

00_BOR-SBC (Board of Regents and State Building Commission)

01_Contracts (Specific to the awarded AE...contract, CO's, Payments, etc.)

02_Intake \& Planning (Where the PM's do the bulk of the up-front work on the Project Budget Worksheet, Capital Project Request, Project Charter, Funding letter, etc.)

03_AE_Selection (this is where all the proposals/letters of interest should land)

04_Pre-Design (Reserved for background information, including past studies, and early programming if available)

05_Preliminary Design (A/E is under contract and producing things up until and including $35 \%$ documents - the bulk of info is in this folder)

06_Final Design (everything beyond 35\% and stopping at stopping at construction)

07_Bidding (as it implies)

08_Construction Admin (as it implies)

09_Close Out (as it implies)

10_Warranty (as it implies) 
11_Budgets and Schedule (used exclusively by the PM)

12_FFE (Stuff in the building that when flipped upside down would fall out)

13_Zoning \& DRB (often the best place to find presentations given to the public)

\section{4_WEPA EIA-EIS}

\section{5_Correspondence}

The contracted Architectural/Engineering firm's project manager is tasked with keeping all meeting notes and information, and the project managers representing the State, UW-System and UW-Madison are each charged with maintaining that information for their respective agencies. The above file format is specific to UW-Madison project managers, and as noted, was only recently adopted. My discovery was that some project managers are more proficient than others, and there are no clear or clean delineations about when a project is at certain threshold in the process. One project manager advised me that 'No project runs 'clean' where $10 \%, 35 \%, 90 \%$ align and relate to concept vs. programming vs. design development" (A. Williams, personal correspondence, 2020). I was also advised that due to the uniqueness of the projects, there are no uniformly applied file management standards, and that the client, funding source, and schedule will all vary. However, the university does adhere to the State of Wisconsin's Division of Facilities Development documents which provide some consistency across projects in a few standard forms.

In the vertical hierarchy in which the UW-Madison is embedded, each level of the bureaucracy has a project manager assigned to a capital project, so I probed the next level, the University of Wisconsin-System for record maintenance. I quickly discovered that a different file 
structure is used, and that as the projects progress through the processes, even the project numbers change as the projects move up and down through the hierarchy (C. Torstveit, personal communication, 2020). The same is true at the next level, the State of Wisconsin's Division of Facilities Development. The inconsistency in expectations of roles, personnel performance, file structure, communication and labelling, and data collection is significant across the organizational systems, and is a key finding of this study.

The discovery in the initial analysis that project managers have neither the time, training, nor expertise to be able to manage any additional inputs into the projects, required a pivot for the integration study. Any integration of institutional level design principles such as learning, wellbeing, and equity, were not going to happen with the project managers. Not only is there no capacity given their existing workload, but it is also clearly a conceptual framework that is outside of their scope of responsibilities. Project managers follow established processes, and I was told by many project managers that they just needed a check-off box and a clear set of instructions about which attributes needed to be included in their projects. With that clarity of communication, and since I was unable to conduct a forensic analysis of each project at a more granular level, I returned to an analysis of the system interactions at a higher level to find a more appropriate integration point.

\section{Stakeholders and Design Criteria}

In this applied study, I was often an active participant in the processes. I was brought into the two Capital Projects (Veterinary Medicine Addition and Gymnasium-Natatorium Replacement) at the point when the A/E firms had already been selected and the design discussions were just beginning. A/E firms bid on projects based on the RFP (Request for 
Proposals). The RFP is a document put out for companies to bid on doing the work. In order for a company to bid, the programmatic needs of the facility have already been identified and some rough orders of magnitude have been estimated through previous studies. In short, I was pretty much already late to the game at this stage of the process because the constructs of learning, wellbeing, and equity were not introduced to the campus design process, facilities condition assessments, or advanced planning processes until October of 2020.

Programmatic needs and studies, conducted years prior to project enumeration, informed the RFPs and the rating criteria through which the bidding firms were evaluated. This is important because the firms that bid on the project are evaluated on their experience and expertise in the identified program areas. For example, the Gymnasium-Natatorium Replacement has an ice rink, so some of the rating criteria centered around that unique design requirement. The Veterinary Medicine Addition also had specialized programmatic requirements for clinical space, an emergency room, vivaria, and wet and dry laboratories. However, nowhere in the RFP's or ratings criteria was a firm rated on their experience with incorporating learning, wellbeing, or equity into facilities design because these concepts had not been included in any part of the previous processes. Based on inquiries I made at the very first kick-off meeting for the Veterinary Medicine Addition, I determined that in order to integrate these concepts, I had to be involved significantly earlier in the process. The $\mathrm{A} / \mathrm{E}$ firm had not bid on any of these constructs, the sub-contractors had not bid on them, and the state enumerated budget did not include them, so they were deemed add-ons to the project and threats to the scope, time, and budget. However, because the capital planning process is perpetual, and there are usually over one hundred projects at various stages in the process, it was possible to be 
concurrently involved in several projects at different potential integration points. This proved to be extremely helpful in testing and piloting integration methods at different stages of the process, and to select project teams that had expressed interest in advancing these design principles.

The core project team is typically comprised of the project manager from the $\mathrm{A} / \mathrm{E}$ firm, the project manager from campus, the project manager from UW-System, the project manager from the State's Division of Facilities Design, and the project liaison from the School, College or Division. The roles of the School, College or Division's designated project liaison will vary by project and desire of the Dean or Director. The designated project liaison could be an Assistant Dean of Facilities, an Assistant Dean of Research, or in the case of Veterinary Medicine, the Associate Dean for Advancement - the area of specialization and familiarity with facilities can vary widely. While the design process is sequenced and has points along the process for stakeholder input, it is important to note that it is fundamentally five positions that determine the project outcomes, and three of them are generally specialized project managers for building construction. The integration of mechanical and electrical systems is something that construction project managers are trained for; the integration of cultural considerations and learning environments is not. There are professionals in interior design and classroom technology that are consulted at standardized points in the process, but the premise of learning or equity then becomes an attribute of a particular space rather than a design priority for a building, or a campus. The School or College project liaison will look out for their programs, and ultimately serve as the translator to ensure that the spaces will serve the program, but they will prioritize their School's program needs over institutional design principles. 
Arguably, the most qualified role on the core team is the A/E project manager, which is why the vetting of the $\mathrm{A} / \mathrm{E}$ firms is so critical. Most $\mathrm{A} / \mathrm{E}$ firms that bid on large university capital projects have impressive portfolios of similar projects they have done on other campuses. This helps to both inform each institution's project, and to ensure that there is continuous learning in higher education campus design. It also speaks to the power of the adaptive organizational system. It is general practice for the primary stakeholders of the Schools, Colleges and Divisions to visit other campuses and tour facilities to get some ideas of what peers or aspirational schools have done. The connections among A/E firms, Deans, Athletic Directors, and researchers provides an active learning network that helps to flesh out opportunities and emerging trends that can prove to be differentiators for an R1 university competing for top researchers and research dollars.

In the Veterinary-Medicine Addition project, the Associate Dean for Advancement and Administration served as the Project Liaison, while the Associate Dean of Clinical Affairs \& Teaching Hospital Director, and the Associate Dean for Research \& Graduate Training, were brought into the discussion for aspects of the facility that related to their programs. The Associate Dean for Advancement and Administration was responsible for coordinating the work and collaboration of all the School of Veterinary Medicine programs and keeping the Dean up to speed. The Dean would also periodically be brought into the meetings, particularly to discuss aspects of the design that would require a change to the scope, budget, or timeline. The work that happened within the School of Veterinary Medicine to gather input into the project was outside of the scope for this dissertation, but that work was critical to inform the project and substantially changed some of the design components - particularly from a logistics standpoint. 
For example, the new addition is going to house additional laboratories which will require transporting animals from the existing building through hallways to the new addition. The clinicians and researchers suggested that the connecting tunnel, that was originally proposed to be open and glassed on all sides, be split into two distinct hallways to allow for the movement of animals, researchers, and students to be separate from the public. A clinician's simple comment about having to gurney a tiger through the connector prompted a lively discussion related to public versus clinical and research space, which caused the redesign of the key components of the building from entryways to the tunnel, to access to the coffee shop.

For the Gymnasium-Natatorium Replacement project, the core team also consisted of the project manager from the $\mathrm{A} / \mathrm{E}$ firm, the project manager from campus, the project manager from UW-System, the project manager from the Division of Facilities Design, and the project liaison from the Division of Recreation and Wellbeing. The Division's project liaison for Gym Nat was the Recreation Sports Director. This project also had two student representatives on the core team since this was a student fee funded project. Students are not typically part of the process, which is a particularly salient point to consider when the new design constructs that are being integrated into the existing process are predominantly focused on student learning, student wellbeing, and student equity. In addition to the two student representatives, there was a concurrent student stakeholder engagement effort run by Recreational Sports, which they called the "Think Big Member Panel” (Recreation and Wellbeing, 2020).

My applied research study did not delve into the processes that occur within the Schools, Colleges and Divisions during a capital project, but it is important to note that that work is absolutely critical to this process. It is an area of the process that requires additional study 
because that is where the researchers and faculty work through the details of their programs and try to translate that into the built environment. I would argue that this is a critical piece of the process that needs further examination, particularly in light of how the unique cultures of each of the disciplines view the spaces of the built environment. Even shelving in offices is a differentiator, as some disciplines prioritize books over journal articles as an artifact of scholarship. Concepts such as the appropriate size for faculty offices, privacy of restrooms, security of laboratories, and advising space for graduate students varies considerably among the disciplines, and the lack of uniformity is a point of conflict in campus design and operations.

\section{Integration Pilots 1 and 2: Preliminary Design}

In the preliminary design phase of both the Veterinary Medicine Addition and Gymnasium-Natatorium Replacement projects, I was able to pilot the AIA's COTE Top Ten model, plus the two design principles that I added, design for learning and design for research. This addition to the preliminary design phase was enabled due to the informal relationships that existed among the $\mathrm{A} / \mathrm{E}$ firm and the three tiers of the vertical hierarchy. At a later point in this dissertation, I will elaborate on how this aspect of the adaptive organizational system facilitated the pilots of these design constructs, but first I will explain the design criteria that were adopted to demonstrate the significant overlap among the twelve criteria. The overlaps add value to existing design criteria but also create confusion, and some reticence, among the design team who are primarily construction project managers and engineers accustomed to a very standardized industry process. In summary, the additional design components were able to be incorporated into the process, but due to the disruption of standard practices, the constructs were sometimes viewed as burdensome and amorphous. The constructs that had quantitative 
measures fared much better in the process than those that were viewed as subjective or qualitative. To help contextualize the process, I will review some of the characteristics of the twelve design measures that were incorporated into preliminary design.

The COTE Top Ten (as it was named at the time of its use in 2019) is comprised of ten design criteria, some of which have quantitative metrics (e.g., energy) and some which are qualitative (e.g., change and discovery), and some of which are both (e.g., water). With the university's two additional design principles (learning and research) there are a total of twelve design principles as displayed in a presentation slide that I use with the Core Teams (see Figure 9). Each of the A/E firms for the projects approached this addition to their design processes somewhat differently, resulting in different outcomes. 
Figure 9

University of Wisconsin-Madison's Design Principles

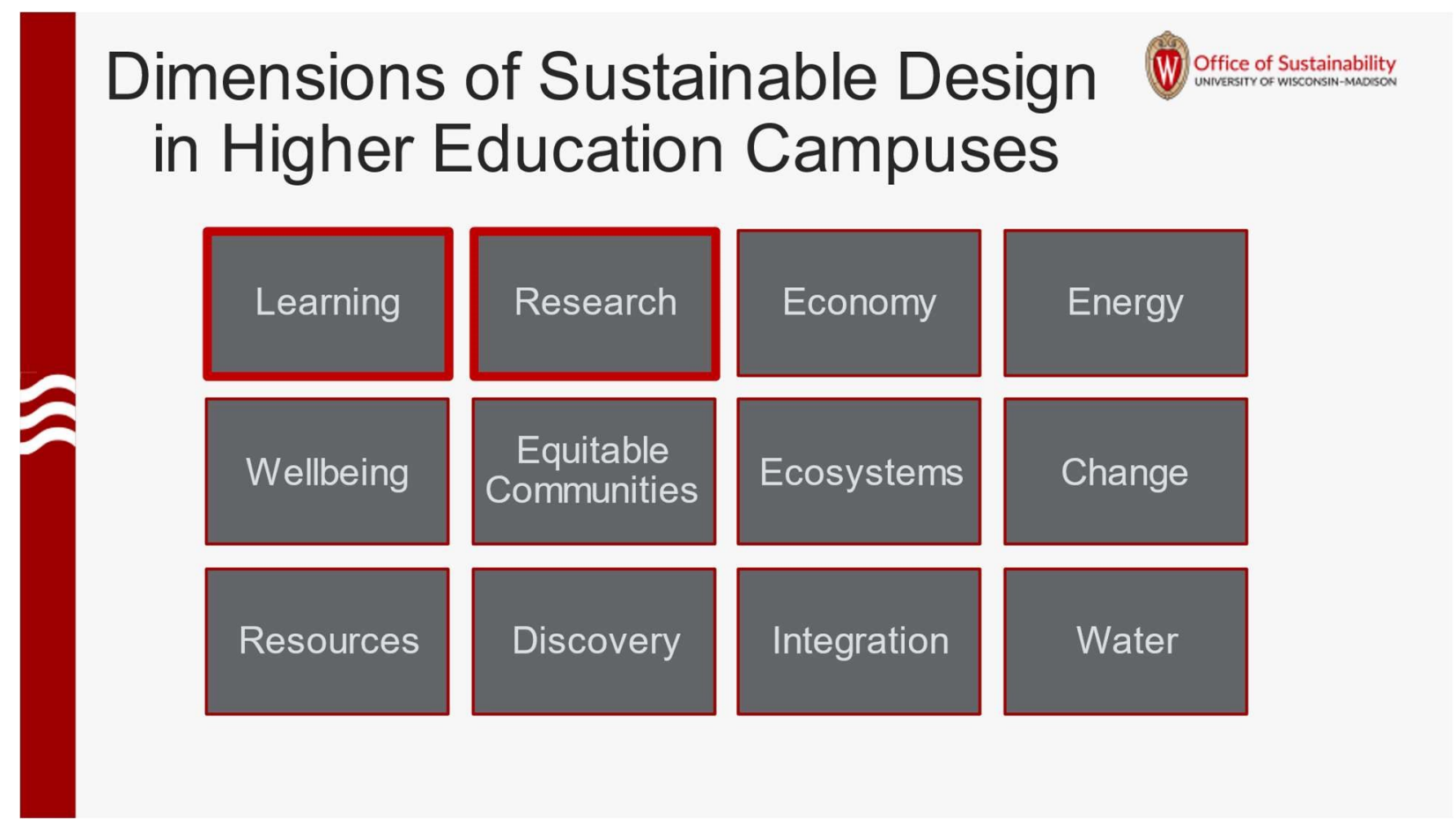

Note. The ten design principles established by the AIA, plus two additional design principles specific to the University which are highlighted in bold red outlines (University of WisconsinMadison Office of Sustainability, 2019).

The A/E firm for the Gymnasium-Natatorium Replacement project took the approach in preliminary design to conduct a sustainability charette. The Sustainable Design Criteria (as they were named for the pilot) were provided to the firm to inform their approach to meeting the university's priorities. In the meeting, the A/E's sustainability facilitator asked the core team to identify performance targets for energy, and construction and demolition waste diversion. These are typical for building metrics and the State of Wisconsin had established standards for these. 
During this one-time design charette, the A/E firm asked for additional guidance on the other design criteria, seeking to understand the performance metrics that they would be held to, and to understand the expectations of the campus. Throughout the discussion, the majority of the other design criteria were folded into existing design parameters, and there was no follow-up on these principles past the initial charette. Only wellbeing and equity persisted as distinguishable design concepts through the process and this was due to the student life and curricular programming, as well as the recent renaming of the Division from Recreational Sports, to Recreation and Wellbeing.

The Recreation and Wellbeing representatives were continuous advocates for wellbeing as a design priority. Equity was also advocated for, but the conversations around equity largely supported gender neutral locker rooms, and two clinical programs. For the clinical programs, the equity focus was limited to building access and movement through the building. There was a lot of discussion around the design considerations for one of the public-facing clinical programs in the School of Kinesiology which required accommodating the safety, mobility and transportation needs of clients with developmental and physical impairments. These equity discussions included the Kinesiology program leaders and the Associate Dean of Facilities for the School of Education, the home of the Department of Kinesiology.

The A/E firm for the Veterinary Medicine Addition took a different approach, one which was significantly more collaborative, time intensive, and inclusive. They also incorporated feedback loops and check-in points throughout the design process to hold themselves and the core team accountable for keeping these priorities in the forefront of their minds as decisions were being made about the design. The Veterinary Medicine Addition's A/E team used a 
crosscutting matrix (see Figure 10) to help participants understand how the design concepts intersected and complemented each other to optimize outcomes. To facilitate the process at the initial design charette they had several poster-sized displays, gave each participant three yellow sticker dots, and had each participant place the dots on the design concepts or intersections that each member felt should be prioritized. The $\mathrm{A} / \mathrm{E}$ team also asked participants to offer suggestions for how these constructs could be incorporated in the process and manifested as an attribute in the project.

Figure 10

Veterinary Medicine Addition's Design Principle Charette

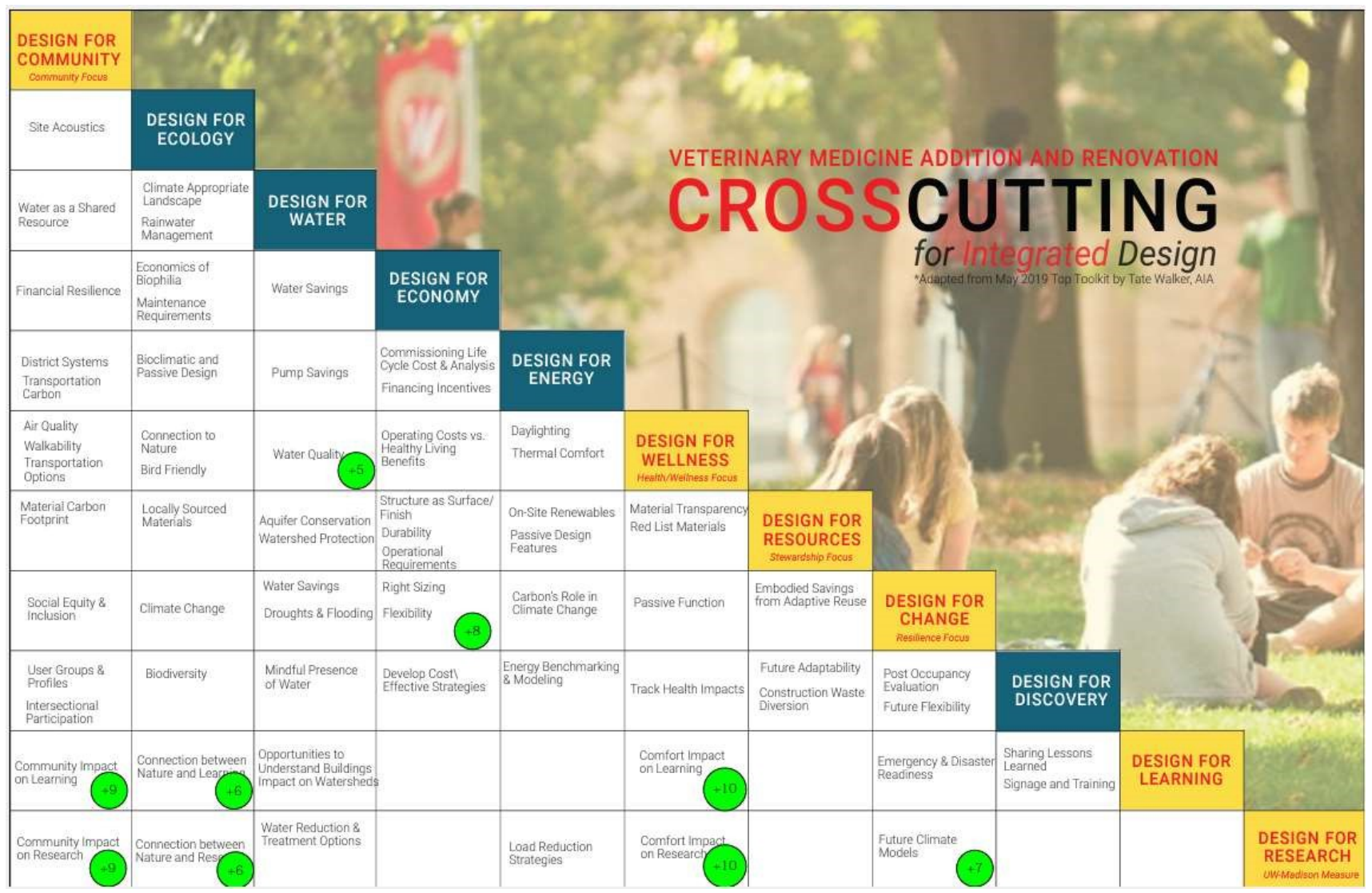


Note. The charette for this project included stakeholder integration and consultation to gather feedback and ideas on which design principles were a priority, and how they could be manifested in the building.

These preliminary design conversations at the very beginning of each project inform the remainder of the project process and are critical to setting the tone and values that the designers then incorporate in the construction documents for the building and landscaping. After these preliminary design meetings, the $\mathrm{A} / \mathrm{E}$ firm begins to bring the concepts to life in graphic designs, models, and digital mock-ups of the buildings. It is imperative that these concepts are deeply embedded in the project charter and design principles so that they don't get lost in the very dynamic back and forth among the hundreds of stakeholders that are brought in at various stages of the design process. The Veterinary Medicine Addition A/E team's method of participatory engagement in setting the design priorities, and intentionally keeping the priorities at the forefront throughout the process, ensured a persistence of these values which carried through the final construction documents.

The processes were so different for each of the projects that I was compelled to follow-up with the School of Veterinary Medicine and Division of Recreation and Wellbeing's representatives. The Recreation and Wellbeing team had a high focus on physical wellness, and the accommodation of gender diversity. Design principles as they were presented were not a priority for them, and they deferred to the expertise of the building professionals, especially when the conversations were technical. The School of Veterinary Medicine however, had a compelling story as to why their approach was so different. 
It was highlighted to me that the School of Veterinary Medicine already had a culture in which learning, wellbeing, and equity were prioritized and practiced. Veterinarians, and veterinary students work and learn in a high stress field, and the prevalence of suicide among veterinarians is high (Skipper \& Williams, 2012). While the attempt rate is comparable to the general population, the completion rate is higher given their access to lethal pharmaceuticals (Tomasi et. al., 2019). The School of Veterinary Medicine had been prioritizing these design attributes for a long time, it was part of their culture. While I had framed it differently in the design charette, the School's representative easily identified that these principles were already embedded in their practice. There was an imperative to not only incorporate elements in the building, such as areas of respite, but to teach self-care as part of the educational outcomes. As I was talking to the School's representative, she shared that one of their faculty had committed suicide just the month prior (K. Thorson, personal communication, April 2021). It was an incredibly poignant and humbling moment, and it highlighted the value of culture and community with incredible clarity.

The School of Veterinary Medicine had adopted and integrated practices that could have been meaningfully integrated at Recreation and Wellbeing but engaging the expertise and experience on campus is not part of the formal process. Generally, outside consultants or subcontractors are hired to advise the project's particular needs. However, the breadth and diversity of expertise at an R1 institution is inevitably going to intersect with the projects in different ways. In the case of designing for mobility considerations at the GymnasiumNatatorium Replacement, the faculty and clinicians were able to participate more actively in the design of their space. Similarly, at the Veterinary Medicine Addition project, the faculty and 
clinicians were able to advise on the floor finishes that were going to be best for animals to traverse. This has interesting implications for projects and advantages some projects more than others. For example, Germanic literature faculty may not be able to contribute expertise in the design of the building but could provide input into an aesthetic that compliments their area of scholarship. Whereas, when the mechanical and electrical systems for the School of Engineering are being designed, the engineering faculty may be too involved. Typically, however, the core team is informed by ongoing stakeholder engagement and, with some scheduled reviews and approvals of the Design Review Board, ultimately makes the final decisions throughout the design process.

\section{Integration Pilot 1 and 2: Evaluation}

These initial pilots provided incredibly valuable information which helped to inform the State of Wisconsin's Division of Facilities Development's adoption of a modified framework of these concepts. After completing the preliminary design pilots, the project manager for the State of Wisconsin (who served on both pilot projects) advanced the Design for Excellence Framework (formerly the AIA COTE Top Ten) method as a viable option to meet the exceptionally complex needs of the State of Wisconsin's building portfolio. The state's project manager leveraged long-established working connections among the various state agencies, gathered consultants from the AIA, as well as a few other select stakeholders, and worked through the complexities of developing a tool that is flexible enough to address the state's needs yet robust enough to meet the state's legislated requirements. These new guidelines were adopted by the State of Wisconsin and enacted September 1, 2020, less than 18 months after the first kick-off meetings for the two pilot projects. 
The flexibility inherent in the piloted design guidelines allows for an institution to adapt to the specific needs and priorities of the campus and the programs that the buildings and grounds are built to support. What I discovered in these pilot programs is that the integration of some of the concepts must be embedded earlier in the process so that they are included in the advanced studies. This will allow for them to be properly budgeted for in the enumeration, give bidders enough information to be competitive, and enable a more rigorous criteria to vet the $\mathrm{A} / \mathrm{E}$ firms that bid on the projects. Another very critical discovery was that performance metrics are tied to many aspects of the $\mathrm{A} / \mathrm{E}$ contracts and payments. Therefore, it is important to clearly define the expectations, especially for the design criteria that are qualitative and do not have established measures of success. These discoveries informed the second iteration of the process to incorporate design principles for learning, equity, and wellbeing.

\section{Integration Pilot 3a: Informal Learning Spaces}

The University of Wisconsin-Madison's Division of Information Technology has developed a Blended Learning Fellowship on Learning Spaces program that attempts to facilitate learning in, and outside of, the classroom. The program participants represent each of the campus' Schools, Colleges and Divisions, technology departments, facilities departments, and research centers as they work together to explore three focus areas each academic year (Blended Learning Fellowship, 2018-2019). As a result of my effort to integrate new design criteria for learning into the Capital Planning process, I was invited to participate in the Fellowship focus area tasked with studying informal learning spaces. 
The informal learning spaces group convened at the beginning of the 2018-2019 academic year, and I did not join until February of the Spring semester 2019. My participation in this study ran parallel to my applied work with the Capital Planning projects of the Veterinary Medicine Addition and the Gymnasium-Natatorium Replacement. At the time I joined, the Fellowship was in the middle of implementing a study of informal learning spaces and had placed a variety of furniture in two extended learning spaces (e. g. hallways and lounges). By the time I joined the Fellowship the assessment method had already been determined and the group was moving into the data gathering phase. I will note here that I built upon this assessment method for my proposal to evaluate the physical attributes of classroom space, another study that I will detail further along in this dissertation). In order to provide context and a baseline for the follow-up study I implemented, I will describe how the informal learning space furniture study was conducted.

Several pieces of furniture were procured or loaned from multiple manufacturers and were then placed in pre-determined areas of the Social Sciences and Van Vleck buildings. Signage explaining the pilot was placed throughout the halls, and people were invited to try out the furniture and provide feedback (see Figure 11). Scanning a QR code on the signage that was placed next to each unique piece of furniture (see Figure 12) prompted users to answer three questions. The first question prompted a four-point ordinal scale with values ranging from "It's worse than the floor" to "I'd come back just to use this furniture." The second question provided a five-point nominal scale inviting the user to indicate what activity was conducted while using the furniture and included options such as working with others or taking a nap. The third question was an open field for comments. Some of the comments gathered from the first week 
of the pilot were hilarious, for example one respondent stated, "I love this chair more than my own mother." Most of the comments described the function and comfort of the furniture, but there was one comment I found to be particularly poignant. A student noted that the furniture should remain "...unless it raises my tuition, then the floor is fine." It was a good reminder of the importance of being good financial stewards while we are improving spaces for our students. This tied directly with the purpose of my applied study, ensuring that the campus optimizes its resources for its mission.

\section{Figure 11}

Signage for Informal Spaces Study

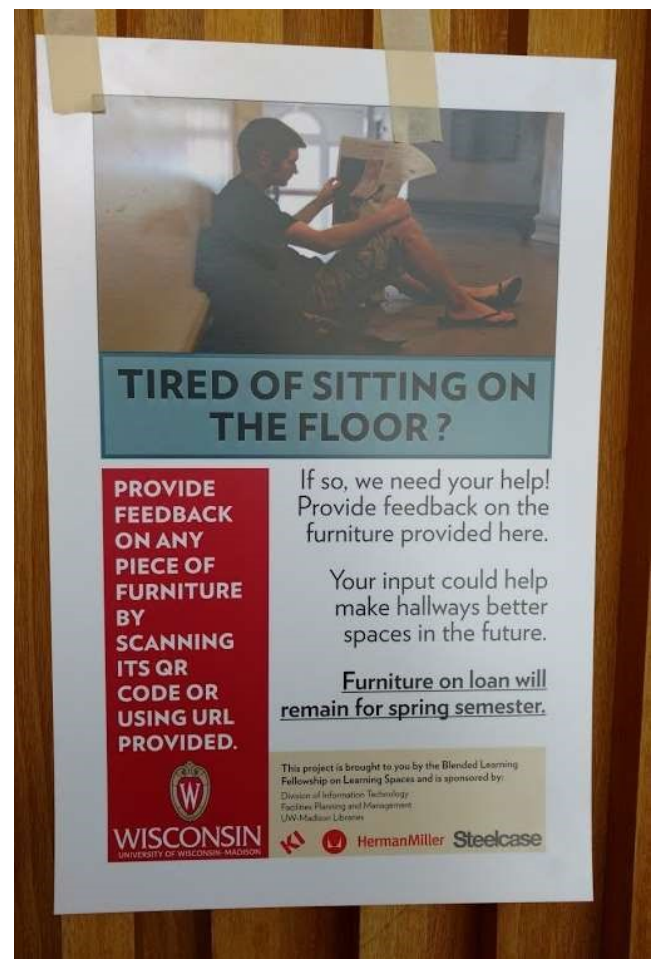


Note. The informal learning spaces study invited participation and feedback through direct signage explaining the study. This also allowed for sponsorship information encouraging business partner participation (own photo).

Figure 12

QR Code Survey for Each Piece of Test Furniture

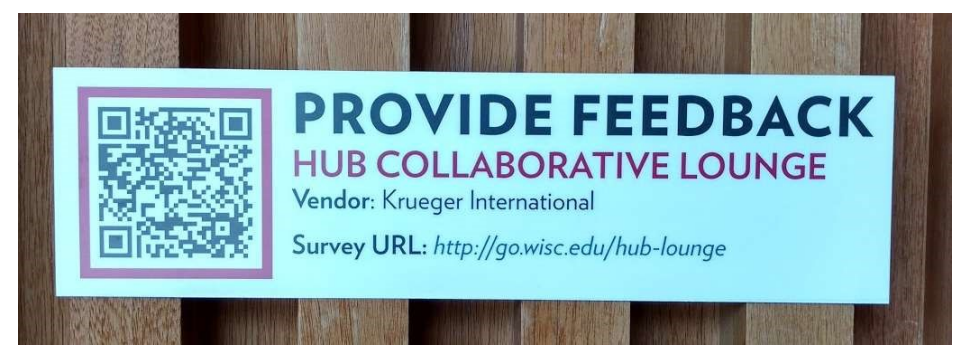

Note. Survey signage also allowed for vendor recognition (own photo).

The second part of the assessment was observational. The Fellowship members signed up for time allotments over the course of two weeks (the week before spring break and the week after spring break) to observe usage patterns. Of note, is that the physical attributes of the space are not included in either the survey or the observation. This is something that was discussed in a review meeting as an observer mentioned that the limited use of a particular piece of furniture may be due to the low lighting where the furniture had been place (see Figure 13). This is a critical piece of information because it provides an example of the impact that the physical attributes of a space can have on the functions that take place within that space. 
Figure 13

Example of Seating Placed in Informal Spaces
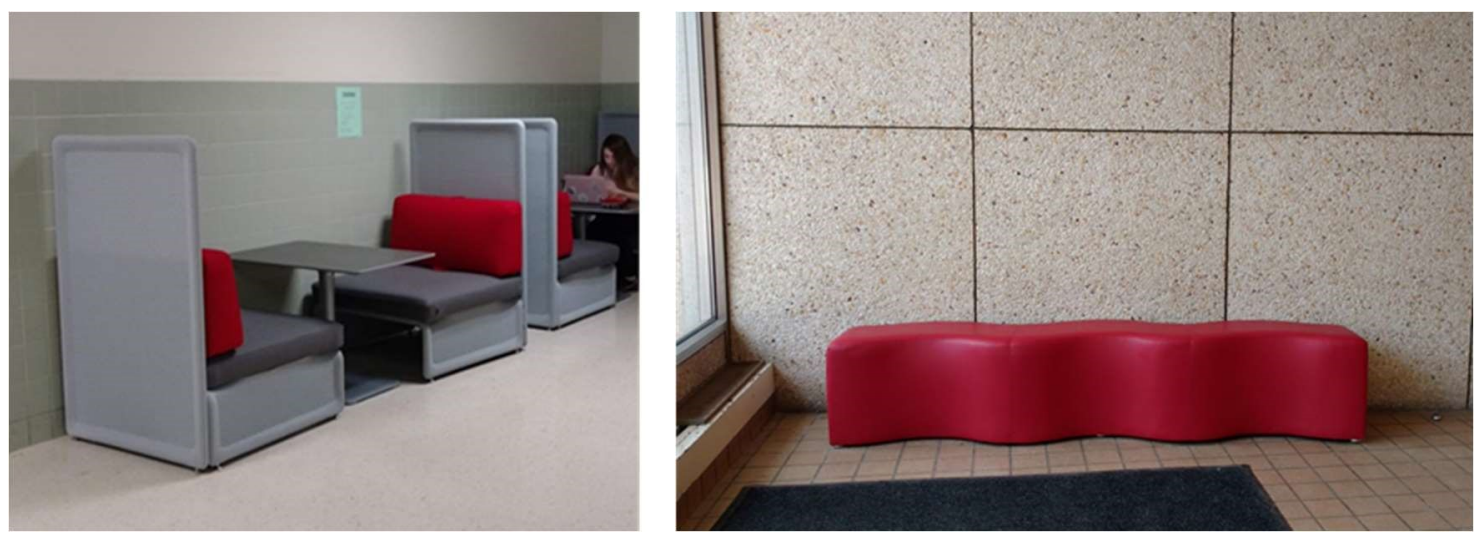

Note. Space attributes such as lighting were not included in the furniture use assessment (own photos.)

The dialogue among the Fellowship team resulted in several adjustments to the assessment tool and process, and those informed the follow-up study that I developed for the 2019-2020 Blended Learning Fellowship. Examples of issues that were addressed included: not being able to find where the furniture was placed, improperly installed furniture (a unique piece that was installed on its side), confusion about existing versus pilot furniture, and furniture missing altogether because it was back ordered. The issues were minor and were easily resolved, but they did cause some delay as well as inaccurate or incomplete information from the observations. The information gathered through observation, particularly the information not captured in the forms, was very valuable and demonstrated the importance of informal organizational structures and relationships since many of the observations were contributed from other building users who volunteered their own observations to the study team. 
Leveraging the transdisciplinary approach of the Blended Learning Fellowship was the ideal way to approach the assessment of general use classroom spaces. General use spaces accommodate teaching methods that are not specific to any one discipline, unlike laboratory spaces which can differ significantly among disciplines. A benefit of working with a similarly comprised team is not only their breadth of knowledge and experience, but their unique perspectives and personal passion for education. The composition of the team is made up of individuals, but the assessment methods also called for some crowdsourcing of observations. For example, in the pilot areas that were studied, building tenants (faculty and staff) were made aware of the program and invited to submit observations through an on-line portal. These observations and comments were informative and provided valuable information on how the surveys and observations can be improved, as well as how education regarding the study should be disseminated. For example, one building occupant noted that there should be no furniture in the hallway because of the fire code. This was an inaccurate in these pilot locations, therefore we learned that it is important to provide relevant information regarding adherence to codes and policies, and to relay that the pilot programs have all required approvals. It was also helpful to involve multiple constituencies, not only to ensure that all the details were properly addressed, but to raise awareness of the purpose of the study.

\section{Integration Pilot 3b: Designing Classrooms for Learning, Wellbeing, and Equity}

Following the study on informal spaces, I developed another Blended Learning Fellowship to design and implement classroom remodels focused on learning, wellbeing, and equity. This fellowship was comprised of ten faculty from different disciplines, an Interior Design specialist from Facilities, a student, a data analyst, and an accommodation services 
specialist. The Fellowship took place over the course of the 2019-2020 academic year. During the fall semester the Fellows were split into four teams and assigned a classroom(s) that was going to be remodeled. Each team was responsible for selecting the design attributes that would contribute to learning, wellbeing, and equity. In the Spring semester, over Spring Break, the classrooms were remodeled to their specifications. Only the faculty and instructors were made aware of the study. Assessments of the spaces were given to students in each of the respective classrooms at the beginning of the semester, and the intent was to conduct the same assessment two weeks after Spring Break. The COVID pandemic disrupted the second assessment, so for the purposes of this dissertation, I will focus on the integrated design process that the Fellowship undertook in the Fall 2019 semester.

In this study the research team are experts in their respective disciplines but are also students in organizational systems approaches. Three of the members (the Facilities staff member and two of the faculty) were experts in interior design, but no one was a specialist in designing for learning, wellbeing, and equity. Bringing their expertise together to design spaces for attributes required an iterative approach to accommodate the different methods and processes by which each member of the team engaged in the project.

These engagements had to be synergized to produce an applied outcome - the identification of specifications for materials for the rooms. Additionally, the survey instrument, which was developed in consultation with an assessment professional, had to be agreed upon by the Fellows. The synergistic transdisciplinary design allowed for adaptation of methods to enable the outcomes. 
Transdisciplinary research design methods are not prescriptive, Tashakkori and Teddlie refer to this quote from Greene (2007) which describes transdisciplinary research design methods as "an artful crafting of the kind of mix that will best fulfill the intended purposes for mixing within the practical resources and contexts at hand" (as cited in Tashakkori \& Teddlie, 2010, p. 24). The research design for this project was synergistic, which allowed for the combination of typologies, an iterative process, and addressed the "necessity of collaboration among researchers" (Tashakkori \& Teddlie, 2010, p. 321). Because each team was comprised of different subject matter experts and had different attributes of the spaces to design for, each team had a unique blend of inputs and outcomes.

The classrooms were selected by the Facilities department through an annual assessment process which inventoried and rated each of the classrooms on campus prioritized by use and selected based on a formulation that is used to weigh an appropriate portfolio mix of general use space which includes geographic neighborhoods of the campus. Basically, Facilities staff use a formula to ensure that a proportional number of general use spaces are remodeled throughout campus each year and allotted five of them for this study. The budget for the remodels was determined by Facilities, and they allowed the Fellowship a great deal of latitude and contingency with the budgets for the purposes of this research project.

The Fellowship met every Friday for the Fall 2019 semester. The Fellows split into four teams, and each selected a room to design. One team took responsibility for two nearly identical rooms in the same building. Through the course of the semester the Fellows learned about the various UW-Madison, UW-System, and State of Wisconsin processes and regulations that govern building and space design. Then they conducted research on the attributes within their 
rooms that were part of the remodel, and the impacts of those attributes on learning, wellbeing, and equity. They then applied the research to the selection of attributes that were available through state approved contractors, and that met state building codes as well as UW-Madison and UW-System standards. Their selections were then ordered by the Facilities staff with enough lead time to have materials on hand for Spring Break, and the Facilities teams were scheduled to accomplish all the work in that one week.

As a participant-observer, I worked with a facilitator to design the class for each week, and he facilitated the meetings. I brought in guest speakers such as the University Architect, the Facilities disability accommodation specialist, and a State Project Manager. I also provided available research from disciplines that were not represented, as well as asked each of them to bring their relevant scholarship and expertise to the selection process. I watched as the Fellows worked together, asked them to copy me on all e-mail correspondence, and collected their recommendations for their rooms and their accompanying notes.

The survey instrument for the students was developed as a draft and presented to the Fellows for their edits and revisions. Their suggestions were discussed and debated, and the edits that received consensus were included in the final version of the survey which was administered through a Qualtrics survey e-mailed to each student in each of the classes in each of the classrooms. The surveys were anonymous except for the room that they are attributed to. The survey addressed three aspects of the spaces (function, appeal, and physical comfort) and asked the students to rate the spaces on these three aspects as they relate to the attributes of the space. The faculty and instructional staff that used the space were to be randomly sampled to 
participate in an interview with a member of the Fellowship, but due to the pandemic that portion of the assessment did not happen.

This project used the design of classroom space to test a theory of systems approach in organizational functions. The design considerations intentionally forced an awareness of the environment's unintended impacts to physical health, wellbeing, comfort, accessibility, cognition, sense of security and sense of belonging. While the intent of a systems approach is to mitigate these unintended impacts, it was important to ensure that representation from the university's accommodation services team was a part of the research team and that these considerations were prioritized in the selection of materials. There was an expectation that the team would be a high performing team. Many of them had worked together before, and historically the applicants for the Fellowship have been people that value collaboration and have a genuine desire to learn. While I originally designed the study to examine how classroom spaces intentionally designed for learning, wellbeing, and equity impacted students, I was also able to use the study to examine an integrated design approach.

Of particular interest to my dissertation research was how this integrated design process worked across highly specialized knowledge sets. While it should be acknowledged that the participants in this Fellowship were likely to have a greater propensity for collaboration and be less wedded to the culturally embedded processes of Facilities personnel, there were Facilities specialists involved and there was a cross-pollination of ideas and learning among all the members. It was useful for the faculty to understand the complexity and limitations of the vertical hierarchy in which the university is embedded, it was useful for the Facilities staff to learn how pedagogies have to be adapted to accommodate the space, and it was useful for 
everyone to hear the lone student express the challenges the classroom space creates for his learning.

This part of the Integration Pilot, even though it did not turn out as expected, offered several very valuable insights which informed the next phase of this applied study, the analysis of campus processes for solving accommodation requests for lighting.

\section{Integration Study 4: Design for Equity, Lighting and Accommodations}

At the end of each semester students are invited to evaluate the courses they have taken. Questions can survey the relevancy of the content as it relates to the learning outcomes, the effectiveness of the professor's teaching methods, or the opportunities to practice what they have learned. The physical space of the classroom, and the extended space throughout the institution, are not assessed for their contributions to, or detractions from, learning. As has been discussed, campus spaces are the responsibility of the Facilities department, and at the University of Wisconsin-Madison the main campus includes over 400 buildings encompassing approximately 27 million square feet on over 900 acres. Faculty and staff have limited opportunities to provide input into campus design to accommodate their pedagogical methods, classroom, or office spaces. Students have virtually no voice in the campus design process which dictates the spaces in which they reside, study, eat and recreate. Given the sheer magnitude of the university's size and complexity of the environments it seems reasonable, in terms of efficiency and expertise, that one organization should be responsible for all of it

Yet one key take-away that I observed through the various integration pilot studies is the lack of the application of scalability. When Facilities is responsible for everything, the organization is inclined to uniformity - the organization sets standards to ensure that everything 
is as alike as possible for ease of maintenance, cost considerations, staff training, and product management. This directly reflects what the Facilities department is measured on - cost, facilities conditions, and maintenance backlogs. The observations and findings discovered through the parallel pilot studies repeatedly highlight the disconnect between the environmental needs of the occupants of the spaces, and the needs of the bureaucracy writ large. Throughout all of the concurrent, yet parallel studies, I applied the process mapping methods as an analytical tool. At the very high levels of the processes, it is the process itself that is governed by the bureaucracy. As the process maps become more granular, there is additional clarity and a growing recognition that despite the organizational push for the efficiencies provided by uniformity - humans will adapt their environments to suit their needs, and these adaptations cause disruptions that cause increasing ripples across the organization. In order to examine this adaptation phenomena, it was necessary to study an attribute that is present throughout campus, has variability among individual preferences, and has significant impacts for equity, learning and wellbeing.

A classroom space has physical attributes that include light, sound, air, temperature, texture, seats, workspace, color, technology, and 46 other attributes that, at the University of Wisconsin-Madison are tracked by the institution's Space Management Office (University of Wisconsin-Madison, 2012). The study of how humans learn is a critical component of research in education (Sousa, 2011). Ironically, while this research is predominantly conducted by researchers and faculty within institutions of higher education, it is only in the last decade that the application of this research is being considered in the design and construction of spaces at colleges and universities (Wieman, 2014; Freeman et al., 2014). At the University of Wisconsin- 
Madison, the prevalence and use of this research by competing institutions led to the development of an Ad Hoc Committee on Learning Space Improvement. The committee, formed in 2015, was tasked with the development of a "learning space improvement plan" (University of Wisconsin-Madison, 2016). The committee's final report noted the lack of viable assessment tools for learning spaces, and instead relied on recommendations based on feedback from stakeholders as well as current research on learning processes to inform its findings.

The findings of the committee resulted in a priority list of classroom space improvements predominantly focused on technology enhancements and the development of flexible spaces. These focus areas address the inherent need to improve technology access in classrooms, as well as to incorporate research on social interaction, as key components of learning (National Research Council, 2000). The priority list was enacted over a two-year period, utilizing a designated pool of funds exceeding \$2 million (which is what allowed the five classrooms to be available to the Blended Learning Fellowship for design). There were three considerations that could have enhanced the classroom space assessment effort and demonstrated that the investment in resources had been worthwhile.

First, there are several critical areas of learning theory that were not included in the assessment of the existing spaces. Cultural inclusivity, universal design and neurodiversity are just a few of the learning considerations that were not addressed. Second, the study did not expand into non-classroom spaces which, as was previously mentioned, is where most of the a student's time is spent. The campus itself is a learning laboratory, yet only the classroom spaces were included in the initial assessment. Finally, there is no indication from the Ad Hoc Committee's report that follow-up assessment of the spaces would be conducted to measure the 
effectiveness of the program or inform future iterations of the process. The assessment of a particular space (e. g. a classroom, a lounge, a coffee house) to ascertain its impact on learning can be done by evaluating the attributes of the space against the known impacts to learning. For example, research on lighting has identified impacts to productivity, sleep, security, mood, and biological cycles - all of which can contribute to, or detract from, learning. Lighting, as an attribute that impacts learning, wellbeing, and equity; met the criteria for the next study. The question then became how to scale the results of the assessment of individual and unique spaces to an entire university comprised of thousands of unique spaces with varying functions and light requirements.

As noted in the literature review, the impacts of lighting have demonstrated physiological and biological imperatives for all life forms, and on a college campus lighting is a consideration for security, safety, learning, sleep, wildlife corridors, energy consumption, and even research. It is the Facilities staff that determine the lighting standards for campus, with input from the University Police and the State of Wisconsin. There are many attributes of lighting that must considered, and in many applications, those are also prescribed. For example, in classroom settings there is a specific amount and color of light that must reach a surface. The energy efficiency of lighting is also a paramount consideration in campus design standards and has been the primary factor in the development of the lighting standards.

Facilities and environmental infrastructure account for approximately $7 \%$ of the directed financial resources of the institution ("Budget in Brief," 2018). Additional resources are designated by each School, College, Division, and Auxiliary (e.g., Student Unions and Athletics), as well as the State of Wisconsin, for capital projects and utilities. Instructional 
technology, disability concerns, student health services, student counseling services, and equity offices also all have budgets and staffs to serve the many needs of the campus. These combined investments account for a significant amount of financial and staff resources directed toward the built environment and mitigating the effects of the built environment. Suskie (2018) notes that Stewardship and Accountability are compelling reasons to assess the impact of this investment.

For this study of lighting as an equity attribute, I will reveal the solution first and then expound on the problem and the process that got me to the solution. Through collaboration between a willing School, and a student run Green Fund, the UW-Madison is undertaking a small project to remodel a general use classroom to serve as a demonstration and test model for equity in lighting. The intention is to provide a space for accommodation services to test different features of a room with clients to help identify how to provide the most suitable accommodations in the campus environment. The room will serve as a series of prompts in the mitigation discovery process. Flexible features such as tunable lighting, varied dimensional furniture, flooring, acoustics, natural lighting, and surface finishes, can help clients and the accommodation coordinators understand how the physical space affects the occupants. Accommodation coordinators will no longer have to guess at remedies while conducting trial and error experiments across campus throughout the semester. They will be able to solve a problem, prevent the disruption that arose from multiple process conflicts, and shorten the time needed to put effective accommodations into place without disrupting the other occupants or singling out the client as having need for an accommodation.

There are several additional benefits of the demonstration classroom which will help to provide an almost continuous feedback loop to inform the process of campus standards. The 
data gathered from this room, and similar test spaces across campus, will help the institution identify attributes of the room that are most amenable to the occupants. Through observation, interviews, and requests for particular pieces of furniture or lighting levels, data is gathered that informs future designs and campus standards so that inclusive spaces and equity by design reflect the campus' design as centered on learning, wellbeing, and equity.

In contrast to a mission focused design process, the current process to address a student accommodation is largely conducted by just a few positions on campus to limit privacy breaches, and to provide for fast and responsive service. The process begins when the Accommodation Officers receive the accommodation and the student's schedule. The student may meet with the officer to conduct a site visit or decline to do so. Accommodation requests are often vague, so the disability officer will make a change to the space, and then the client will visit and let the disability officer know if the change was effective or not. This trial-and-error field exercise is time intensive and requires a quick response from both parties. With schedules changing each semester it is critical that there is expediency in addressing the deficiency in the learning environment, it is not appropriate to have a student wait two months for an accommodation. This is where the process, if it were to follow the normal facilities work order channels, would be wholly insufficient.

Lighting throughout campus creates significant amounts of work to accommodate students and staff who are adversely impacted by lighting. My experience in the Blended Learning Fellowships demonstrated that lighting was a complex, but highly impactful space attribute, and the accommodations staff repeatedly lamented the challenge of dealing with lighting to find solutions for their clients. Since all lighting design and maintenance processes go 
through Facilities, I scheduled a meeting with two of the three campus design teams to ascertain the process by which they incorporate known learning methods, and recent empirical research, into their lighting designs for campus spaces. As a note of relevance, the three design teams all work in the Facilities department, in the same building, on two different floors, and report to three different directors. Although their functions are the same, the scope and responsibilities are different, and their work does not overlap. The initial meeting was unproductive when it devolved into discussions of procurement requirements and some accusations about who was following them and who was not. I began to look at other options for a solution.

Facilities designers are in many ways slaves to the Campus Standards, which as noted earlier, are developed upstream of the Facilities designers. But the issue was not limited to the color and intensity of the light, it was also a process issue that resulted from attempted mitigation methods. Through the process improvement discovery process, this study of lighting wound up involving several constituencies on campus including the three campus design teams, campus facility managers (dedicated staff employed by the School, College or Division to oversee maintenance and service), classroom scheduling staff, electricians, custodians, environmental health and safety officers, accommodations staff, waste hauling, procurement, recycling, and instructional technology.

In practice, the process begins with a client accommodation request. For this example, the client is a student. The Accommodation coordinator obtains the student's schedule and visits each classroom to ascertain mitigation methods. One mitigation method is to de-lamp (remove the bulb) above where the student sits. This action is not communicated to anyone since Accommodation Services does not use the same work order system as Facilities. The custodial 
staff come in on $2^{\text {nd }}$ shift, note the lightbulb is out and replace it, as per their training. The mitigation fails. Another mitigation method used is a fabric covering that gets placed over the fixture to soften the lighting. These had compliance issues with some of the light types due to flammability. According to the Accommodation Services staff, a litany of mitigation methods has been tried, everything from bringing in floor lamps to having students wear wide-brimmed hats. The mitigation attempts continue throughout the semester, and then the student's schedule changes, and the process starts all over again. This is a condensed version of several linear processes that work in conflict.

The lighting process improvement study examined the processes from procurement through disposal, through a discovery process of talking to everyone that touched classroom lighting. The early issues that highlighted the need for a better process were prevalent throughout every step of the process. The solution, to create a test space to gather data and raise awareness, is just a first step to fixing an organizational system failure. The key finding from the lighting process study for this dissertation, was that even within Facilities there are separate and distinct siloes and cultures, and there are jurisdictional boundaries that limit the adoption of a systems approach.

\section{Integration Pilot 5: Aligning Project Priorities at the Institutional Level}

"Give me a check box and tell me what to do." - Construction Project Manager

Many of the studies ran concurrently for the first 18 months of this integrated design

initiative. The findings were able to be immediately applied due to the repetitive, and continuous, cycle of campus design. The final study, for the purposes of this dissertation, is a culmination of the findings applied to the 2021-2023 capital project planning cycle and to small 
projects for the 2021-2022 fiscal year. Throughout these studies the goal was to find the most impactful points in processes to integrate design concepts that prioritized learning, wellbeing, and equity. The key discoveries pointed to the need for consistent advocacy, education and awareness across all stakeholder groups, tangibility, and scalability. With these in mind, a second phase of the integration pilot was developed with the goals of testing integration points earlier in the process, formalizing the integration of stakeholders into the process, and creating "check-off" forms that provided some standard applications and metrics for a few attributes that directly impact learning, wellbeing, and equity.

I began with awareness and education. Since we were in a virtual world of meetings, I created several slide decks that catered to the audiences I was speaking with, and I presented the design principles at internal, public, and external meetings for several months. The twelve Sustainability Design Guidelines, with ten of the principles mandated by the state, were well received as constructs, but they needed further clarification. People were struggling with the social principles. In particular, the human aspect of design was superseded by the focus on environmental impacts and energy usage. When I would engage participants through dialogue about equivalent metric tons of carbon, or energy versus electricity consumption, it was apparent that people were also unable to contextualize this language and these technical metrics. Fortunately, the AIA Design for Excellence Framework provided a plethora of resources to help frame the conversation. I also prepared real-life examples to help people contextualize the concepts. 
While I continued to present and educate about the design principles, I also had an opportunity to implement a second iteration of integration with two new capital projects, a new building in the College of Engineering, and a new Computer, Data, Information Sciences Building. Prior to the kick-off meetings for these two buildings, I made sure to present to the Campus Planning Committee, the University of Wisconsin's shared governance committee that approves and vets the campus' plans and prioritizes the capital project requests before they go to the Chancellor. Also serving as a Chancellor appointee to the committee is the Dean of the College of Engineering. I wanted to be sure to get these constructs in front of him as much as possible to socialize the principles before we got into the preliminary design phase.

Concurrent to the awareness campaign, there was a group of staff from across the UWSystem schools developing a checklist for the design principles (they apply to all State of Wisconsin agencies, so all the universities have to incorporate them). The only two checklists that I had to develop were Design for Learning and Design for Research, since the University of Wisconsin-Madison was the only school to add these mission-centric principles to the State's guidelines. The checklist (available in the appendix) made the project managers and A/E firms very happy and helped to simplify the process. Although, they still caused some confusion since while they have some uniformity to them, not everything applies to every project. The checklists are also limiting in that they offer solutions but do not prompt conversation that would lead to innovation and holistic approaches. The checklists compartmentalize the design principles and limit a systems approach that would optimize the features of the buildings.

The first test of the second iteration of the integration pilot was at the kick-off meeting for the Computer Data Information Sciences building. The A/E firm for the project has won 
AIA Design for Excellence awards and were well versed in the design principles and how they could be manifested in the project. They were intrigued by the two additional design principles, and knowledgeable about the extant research on cognition, mental health, and wellbeing. This project is an exemplar of the benefits of working with private sector firms that utilize the research produced at universities. After the initial kick-off meeting, the firm set up a series of conversations with key stakeholders, including students, and utilized the design principles as priority elements for the discussions.

The second test was with the College of Engineering. For this project I was also a little bit late to the party. Although the project has yet to be enumerated (it was $19^{\text {th }}$ on the Capital Projects list for UW-System at the time of publication), it had been deep in advanced planning. The initial meeting with the College of Engineering was with the Dean and several of the Associate Deans, all from different engineering disciplines. I honestly had no idea there were so many engineering specialties, I learned a lot and a key discovery for me was that even engineers do not understand each other. The differences in the disciplines are substantial enough that they readily acknowledge “....well, I'm not an electrical engineer, so I can't speak to that.” The Engineering leaders were very engaged, and I left the initial meeting with two observations, which I will expound on.

The first has to do with academic tribes. On the College of Engineering website, they list their values. One of them is "Encouraging collaborative, multidisciplinary research and teaching." During our conversations, I talked about creating spaces for wellbeing and equity, and incorporating attributes into the environment that are inclusive and would not be intimidating to non-engineering students. A couple of the Associate Deans chimed in and 
commented on existing spaces and how these active learning spaces with observational windows were tucked away in the bowels of buildings and were not readily accessible for anyone to observe. The conversation moved on to collaboration spaces and welcoming environments, and then someone said, “Well, we don't want to make it too accessible, we don't want English majors just walking in." I cannot recall, who said it, and the conversation did not stop, but the comment struck me as a direct contradiction to the values espoused on their website. It was a clear indication that the culture of the College does not embrace or practice their values, because then the conversation moved on to how those types of spaces could be moved further into the complex, creating barriers to outsider use (my words).

The second observation had to do with wellbeing. The College of Engineering had a student suicide resulting from the hostile environment created by a faculty member. As a result, there was a significant shift in how the College approached student wellbeing. One of the attributes of wellbeing that I mentioned to the group was related to a recent study on birds and happiness. This study noted that seeing and hearing four or more birds a day caused an increase in happiness, and that this could be manifested through intentional placement of windows and attention to landscaping and natural areas. The study even monetized the impact that birds had on happiness (Methorst et. al., 2021). As I watched my monitor (this was a Zoom meeting), several heads immediately turned down and I could see that some of the meeting participants were writing this down. Because I have my own biases about engineers, I imagined they were writing a formula; $<4$ birds $=$ happiness. At that moment, I recognized that wellbeing, equity, and learning, must be manifested in many forms throughout campus to truly be an inclusive and welcoming environment, and that there needed to be a cross-pollination of practices and ideas to 
create unique environments and escape institutional conformity. This cannot be done building by building, it has to be an intentional approach at a level high enough in the organization to escape the vertical hierarchy, yet not so low that it gets stuck in the quagmire of the boundaries of disciplines and the jurisdictions of operations.

\section{Assessment and Iteration}

\section{Examining the Metrics and Indicators}

\section{"It's time to put human metrics ahead of building metrics." Steelcase}

One of the more challenging aspects of assessing the impact of facilities design in higher education is that the standard facilities metrics do not align with the standard metrics for assessment of educational and learning outcomes in higher education. In the simplest terms, the performance of the building is measured not in terms of impacts to human and educational programming, but in terms of the performance of the building. Ultimately, the key performance indicators for the built environment are disconnected from the population it serves and the mission of the institution. To examine this at a more granular level, it was necessary to compare the standard facilities metrics, against the standard higher education metrics. Table 1 provides a comparison of select key performance indicators for higher education, and for facilities, as distinctive market sectors. 
Table 1

Comparison of Key Performance Indicators for Education and Facilities

\begin{tabular}{lc}
\hline Higher Education R1Performance Metrics & Facilities Performance Metrics \\
\hline$\square$ Enrollment & $\square$ Utility Costs/Energy Use \\
$\square$ Student Retention Rate & $\square$ Building Condition \\
$\square$ Six-year Graduation Rates & $\square$ Operating and Capital Costs \\
$\square$ Pell Recipients & $\square$ Campus Cleanliness \\
$\square$ Student to Faculty Ratio & $\square$ Work Orders and Maintenance \\
$\square$ Graduation Rates & $\square$ Space Management \\
$\square$ Post-Graduation Outcomes & $\square$ Capital Projects \\
$\square$ Research Dollars & $\square$ Safety and Compliance \\
$\square$ Average Annual Costs & $\square$ Gross Square Feet \\
$\square$ Acceptance Rate & $\square$ Staffing Ratios
\end{tabular}

Note. Standard reporting higher education metrics identified through institutional reports and the United States Department of Education College Scorecard (Berglund, 2019; Department of Education, n.d.). Standard facilities performance metrics identified through APPA's Facilities Performance Index, and Facilities Condition Assessments (APPA, n.d.).

The standard metrics for facilities performance are applicable to the built environment in any market sector in any geographical region. Measurements of the physical space as manifested through energy performance, space utilization, age, accessibility, and structural integrity result in useful comparisons whether the building is a residential high rise, or a biological safety level 4 laboratory. Facilities professionals share a common language and there are industry-standard performance indicators, both of which make the adoption of building designations, like LEED, attractive. The bundling of various attributes of the built environment under a moniker like LEED has proven to be effective for translating the complexities of building design and 
operation into a marketable, readily identifiable brand of efficiency and forward-thinking. Like many other market sectors, higher education institutions have leveraged this brand visualization as a sole performance indicator. Rather than having to tout two-dozen building performance metrics, a simple designation such as a LEED-Gold Certified building captures the essence of those metrics in one pretty package, generally advertised with an expensive, donor-gifted, sign.

For example, the University of Michigan has 17 LEED-Certified projects, and the most recent addition of their newest project was lauded as a successful sustainability measure, exampled by a March 9, 2021, press release announcing "LSA Building addition certified as LEED Gold" (Fisher, 2021) and highlighting the environmental attributes of the building. The University of Wisconsin-Madison has 11 LEED-Certified buildings, the most recent of which was completed a decade ago. These are a point of pride for the universities and contribute to several higher education rating and ranking systems such as the Princeton Review Guide to Green Colleges (Princeton Review, n.d.), Sierra Club's Cool Schools (Sierra Club, 2019) and the Sustainability Tracking, Assessment and Rating System (AASHE, n.d.). However, such designations for buildings do not reflect the quality of the learning environment, only that they were designed and constructed to minimize the impact to the environment. As was noted in the literature review, there are numerous designations available for buildings, however none exist that explicitly incorporate metrics associated with learning performance.

\section{Metrics for Learning Performance}

Both the University of Michigan and the University of Wisconsin-Madison use the standard set of metrics and performance indicators for assessing buildings in higher education. 
The Facilities Condition Assessment (FCA) reports are an industry standard advocated for, and facilitated through, the professional organization for higher education facilities, APPA (Kaiser \& Klein, 2010). APPA identifies the primary purpose of the FCAs as a standardized way of measuring the condition and functionality of buildings, infrastructure, and sub systems," yet the organization notes that the scope of the audits is traditionally limited to "physical deficiencies and compliance deficiencies" (APPA Website, n.d.). There is a module of the assessment that combines a physical assessment with a functionality assessment which "provides a more complete view of existing facilities and input for decisions" (APPA Website, n.d.). The functionality assessment is an additional option for facilities personnel to examine how the space meets programmatic needs. It is noted however, that this type of comprehensive assessment, although noted in facilities literature, is "the exception, rather than the rule" (APPA Website, n.d.).

Because the functionality assessment protocol was prepared by APPA for its higher education facilities professionals, I was hopeful that it would provide a more substantive alignment with the mission of higher education, even if it is rarely applied in practice. I examined how the functionality criteria of the Facilities Condition Assessment metrics aligned to the Performance Based Outcomes that the University of Wisconsin-System requires of its institutions to see if there were any measures that could represent a more direct relationship between building performance and educational outcomes. Table 2 identifies the attributes of the built environment that APPA recommends, rating these through a qualitative method approach of interviewing "a group representative of a program or department" allowing the participants to rate the attributes of a particular space on a Likert Scale of 1-5 $(1=$ Optimum, $2=$ Adequate, $3=$ 
Fair, $4=$ Poor, and $5=$ Unsatisfactory) (APPA Website, n.d.). Table 3 is the performance-based funding outcomes legislated for all colleges in the University of Wisconsin-System.

Table 2

Baseline Functionality Assessment of Spaces for General Classrooms

\begin{tabular}{cl}
\hline Space Type & General Classrooms \\
\hline Characteristic & Baseline Functionality Criteria \\
\hline
\end{tabular}

Classroom configuration and the size and arrangement of student and

1. Functional adequacy instructional stations satisfies instructional requirements, and provides adequate sight lines.

2. Accessibility Spaces shall meet ADA standards wherever required to meet program accessibility requirements.

Floors shall be covered in an appropriate, easily cleaned material that will permit the room to be maintained in a neat and orderly condition.

3. Room finishes Walls and ceilings shall be finished in appropriate, easily cleaned materials. Color schemes and finish materials shall present a pleasing appearance conducive to teaching and learning.

Floor covering, wall surface, and ceiling materials shall have appropriate 4. Acoustics and sound sound absorption and reflective qualities, and insulation against outside control noise shall be sufficient to provide a teaching, learning, study, or work environment free of distracting noise levels.

Heating and cooling systems, together with adequate control systems, 5. Climate control shall be installed that will permit the maintenance of a comfortable teaching, learning, study, or work environment at all seasons of the year. The installed lighting system shall provide an adequate quality and level of lighting for the teaching, learning, study, or work environment, and

6. Lighting shall be provided with controls to vary or adjust the lighting level as required for specific needs. Appropriate classroom window coverings shall be provided to permit unimpaired use of $\mathrm{A} / \mathrm{V}$ or other teaching equipment.

Adequate electrical capacity and outlets shall be provided in the room to 7. Electrical service accommodate teaching equipment, laptop computers, office equipment, etc.

(Table Continues) 
(Table Continued)

8. Instructional support As required, classrooms shall be equipped to support instruction, including:

- Connectivity to campus data networks and the Internet

- Chalkboards, whiteboards, projection screens, or other teaching accessories

- A full range of audio-visual equipment

Classroom fixed seating, when installed, shall be ergonomically correct,

9. Furniture and fixtures maintainable, provided with adequate tablet arms or table space for notetaking, and shall provide an unobstructed view.

10. All office spaces shall have appropriate connectivity to campus data networks technology and the Internet.

11. An adequate amount of storage space for equipment and files appropriate to storage space the function shall be provided.

Table 3

State of Wisconsin's Outcomes-Based Metrics

\section{Grow and Ensure Student Access}

1. Wisconsin high school graduates enrolled as degree-seeking undergraduates

2. Pell-eligible students enrolled as undergraduate students

3. Underrepresented students enrolled as undergraduate students

4. Transfer students enrolled as undergraduates

\section{Improve and Excel at Student Progress} and Completion

1. Undergraduates who have achieved 30 credit hours

2. Undergraduates who have achieved 90 credit hours

3. Undergraduate degrees awarded

4. Post-baccalaureate degrees awarded

\section{Enhance Operational Efficiency and Effectiveness}

1. Core Expense Ratio

2. Average number of credit hours required for an undergraduate degree

3. Average student debt on baccalaureate graduation

4. Degrees awarded per 100 FTE students

\section{Expand Contributions to the Workforce}

1. Graduates in STEM disciplines

2. Graduates in health-related disciplines

3. Pell-eligible graduates

4. Research and public service expenditures 
The analysis revealed only one potential point of connection between the learning environment and educational outcomes, and that was in the "Core Expense Ratio" which addresses the priority to "Enhance Operational Efficiency and Effectiveness" (University of Wisconsin-System, n.d.). The formula for this metric is based on ratios of expenditures of academic units and support units, as determined by:

1. A baseline set of outcomes for each UW institution established using a three-year average for each metric based upon data from the three previous years.

2. Similarly, a current year average will be established using the same factors that established the baseline data.

3. The current year and baseline data will be totaled and compared to determine whether the institution improved, maintained, or declined in performance. Funds will be distributed based on each institution's increase or decrease in their proportion of the total outcomes for the System. (University of Wisconsin-System, n.d.

Unfortunately for this aspect of the research for this dissertation, the legislated Outcomes-Based Funding measures were not able to accounted for in the 2021-2023 biennial budget due to the pandemic, which did not allow for "sufficient time to fully implement this change" (University of Wisconsin-System, 2020, p. 10). The four measures that UW-System did report on were standard metrics for higher education:

1. Undergraduate Degrees (conferred)

2. Participation Rate - the percentage of Wisconsin high school graduates who enroll immediately after graduation 
3. Retention Rate - the rate at which new freshmen return to the same institution for the second year of study

4. Graduation Rate - rate at which new freshmen earn a bachelor's degree at the same institution within six years. (University of Wisconsin-System, 2020, p. 10).

While the comparison of the metrics did not provide a crosswalk solution for the relationships between the built environment, and learning, wellbeing, and equity, it did highlight a few cultural and organizational aspects of APPA, the professional facilities organization, that are important to this study since APPA plays such an important role in training and networking for facilities higher education staff across the nation. First, it is important to note that APPA's recommendations for the functionality assessment serve the purpose of identifying upgrades to the spaces and monetizing those to "calculate a project cost" to include in a Capital Projects request. The intent of this functionality assessment is not to demonstrate a correlation that would contribute to the reporting of educational outcomes or more explicitly associate the investment in facilities to learning, wellbeing or equity. APPA notes that:

The combined assessment findings can determine a policy decision for a building renovation or replacement by answering the critical questions: Is the facility suitable for its current use, or will it require major renovations and/or modernization upgrading? What is the actual cost of remodeling compared with a new building, and is relocation of programs to another building feasible and desirable? (APPA Website, Facilities Condition Assessment, n.d.). 
While this model has potential to address some of the gaps between facilities' practice and its purpose, its lack of adoption and use by facilities professionals is a limiting factor. I noted two things about this tool that informed this study.

The first, is that the specialization of facilities personnel limits holistic approaches, in a manner that is very similar to the specialization of academic disciplines. The second, is that the tool, and the guidance that APPA provides for its use, focuses on singular program usage for spaces and emphasize maintenance as much, or more, than teaching and learning. Additionally, APPA advises that the process of gathering information through the interviews can be "delicate" due to the challenge of distinguishing "justifiable needs identified by space users that are potential "real" capital projects versus preference for unrealistic goals." This language and approach emphasize the facilities' culture of prioritizing maintenance and building performance over the humans, flora and fauna, and programs the environment serves. The subjectivity of determining whether a request made by a student or faculty has merit or is "unrealistic" falls to the Facilities staff by default of the campus design process.

An example of this happened at Illinois State University during the renovation of Schroeder Hall in 2005. The A/E firm and facilities personnel allocated three shelves per faculty office in the remodel, more shelves were deemed to be excessive. The disciplines housed in Schroeder Hall are History, Sociology \& Anthropology, and Criminal Justice; all of which rely heavily on books. In fact, for some disciplines, particularly in the humanities, the publication of books is more critical to a scholar's reputation and body of work than the publication of articles. The approach taken by the Facilities Department to limit something as simple as shelving was anachronous to the mission of the institution and demonstrated a clear disconnect between the 
built environment, and the program and people it is intended to serve. Such disconnects are amplified when the spaces and the populations get more diverse.

The comparison of the standard industry metrics for facilities, and the standard higher educational sector metrics, returned no identifiable connections as to how the learning environment contributes to the educational outcomes. The analysis of the metrics did however enable a closer look at the culture of the professional organization for higher education facilities, APPA, as reflected in the training and resources they provide to facilities personnel. This information was very helpful in informing my study and helping to identify obstacles in facilities organizations that impede a holistic approach to campus design. Even though researchers have identified that increased performance is related to the work and learning environment, and APPA acknowledges this in the training provided to facilities professionals, there is no advocacy from APPA to make this a priority. In fact, the focus continues to be on building performance and maintenance. In light of this observation, I re-reviewed the studies I noted in the literature review and leveraged the Fellowship program to run the classroom design pilot study that demonstrated these concepts in a tangible manner.

\section{Facilities Departments and Professional Development}

"I've been designing and constructing buildings for over thirty years, and I don't feel like I know how to do it anymore." - University Architect

Facilities departments encompass a great deal of specialized expertise and experienced professionals. The professional organization for Facilities is called APPA: Leadership in 
Educational Facilities, and while the acronym is outdated (Association of Physical Plant Administrators), the organization has retained the acronym as its moniker. The organization has been around since 1914 and has evolved along with the institutional facilities professionals that it serves. APPA states it purpose as threefold:

- APPA transforms individual facilities professionals into higher performing managers and leaders, which...

- Helps transform member institutions into more inviting and supportive learning environments, which...

- Elevates the recognition and value of educational facilities and their direct impact on the recruitment and retention of students, faculty, and staff. (APPA, n.d.)

I want to emphasize that two of the three purpose statements deal directly with learning and highlight the importance of "educational facilities."

APPA is an organization that supports the professional development of its members, and one of the ways it does that is through a Body of Knowledge (BOK) which they use to certify and educate facilities professionals. Table 4 provides the content areas of APPA's Body of Knowledge, and it is important to note that the content contains no information about higher education organizations and cultures, and no information about how the facilities environment contributes to education and learning, or impacts the recruitment and retention of students, faculty, and staff. Granted, there is implicit understanding that cleanliness and good air exchange rates contribute to health, but there is no direct and targeted content to help facilities professionals truly understand and create educational environments. 
Table 4

Curricula for APPA's Body of Knowledge (BOK)

\begin{tabular}{|l|l|}
\hline Part I: General Administration and & Part II: Operations and Maintenance \\
Management & - Building Architectural and Structural \\
- Budget Recharge, Cost Recover, and & Systems \\
- Rates & - Building Control Systems \\
- Change Management & - Building Electrical Systems \\
- Communication & - Building Fire Protection \\
- Customer Service & - Building Interiors \\
- Effective Talent Management & - Campus Security \\
- Financial Analysis and Control & - Capital Renewal and Deferred \\
- Leadership & Maintenance \\
- Managing Ethically & - Cleaning Operations \\
- Organization & - Elevator Systems \\
- Staff Development & - Emergency Preparedness and Business \\
- Strategic Role of Human Resources & - Continuity \\
- Strategy and Leadership & - Fnvironmental Health and Safety \\
- The Five Styles of Negotiation & - Facilities Condition Assessment \\
& - Fire Prevention on College and University \\
& - Campuses \\
& - Grounds Maintenance and Operations \\
& - Recapitalization Management \\
\hline
\end{tabular}

(Table Continues) 
(Table Continued)

\begin{tabular}{|c|c|}
\hline $\begin{array}{l}\text { Part III: Energy, Utilities, and Environmental } \\
\text { Stewardship } \\
\text { - } \quad \text { Campus Computerized Control and } \\
\text { Monitoring Systems } \\
\text { - Campus Utility Systems Master } \\
\text { Planning } \\
\text { - Central Heating Plants } \\
\text { - Colling Systems and Thermal Energy } \\
\text { - Storage } \\
\text { - Data and Voice Network Infrastructure } \\
\text { Domestic and Fire Protection Water } \\
\text { Supply and } \\
\text { - } \text { Eistribution Systems } \\
\text { - Energy General Distribution Systems } \\
\text { - Energy Management and Conservation } \\
\text { - Roadmap for Campus Environmental } \\
\text { - Sustainability } \\
\text { - Sanitary Sewers and Stormwater } \\
\text { - Management Systems } \\
\text { Source Energy }\end{array}$ & $\begin{array}{ll}\text { Part IV: Planning, Design, and Construction } \\
\text { - } & \text { Building Information Modeling } \\
\text { - } & \text { Campus Master Planning } \\
\text { - } & \text { Capital Budgeting } \\
\text { - } & \text { Design Managemention Management } \\
\text { - } & \text { In-House Design and Construction } \\
\text { - } & \text { Infrastructure Planning } \\
\text { - } & \text { Organization and Management of } \\
& \text { Capital Projects } \\
\text { - } & \text { Programming } \\
\text { - } & \text { Project Delivery } \\
\text { - } & \text { Real Estate } \\
\text { - } & \text { Renovations } \\
\text { - } & \text { Site Development } \\
\text { - } & \text { Space Planning and Administration } \\
\text { - Sustainable Design and Construction } \\
\text { - } \quad \text { The Building Commissioning Process } \\
& \text { Value Management }\end{array}$ \\
\hline
\end{tabular}

This lack of awareness and availability of training for Facilities staff was a significant obstacle for integration of design principles that are focused on societal and educational outcomes. Fortunately, there were several very strong advocates and professionals at the University who had come from sectors that had been early adopters of some of these principles, and their observations and guidance were critical in evaluating the initial design integration pilots and informing a second iteration. 


\section{Summary}

There were several key findings throughout the studies, many of which were able to be used to adjust the studies almost immediately. However, I was most interested in the findings that could potentially enact organizational change with a greater impact across large portions of the University. This proves to be very difficult given the organizational hierarchies and antagonistic politics in Wisconsin. There is a propensity to work against each other, in fact the system is set-up to create competition instead of collaboration, as exampled by the capital budgeting process in which each of the institutions is pitted against each other for resources. Despite the politics of the State, and the cumbersome nature of the organizational systems, the process continues to slog along, albeit ineffectively as is evidenced in UW-Madison's

plummeting rankings from a top five research institution to a steadily downward trajectory, and it being the only flagship institution in the country to have no financial mechanisms for selfsupport.

What I learned from these studies is that it is imperative to socialize the design principles in a way people can envision what it looks like. Diversity, Equity and Inclusion are not new constructs, what is new is the idea that these can be intentionally manifested in a place as a central design imperative. The virtual world that we have all lived in for 2020 has helped people to understand that construct, largely through their own experiences and being able to see into other people's homes. No one has a home office that looks like an institutional office. Even the video feeds from people's cars have shown more character and personality then our campus spaces. Experiential learning has been the biggest boost to this process and has helped to inform how to present these design principles. 
A second finding is the need to start earlier in the process, even before advanced planning. It is the stakeholders and building owners that need to prioritize the design principles, to advocate for them throughout the process. Every facilities condition assessment should include the impact to learning, equity, and wellbeing, which will then inform other assessments, and will become part of the advanced planning priorities naturally. This is a cultural component which cannot be dismissed. Stakeholders must be engaged throughout the process, at intermittent points of the sequence, and they must also serve as advocates to ensure persistence of the principles through construction.

A third, and final finding is the need to leverage and enable expertise across the adaptive organizational systems. This can be accomplished through a process of learning from each project to inform the next. This is particularly true for Deans. Most Deans will only get to build one building in their career and having a network of peers to learn from is invaluable. It is one thing to be advised by an A/E firm, or the Facilities professionals, it is quite another to hear from another Dean about what aspects of the buildings and grounds have been most beneficial, which are underutilized money pits, and what they would have done differently. We are educational institutions; we should leverage the knowledge that we have gained and construct our Campuses with it. 


\section{CHAPTER V: CREATING WALKWAYS IN THE SKY: BRIDGING THE SILOES \\ Introduction}

This study examined how higher education organizations design campus environments, and how to intentionally design for learning, wellbeing, and equity. There is a demonstrated correlation between the learning environment and positive learning outcomes, and the constructs of wellbeing and equity contribute to the mental and physical states that enable cognition (Berglund, 2019; Cash \& Twiford, 2010; Earthman, 2002; Schneider, 2002). Yet in practice the design of institutions is not centered on the creation of learning environments. It is a design process that claims that strategic objective, but the process is discordant, allowing for individual agencies within the institution to prioritize their programmatic needs ahead of a centralized institutional imperative. This disconnect between practice and purpose can be traced to the disparate organizational systems inherent in higher education. These are often referred to as the sides of the institution, the academic side, and the operational side. Even within these dissections of the organization there are cultural and organizational impediments to a holistic approach to create a learning environment (Bergquist \& Pawlak, 2008; Schein, 2017).

This study undertook an examination of the organizational systems that influence campus design to discover enabling or disabling components of the systems, how the impacts of the learning environment can be measured, and to identify the potential indicators of successfully creating a learning environment. The primary focus of the study was on processes as an artifact of systems interactions, controls, and adaptive mechanisms (Sharp, 2012). The institution that was studied was challenging because of its placement in a complex 
vertical organizational hierarchy. These additional organizational systems wield inconsistent and unpredictable influence over the institution, resulting in an extraordinary expenditure of human and financial resources to maintain the integrity and forward motion of the institution. The study methods had to be adapted often to be able to capture information and change direction when discoveries were made that disrupted the plan.

There were advantages to this environment. The repetitive nature of the campus planning and project development process allowed for robust applied research opportunities. The size of the institution and the sheer number of projects distributed throughout the process at any given time enabled multiple iterations of the studies and allowed for complimentary studies to be spun off and implemented quite easily. This allowed for the original study of the integration of design principles into two capital projects, to be expanded to several other studies that allowed me to test integration points at different stages of the design and project process. Given that this research was designed to implement a change to a process, and then analyze it for effectiveness, this institution proved to be a very productive study site.

\section{Summary of Findings}

Given the number of studies I was able to undertake, and the dynamic nature of the institution itself, there were a number of findings that I was able to use to inform second iterations of the studies. I will begin with the earliest finding that centered around the limitations of influencing change in the vertical hierarchy in the State of Wisconsin. The organizational systems limitations imposed by the legislated bureaucratic hierarchies are not insignificant. The University of Wisconsin-Madison expends precious time and resources trying to influence the decision makers in the vertical hierarchy, with limited success due to the competition that the 
state's process imposes on the UW-System Schools. The State of Wisconsin's Capital Budget process pits the schools against each other, limiting inter-institutional collaboration, diminishing opportunities to optimize curricular and research enterprises, and pushing its flagship institution further down in the national rankings. This has a deleterious effect on other revenue streams because the institution lacks the agility and flexibility it needs to respond to emerging markets and knowledge enterprises. This is evidenced in the decade long process needed to get a building completed, and that ten-year time frame is only if the building gets enumerated or is funded through donors. The fact that only 6 of the 128 projects advanced by the University of Wisconsin-Madison made it onto the UW-System capital project list is problematic for the institution's short, medium, and long-term planning (UW-System, n.d.). Additionally, the UWSystem is only the second step in the vertical hierarchy, the projects have to advance through four additional approval processes at the state level. This puts the institution at a significant disadvantage in its ability to gain students, recruit and retain faculty, garner philanthropic gifts, develop corporate partnerships, and compete for grants. For comparison, the University of Michigan has been able to leverage their time and energy into garnering over $\$ 1.6$ billion toward their learning environments, increasing their rankings, reputation, and revenue generation capacity (Allen, 2019).

Another limitation to an organizational systems approach is a cultural component that is evidenced in the disciplines (Becher \& Trowler, 2001; Bergquist \& Pawlak, 2008), and in the Facilities organizations (Wright \& Wilton, 2012). There is a lack of awareness and education around the impact of the environment on learning, and an internal competition for resources which encourages advancement of siloed agendas by independent actors, negating the holistic 
approach of organizational systems. Neither Facilities personnel, nor the academe as a whole, are skilled and educated about designing a learning environment. Even within the academe, the scholars that study learning, wellbeing, and equity are different than the scholars that study building design and landscape architecture. While there are pockets of scholars that do both, just as there are $\mathrm{A} / \mathrm{E}$ firms that are on the emerging edge of the practice, it is not yet common, and is an area that requires additional education and awareness.

Conversely, one of the areas that enables an organizational systems approach to campus design for learning, wellbeing, and equity is also culture. This is where the adaptive organizational systems prove to be incredibly advantageous, both internal and external to the institution. It was the adaptive organizational system that enabled the adoption of a new set of building standards for the State of Wisconsin. Building standards that include some human metrics, not just building metrics. The cultures of the academe are also inquisitive, constantly seeking new knowledge by building on the old, and while it may be human nature to resist change, it is the nature of scholarship to seek the new and undiscovered (Bergquist \& Pawlak, 2008; Brown, Harris \& Russel, 2010; Schein, 2017).

Another enabling feature of the process analysis, was the repetition and frequency of the project cycles. In project terms it is probably analogous to the life cycle of a fruit fly which allows for many cycles of research in a short span of time. While some of the projects may take decades to complete, the fact that there are hundreds of active projects all of the time, makes the cycle of integration advantageous for socializing and familiarizing people to the design principles. One of the biggest advantages to these design principles is that they allow for creativity and innovation, and they encourage bringing in new voices and perspectives to the 
table. The opportunities to incorporate new ideas and new mental models into the campus design process is very promising.

The expansion of the concept of learning spaces was also a positive and enabling outcome of these studies. While there are strong advocates for natural spaces on campus, the expansion of the concept of learning spaces into unprogrammed and underutilized spaces was quite refreshing. Something as simple as placing furniture in hallways was remarkably well received, especially in light of how much furniture gets sent to surplus each year. It was an extraordinary example of optimizing spaces for learning, wellbeing, and equity at no cost to the institution.

While the opportunities are vast, so are the challenges. Not necessarily the challenges to the design integration process at a small scale, but the challenges to operationalizing these changes at an institutional level. There is a very strong disconnect between how facilities are designed and maintained, and how the facilities are used. For example, in the March 2019 Design Review Board meeting on the new Parking Deck for the Veterinary Medicine Addition, the board members spent approximately 20 minutes discussing the color of the bricks, 25 minutes discussing the species of trees and the landscaping (which were deemed to be too many varieties, the wrong varieties, too close to the building, the wrong color, etc.), ten minutes discussing the glass stairwells and the window fritting to mitigate bird impacts, and zero minutes discussing the clients - the animals and their owners that will be parking there to visit the veterinary clinic.

Near the end of the public meeting, I slipped a note to the University Architect with bullet noted inquires on (a) the toxicity of the plants to animals, (b) the vulnerability of the 
landscaping - particularly vertical features - to urine, (c) placement of pet walking spaces and waste stations, (d) potential impact on paw pads from the temperature of the proposed road surfaces based on the colors and materials they had selected, (e) treatment of road surfaces for impact on paw pads and toxicity when animals lick their feet, and (f) surfaces and open stairs dogs' eyes are generally at knee level which means that open stairs, shiny surfaces and glass walls are difficult for dogs to see - this can cause panic or a reluctance to move, which could cause falls for their humans and injuries to themselves. The Design Review Board acknowledged these inquiries with promises to follow-up, and the University Architect asked the design firm (a different firm than the one building the Veterinary Medicine Addition) to include the Veterinary Medicine folks in all planning meetings. After the meeting two of the board members approached me to thank me, "on behalf of their dogs," for the inquiries.

This example demonstrates the communications and planning challenges that confront existing processes. The processes maintain silos that are differentiated by professional expertise. In this example the architects were wholly consumed by the aesthetics of a parking garage and gave no consideration to the fact that there will be animals walking through traffic and stepping in puddles of vehicle fluids, or that elderly people will be trying to navigate stairs or elevators with skittish pets. Or that dogs will be peeing everywhere, while puppies will be tasting and swallowing everything they can get their teeth on. Ensuring that the School of Veterinary medicine is more integrally involved in the design of their parking deck should help to alleviate some of those issues. However, the veterinarians are also not designers or engineers, so the teams need skilled facilitators that can respect and incorporate everyone's knowledge and expertise throughout the project. 
This is a significant challenge to overcome in an institution that prides itself on both shared governance and high levels of divisional autonomy. Inviting a more collaborative approach and leveraging the expertise on campus can enhance, delay, or destroy a project. In an institution with limited resources and tightly legislated controls on the mechanisms for financing, delay can often guarantee failure through outright collapse of a project, through dismal results due to obsolescence of a design or technology, or through escalating costs from the design phase through substantial completion and occupancy. The enablers and disablers in an organizational systems approach varied by project and participants, but the more exposure people had to the design principles the more they were inclined to incorporate them into the next projects. The adoption of the principles is one measure of success that was identified, and through each integration pilot additional metrics and performance indicators emerged, some more feasible than others. Ultimately, the tool that was adopted included the coveted checklists, as well as metrics for the suggested attributes. As in any good bureaucracy, these checklists also serve as reports which are included in the project documents and provide the institution with data sets that will be the source for the performance indicators.

The tool is a spreadsheet which enables linking for more robust data collection. It also includes worksheets for each of the design principles, and a comprehensive checkoff list that enables the core team to identify which principles and attributes are going to be included in each project. It also identifies which principles, attributes and metrics are required and by which level of the command-control hierarchy. Additionally, there is a listing of preferred attributes which provide project owners ideas and opportunities to tailor their projects to their program needs. An example of the checklist for Design for Equitable Communities is included in Table 5. 
Explanation of the metrics, required attributes, and additional resources are provided in each of the respective work sheet tabs.

\section{Table 5}

Design Summary Checklist for Equitable Communities

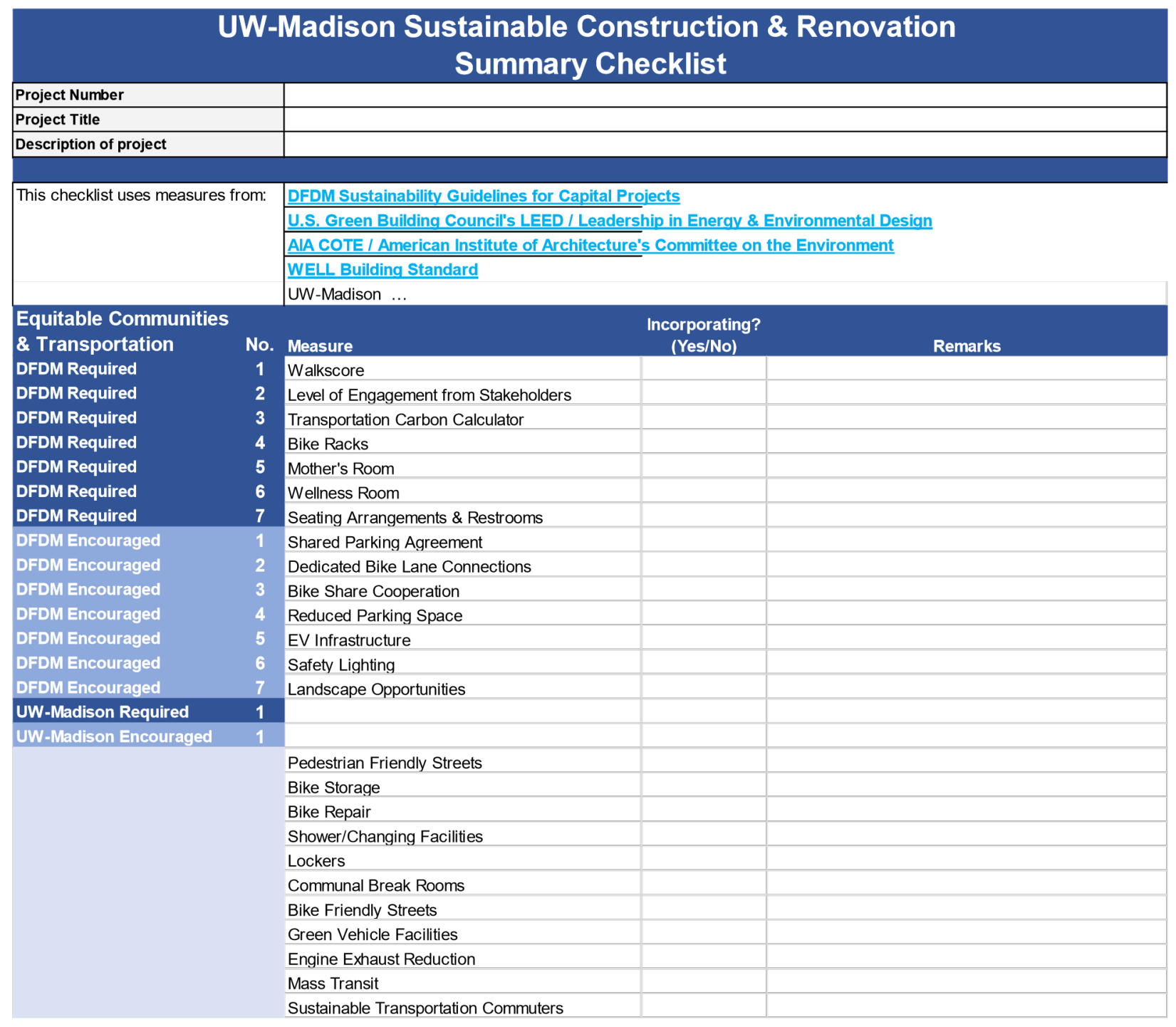


Diving a little deeper into the tool provides the additional information that the project

managers need. For example, the inclusion of a Mother's Room is determined based on building occupancy, and it is a binary decision - yes, it is required, or no, it is not required. If it is required, the attributes of the room are also identified in the worksheet tabs. The information for the Mother's Room is listed in Table 6.

Table 6

Example of Metrics and Attributes for a Mother's Room

Mother's Room

Required for or all New Construction/Major Renovation (greater than \$3 million): Address Walkscore, Transportation Carbon Calculator, and bike racks above.

\footnotetext{
(Table Continues)
}

Provide (1) mother's room per the first 200 occupants, and additional capacity for each additional 200 occupants, which may be additional mothers rooms or a design which provides multiple private areas for pumping/nursing and a shared washing and storage area. The mother's room shall meet the following minimum requirements (Reference WELL v2 C09 for a summary of the Issue and Impact):

- ADA accessible and including all components noted herein (typically requires approx. $7 \mathrm{ft} \times 7 \mathrm{ft}$ footprint)

- Single, dedicated function - e.g. not shared space with a Wellness Room

- Minimum single basin sink minimum 9 inch in width and length for hand washing and equipment washing including an adequate faucet, minimum 3 inches from any edge of sink with 10 inch minimum column of water d. Paper towels, soap, waste bin

- Chair and adjacent minimum 30 inches wide x 20 inches deep open below counter space, work surface, or tabletop for pump and bottles to rest on while in use, (2) minimum receptacles adjacent, ideally at counter height $\mathrm{f}$. Microwave optional yet beneficial for equipment sanitization

- If the space serves a work area with full-time employees (FTE), system in place

- for booking / scheduling designed to account for privacy, e.g. by using an occupant number in lieu of name if occupant prefers. 


\begin{tabular}{|l|l|}
\hline - & $\begin{array}{l}\text { A refrigerator with dedicated and sufficient space for milk } \\
\text { storage based on assessment of occupant storage need for } \\
\text { regular building occupants. }\end{array}$ \\
- & $\begin{array}{l}\text { Dedicated storage space for pumps and pumping supplies } \\
\text { (e.g. shelving, base cabinets, or lockers) }\end{array}$ \\
- & $\begin{array}{l}\text { If the space serves an area with FTE, consider additional shelf } \\
\text { or counter space for equipment drying. }\end{array}$ \\
- & $\begin{array}{l}\text { Door to pumping/nursing area that is lockable from the } \\
\text { interior with indication of 'in use' or similar message that is } \\
\text { visible from the exterior } \\
\text { Acoustic and visual privacy to adjacent occupied space. } \\
\text { While visual privacy is required, daylight and views are } \\
\text { beneficial, such as through translucent glazing or to an } \\
\text { exterior unoccupied area. If any transparent or translucent } \\
\text { glazing is present, user-controlled window coverings are } \\
\text { required }\end{array}$ \\
\hline
\end{tabular}

There was also a requirement initiated that the design principles be written into the project charter so that they are not lost through the project process. This was one way to ensure that even without a consistent advocate that they would persist. Adoption of the principles, and persistence through the project are the performance indicators. There is now also the ability to focus in on the design principles as a lever for the institution to push out a central priority. For example, if the adoption and persistence of Wellbeing as a design principle is lagging, the institution can opt to mandate options within Design for Wellbeing for all projects.

One of the more positive outcomes of the State's adoption of the design principles is that projects that include them will be more favorably ranked in the capital budgeting process, so it should advantage projects throughout the vertical hierarchy and arduous approval process. Since all three levels of project managers must also report on the design principles there is additional oversight at every level of the command-control system. Ultimately, it was the adaptive 
organizational system that has been most enabling for these integration studies. The commandcontrol system, while necessary to some degree, is very limiting, especially in Wisconsin. Which is good news for the process of designing for learning, equity, and wellbeing, since it did work at the University of Wisconsin-Madison despite the limitations, it could have significant implications for application elsewhere.

\section{Implications in Practice}

Systems thinking can be challenging to apply in practice, and as a result it can become an exercise in scholarship untethered from the practical realities of organizational systems. I have personally found that it is difficult to effectively communicate the methodology and benefits to Facilities personnel in particular, in large part because the interconnections and dependencies are without boundaries. In Facilities organizations, projects and work are generally delineated within defined scopes and jurisdictional responsibilities. There are processes in place to identify and track any change in the scope of a project (e.g., change orders), or any work that crosses jurisdictional boundaries (e.g., contract language or mediation services), and these tools are useful to keep processes confined into narrow lanes and to achieve quantifiable and actionable workflows. They also limit innovation, collaboration, and connection to the university's mission hence, I use B.O.B. as a model for visualization of the concepts.

Benzoate Ostylezene Bicarbonate, also known as B.O.B., is an adorably dimwitted character in the movie Monsters vs. Aliens. B.O.B., is a blue, one-eyed, indestructible, gelatinous mass with no brain, yet an uncanny sense of comedic timing. The beautiful thing about B.O.B., beyond the fact that he is the by-product of multiple questionable food science 
experiments, is that he is a useful visual construct to represent the breakdown of linear processes when mapping a systems approach (see Figure 14).

Figure 14

Graphic Visualizations and Representations of Systems Approaches
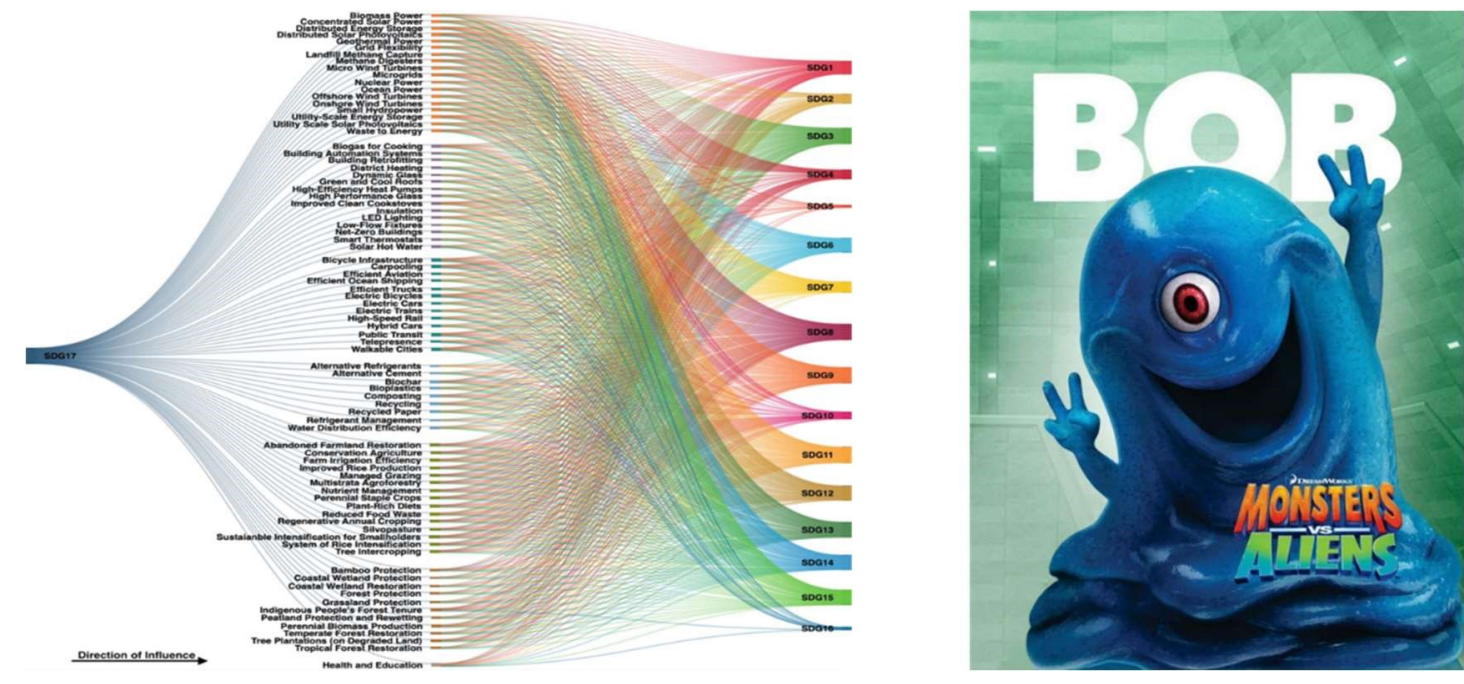

Note. The figure on the left is a representation of how the Sustainable Development Goals are mapped to the climate change solutions in Project Drawdown, edited by Paul Hawken (Fleischmann, 2020). Copyright 2020 by Springer. The figure on the right is an engaging representation of a system within malleable boundaries. "B.O.B" by jdxyw is licensed under CC BY-SA 2.0.

The mapping process on the left in Figure 14, is an example of mapping climate change solution strategies to the Sustainable Development Goals (United Nations, 2015). This process map takes the problem that needs to be addressed - for example, the elimination of poverty - and draws lines to the many climate change actions that address the resolution of poverty. The 
example on the left in Figure 14 is functionally an unintelligible diagram mapping multiple, linear connections from a singular, complex problem, to multiple complex solutions, to multiple complex problems. The simple premise of systems thinking is that pretty much everything is connected, and not in equal parts. Problems and solutions are not balanced equations. There are no delineated or solitary actions that can solve wicked problems, much like the adage that once the cake is baked it is impossible to separate the ingredients back out. In a similar food related analogy, B.O.B. is the outcome of a misguided vegetable and ranch-dressing research experiment, however what B.O.B. adds to the foodie conversation is imagination and creativity, two very critical traits needed to envision pathways to solutions. Also, B.O.B. adds boundaries, yet they are neither fixed nor static. He is not an endless ocean of fluidity, he has some constraints, yet he is malleable and squishy, allowing him to serve as a graphic representation of applied systems-thinking to encompass changing thoughts, ideas, and complex interactive solutions. Applied systems thinking needs to incorporate some of B.O.B.'s organic boundaries for it to effectively address the complexity of designing a campus for learning, wellbeing, and equity.

To design and build a campus learning environment, it is important to define the desired outcomes and then use those as the vision to provide the boundaries for the scope of the work. In project management terms this is called the project charter. The research identified that the charter needs to articulate the desired outcomes intentionally and explicitly, and that those outcomes must be championed and accounted for at every step in the process. In the formal process maps, the project manager role is responsible for ensuring that the final project reflects the outcomes as identified in the charter. However, what the project managers stated was that 
they need a check-off box - a list of exactly what is expected in each project. The project manager role is not suited to identify and incorporate the design principles needed to create a learning environment; the role is to manage the logistics of the project. It is a formal process role charged with specific and linear functions that have many dependencies. Their charge, and how their performance is measured, is to bring in a project on time, on budget, and within scope. This leaves a gap in evaluating the actual quality of the project and its performance with regard to its objectives. The quality of the project is not always immediately apparent, and its performance and impact as a learning environment will change over time. Regardless of the intended outcome, its successes and defects are assumed by the larger university organization, and those are currently reflected in monetized costs of building metrics. The impacts to the learning, wellbeing, and equity are not adequately accounted for, which is why there is a need for post-occupancy assessments to use the data to inform future projects.

B.O.B has an additional feature that can help to frame the principles of designing for human learning, he was created through imagination, innovation, and play. These are design attributes that are easily incorporated in early education spaces, but they diminish to the point of extinction in higher education institutions. B.O.B. is a product of a science experiment gone awry, that is also a valuable lesson for students - to think through the consequences of an experiment or a product. Perhaps the experiments that led to the manufacture of intubation tubes that poisoned infants in the NICU would have had different outcomes if downstream consequences were addressed in a creative and artistic manner.

The role of learning environments is to help our brains absorb and process information, and to create new neural pathways that can expand the ways in which we think. Play, fun, 
creative spaces, and attributes that cause us to pause and ponder, are critical components of our learning environments. Humans learn how to learn through play, observation, and experience. The attributes that enable that in kindergarten classrooms and playgrounds should be carried through to higher education environments.

\section{Recommendations for Research and Practice}

The applied studies undertaken for this dissertation highlighted a need for additional research to inform campus design and identified potential process improvements to enhance the learning environment. The most prominent needs relate to metrics, design processes and organizational relationships.

1. Adopt human metrics to evaluate the impact of the campus environment on learning, wellbeing, and equity. Human metrics are not common in the campus facilities design and operational processes. Impacts to humans are alluded to, but not measured. These cannot be captured until the building is occupied, and these must be captured on a longitudinal scale just like building performance metrics. The facilities conditions assessments utilize longitudinal building performance data to formulate deferred maintenance costs, and the argument is frequently made that the growth in deferred maintenance in a building is correlated to a negative impact on the institution's educational mission (APPA, n.d.). Hence, the general argument is that new or existing buildings with no or smaller deferred maintenance backlogs contribute positively to the educational mission. While this assumption may be true, it is unsubstantiated by data. The higher education facilities sector should be held to the same rigor for human performance data as it is for building performance data. 
2. Include the human metrics in decision making processes. Legislatures, taxpayers, cognizant agencies, parents, and students want to know that the campus is run effectively, and financial inputs are maximized. Attributes such as green roofs, koi ponds, chemical free lawns, cultural dishes in the dining halls, curated art collections, and robot delivery services are not luxury amenities, they are core to the mission of the institution. Do they serve as recruitment tools? Absolutely. Are they unnecessary and frivolous expenses? Absolutely not. The cobenefits of these types of attributes demonstrate a thoughtful, data informed decision-making process, that maximizes every dollar spent. This type of reporting is also emerging in funding decisions, as evidenced by the GAO report on accountability for facilities cost-share in federal grants, as it is a currently unchecked and a rising portion of the cost-share formula (U.S.

Government Accounting Office, 2016). Consistent usage and reporting of human metrics is a sound decision-making input, and it is an emerging practice of which universities should be leading the way.

3. Identify, adopt, and prioritize which attributes of the environment contribute most to student learning outcomes so that those can be prioritized in the campus design process. There are hundreds of attributes that contribute to learning, and some of them cost more than others. To ensure responsible stewardship of resources, and an effective learning environment, it is important to understand where to invest financial resources and maintenance efforts. For example, perhaps color has a significant correlation to learning or wellbeing. The walls are getting painted anyway, so a simple selection of a color that is proven to facilitate good mental health adds nothing to the cost of a project, but contributes in a measurable way to learning, wellbeing, or equity. 
4. Develop training modules for higher education facilities professionals to raise awareness of the uniqueness of learning environment. Not all facilities are created equal or serve the same purpose. While every sector has distinctive purposes, the higher education sector serves some of the most diverse populations and purposes in the world. Higher education campuses include hospitals, vivarium, laboratories, offices, music halls, ice rinks, football stadiums, classrooms, residential complexes, fleets, and dining halls, and they all serve the mission of learning. Mission centric education for facilities professionals is critical to creating an environment that infuses that mission on every square foot of the campus.

5. Socialize concepts before introducing them into processes. This is a key strategy in organizational change management (Schein, 2017). Given the different cultures and organizational structures in higher education, it is likely that different strategies will have to be employed with different populations in order for this to be done effectively (Bergquist \& Pawlak, 2008; Becher \& Trowler, 2001). In a conversation I had with the University Architect, we talked about the effectiveness of tying student learning outcomes to the design principles in our discussions with the Dean of the College of Engineering, as well as the culture at Veterinary Medicine around mental health and self-care (C. McMahan, personal communication, 2021). These discussions were part of our own adaptive organizational system, our informal network in which we reflect and strategize on how to advance the institution's mission and manifest its values in the built environment. Socialization needs to happen in the adaptive organizational system before concepts are introduced into the command control system.

6. Incorporate transdisciplinary planning and design. This is different than a collaborative approach to engagement. A transdisciplinary design and planning process would 
include a process in which a diversity of disciplines and professions would come together to design spaces in which diverse disciplines and professions could work together. This would help bridge the gap between a School's stated value of multi-disciplinary education, and its practice of creating barriers to exclude other majors. A transdisciplinary approach can aid in developing attributes throughout the campus that contribute to learning, wellbeing, and equity. Beautiful bathrooms should not exist only in the School of Human Ecology, pet therapy should be available in the School of Engineering as well as the School of Veterinary Medicine, and the sight and sound of birds should be everywhere on campus, not just at the buildings along the Lakeshore Nature Preserve.

7. Identify, incorporate, and elevate learning outcomes in the facilities design and planning process. The University Architect noted to me that the elevation of student learning outcomes as a principle of design was an effective method to connect the project process to the educational mission (C. McMahan, personal conversation, 2020). Learning outcomes and accreditation requirements are often noted in the facilities condition assessments and advanced design plans as the impetus for needing space remodels or new buildings, yet they are not explicitly identified in the project charter or through the design process. The learning outcomes in particular are a means to ensure that learning is prioritized throughout planning and design for each project, and as a means to take a transdisciplinary approach to campus design. Learning outcomes vary by discipline, but often have similarities that could be aided by a holistic approach to campus design. For example, the collection of building level data could feed up to campus level data and then be made available for researchers and classes. The data could be used to identify and address considerations for space utilization, language translation for 
wayfinding, accommodation services, energy consumption, wi-fi needs, service-dog amenities, or areas of respite. This requires a team of diverse experts and scholars, and an enterprise level approach to campus design.

\section{Conclusion}

Institutions of higher education are incredibly complex, as are the campuses that house them. A holistic approach to the design of campuses could better serve their mission and provide for the adaptability and flexibility needed to accommodate the growing knowledge enterprise. This is evident as we look around our campuses and note the different classroom spaces, residence hall rooms and athletic spaces. Campuses are artifacts that demonstrate the evolution of design principles. These principles continue to evolve, as does the research on how the campus environment impacts humans, animals, and plants. This dissertation looked specifically at the relationship between the environment and human learning, wellbeing, and equity; principles that can be difficult to manifest in a place. The University Architect at the University of Wisconsin-Madison commented that he has been designing buildings for over 30 years, and considers himself an expert, but he now feels that he does not know how to do it. He stated, "I know the process to get us there, but I don't know the answer" (C. McMahan, personal conversation, 2020).

Incorporating societal imperatives as manifestations of the built environment is an emerging field of study and practice. University campuses are ideal places to discover how best to do this. The vibrancy and pace of campuses, with continuous influx of new people and new ideas, makes them the ultimate playground for learning. These applied studies create an opportunity to align the physical attributes of the campus more intentionally with the mission of 
the university. Successfully implementing an organizational systems approach can transform campus building design and maintenance, demonstrate accountability and stewardship of precious resources, while also situating the institution as a leader in applied educational research and facilities management. It will simultaneously send a message to the students and faculty that the institution is committed, innovative, and fiscally responsible in its service to the people of Wisconsin. 


\section{REFERENCES}

Aalbers, R., Dolfsma, W., \& Koppius, O. (2004). On and off the beaten path: How individuals broker knowledge through formal and informal networks. Erasmus Research Institute of Management. Retrieved from https://papers.ssrn.com/sol3/papers.cfm?abstract_id=595004

American Association of University Professors, Special Task Force on Hurricane Katrina and New Orleans Universities. (2007). Hurricane Katrina and New Orleans Universities. Retrieved from https://www.aaup.org/report/report-aaup-special-committee-hurricane-katrina-and-neworleans-universities

Allen, J. (2019, January 20). U-M's \$1.6 billion construction bill is higher than 18 countries' GDP. The Ann Arbor News. Retrieved from https://www.mlive.com/news/ann-arbor/2015/08/ums_16_billion_in_ongoing_con.html

Alvarez, J. L., \& Ferreira, M. A. (1995). Networks versus bureaucracies: The dilemmas of organizations of the future. University of Navarra. Research Paper, (288), 1-24.

Amaratunga, D., \& Baldry, D. (2000). Assessment of facilities management performance in higher education properties. Facilities, 18(7/8), 293 - 301.

American Institute of Architects. (n.d.). Framework for design excellence: Inspiring sustainable, resilient, and inclusive design. Retrieved from https:/www.aia.org/resources/6077668framework-for-design-excellence

Anjard, R. (1998). Process mapping: a valuable tool for construction management and other professionals. Facilities, 16(3/4), 79-81. Retrieved from https://www.emerald.com/insight/content/doi/10.1108/02632779810205611/full/pdf?title=proc ess-mapping-a-valuable-tool-for-construction-management-and-other-professionals 
APPA; Leadership in Educational Facilities (n.d.). Facilities condition assessment. Retrieved from https://www.appa.org/bok/facilities-condition-assessment/\#functionality-assessment

Association for the Advancement of Sustainability in Higher Education. (n.d.). Sustainability Tracking, Assessment \& Rating System. Retrieved from https://stars.aashe.org/

Becher, T., \& Trowler, P. R. (2001). Academic tribes and territories: intellectual enquiry and the culture of disciplines. Philadelphia, PA: Society for Research into Higher Education \& Open University Press.

Berglund, L. (2019). Facilities performance metrics in higher ed: Better serving institutions through impactful dashboards [PowerPoint slides]. EAB. Retrieved from https://www.nationalacademies.org/event/05-082019/docs/DD96C1D8C5F10610F35441670C067AF0697A0F19787E

Bergquist, W. H., \& Pawlak, K. (2008). Engaging the six cultures of the academy (Second edition. ed.). San Francisco: Jossey-Bass.

Bergmann, M., Jahn, T., Knobloch, T., Krohn, W., Pohol, C., \& Schramm, E. (2012). Methods for transdisciplinary research: A primer for practice. Frankfurt: Campus Verlag.

Bernstein, J. H. (2015). Transdisciplinarity: A review of its origins, development, and current issues. Journal of Research Practice, 11(1), Article R1. Retrieved from http://jrp.icaap.org/index.php/jrp/article/view/510/412

Bertalannfy, L. V. (1972). The history and status of general systems theory. Academy of Management Journal, December, 407-426. 
Biazzo, S. (2002). Process mapping techniques and organisational analysis: Lessons from sociotechnical system theory. Business Process Management Journal, 8(1), 42-52. https://doi.org/10.1108/14637150210418629

Biglan, A. (1973). The characteristics of subject matter in different academic areas. Journal of Applied Psychology. Vol 57-3, 195-203. Retrieved from http://web.a.ebscohost.com.ezproxy.library.wisc.edu/ehost/pdfviewer/pdfviewer?vid=1\&sid=7 faf8bce-ec79-4d41-9d6b-f949b7c796ad\%40sdc-v-sessmgr03

Bolman, L. G., \& Deal, T. E. (2013). Reframing organizations: artistry, choice, and leadership (Fifth ed.). San Francisco: Jossey-Bass.

Brown, V. A., Harris, J. A., \& Russell, J. Y. (Eds.). (2010). Tackling wicked problems: Through the transdisciplinary imagination. Abingdon, UK: Earthscan.

Carnegie Classification of Institutions of Higher Education. (n.d.). Basic classifications. Retrieved from http://carnegieclassifications.iu.edu/

Carlson, S. (2012). How the campus crumbles: Colleges face challenges from deferred maintenance. Chronical of Higher Education.

Cash, C. S. (1993). Building condition and student achievement and behavior (Doctoral dissertation, Virginia Tech).

Cash, C. S., \& Twiford, T. (2010). Improving student achievement and school facilities in a time of limited funding. The Connexions Project.

Chrisman, M., Ye, S., Reddy, A., \& Purdy, W. (2020). Assessing sitting and standing in college students using height-adjustable desks. Health Education Journal, 79(6), 735-744. 
Clauson, C. (2018). Planning for diversity, inclusion, and equity. Society for College and University Planner's Planning for Higher Education Journal, 47(1). Retrieved from www.sasaki.com/voices/planning-for-diversity-inclusion-and-equity

Clegg, S., Josserand, E., Mehra, A., \& Pitsis, T. S. (2016). The transformative power of network dynamics: A research agenda. Organization Studies, 37(3), 277-291.

Creswell, J. W., \& Creswell, J. D. (2017). Research design: Qualitative, quantitative, and mixed methods approaches: Sage publications.

Crow, M. M., \& Dabars, W. B. (2015). Designing the NEW American university. Baltimore: Johns Hopkins University Press.

Crowley, T. J. (2000). Causes of climate change over the past 1000 years. Science 289(5477), 270277. doi: $10.1126 /$ science. 289.5477 .270

Damelio, Robert. (1996). The basics of process mapping. New York: Quality Resources.

Darling, David (2019, March 28). 2019-21 Capital Budget Status Report. University of WisconsinMadison Campus Planning Committee. Retrieved from https://d1t7dpw65z191w.cloudfront.net/wp-content/uploads/sites/20/2019/03/19_0328-CPCPresentation.pdf

Demirel, A., Coban, A., Yildirim, S., Dogan, C., Sanci, R., \& Ince, Z. (2016). Hidden toxicity in neonatal intensive care units: Phthalate exposure in very low birth weight infants. Journal of Clinical Research in Pediatric Endocrinology, 8(3), 298-304). doi: 10.4274/jcrpe.3027

Division of Facilities Development, Department of Administration, State of Wisconsin. (n.d.). University of Wisconsin-Madison Project 18H2H, Veterinary Medicine Expansion Project Documents. Madison, WI. 
Division of Facilities Development, Department of Administration, State of Wisconsin. (n.d.). University of Wisconsin-Madison Project 18H3E, Gymnasium-Natatorium Project Documents. Madison, WI.

Division of Facilities Development and Management, Department of Administration, State of Wisconsin. (2020). Sustainability guidelines for capital projects. Madison, WI. Retrieved from https://doa.wi.gov/DFDM_Documents/MasterSpecs/Sustainability/DFDM\%20Sustainability\% 20Guidelines\%20for\%20Capita1\%20Projects\%20-\%20V2\%20-\%20Sept\%202020.pdf

Earthman, G. I. (2002). School facility conditions and student academic achievement. Los Angeles: UCLA Institute for Democracy, Education and Access. Retrieved from https://escholarship.org/uc/item/5sw56439

Evans, T. L. (2015). Transdisciplinary collaborations for sustainability education: Institutional and intragroup challenges and opportunities. Policy Futures in Education, 13(1), 70-96.

Fisher, A. (2021). LSA Building addition cerfied as LEED Gold [Press release]. Retrieved from https://record.umich.edu/articles/lsa-building-addition-certified-as-leed-gold/

Freeman, S., Eddy, S. L., McDonough, M., Smith, M. K., Okoroafor, N., Jordt, H., \& Wenderoth, M. P., (2014). Active learning increases student performance in science, engineering, and mathematics. Proceedings of the National Academy of Sciences, USA, 111, 8410-8415.

Fuller, S. (2016, September 19). Life-cycle cost analysis (LCCA). National Institute of Building Sciences, An Authoritative Source of Innovative Solutions for the Built Environment, 1090.

Gigliotti, R. A. (2020). Sudden shifts to fully online: perceptions of campus preparedness and implications for leading through disruption. Journal of Literacy and Technology, 21(2). 
Golbazi, M., El Danaf, A., \& Aktas, C. B. (2020). Willingness to pay for green buildings: A survey on students' perception in higher education. Energy and Buildings, 216, 109956.

Gulati, R., Dialdin, D. A., \& Wang, L. (2002). Organizational networks. The Blackwell companion to organizations, 281-303.

Habermas, J. (1972). Knowledge and Human Interests, (J. Shapiro, Trans.). Cambridge: Polity Press.

Hawken, P. (2017). Drawdown: The most comprehensive plan ever proposed to reverse global warming. New York, New York: Penguin Books.

Hines, E. W. (1996). Building Condition and Student Achievement and Behavior.

Holmes, K. (2018). Mismatch: How inclusion shapes design. London, England: The MIT Press.

Hunn, B. D. (2010). 35 Years of Standard 90.1. ASHRAE Journal, 52(3), 36-38.

Huron Consulting Group, the American Council on Education \& the Georgia Institute of Technology. (2019). The transformation-ready higher education institution: How leaders can prepare for and promote change [Power Point Slides]. Retrieved from https://www.acenet.edu/Documents/The-Transformation-Ready-Higher-Education-InstitutionHuron-ACE-

Ebook.pdf\#search=How\%20Leaders $\% 20$ can\%20Prepare $\% 20$ for $\% 20$ and $\% 20$ Promote $\% 20$ Cha nge

Institution for Higher Education Policy (Eds.). (2007). College and university ranking systems: Global perspectives and American challenges. Retrieved from http://www.ihep.org/sites/default/files/uploads/docs/pubs/collegerankingsystems.pdf International WELL Building Institute (n.d.). Better buildings to help people thrive. Retrieved from https://www.wellcertified.com/certification/v2/ 
Jackson, M. C. (1982). The nature of 'soft' systems thinking: The work of Churchman, Ackoff and Checkland. Journal of Applied Systems Analysis 9, 17-29. Retrieved from https://www.researchgate.net/profile/Michael_Jackson56/publication/292806531_The_Nature _of_Soft'_Systems_Thinking_The_Work_of_Churchman_Ackoff_and_Checkland/links/5c58 5f23458515a4c757e2ff/The-Nature-of-Soft-Systems-Thinking-The-Work-of-ChurchmanAckoff-and-Checkland.pdf

Jahn, T. (2008). Transdisciplinarity in the practice of research. In Matthias Bergmann/Engelbert Schramm (Hg.) (2008): Transdisziplinäre Forschung. Integrative Forschungsprozesse verstehen und bewerten. Frankfurt/New York: Campus Verlag, 21-37 (English translation, not yet published).

Janks, G., Lockhart, M., \& Travis, A. S. (2012). New metrics for the new normal: rethinking space utilization within the university system of Georgia. Planning for Higher Education, 41(1), 3864.

Jones, N. L. (1991). Essential requirements of the Act: A short history and overview. The Milbank Quarterly, 69, 25-54.

Kaiser, H. H. and Klein, E. (2010). Strategic capital development: The new model for campus investment. Association of Higher Education Facilities Officers: Alexandria, Virginia, 2009. 
Kärnä, S., \& Julin, P. (2015). A framework for measuring student and staff satisfaction with university campus facilities. Quality Assurance in Education. https://www.emerald.com/insight/content/doi/10.1108/QAE-10-20130041/full/html?casa_token=DDjN6Jcw_A4AAAAA:c7rD7UkRpMnN2fJz3hgTKtgiMDpadEi6UFJcgiB_FuRmtiIcSAkkoRg1rNO9dL_zWmmwREurfbplsPnNuEBlL1Fym RGuzKvo-p5yN2tbhvBSBjghwgY

Keeling, R. P., Underhile, R., \& Wall, A. F. (2007). The dynamics of organization in higher education. Liberal Education, Fall, 22-31.

Kerr, C. (2001). The uses of the university: ( $5^{\text {th }}$ ed.). Cambridge, Mass: Harvard University Press.

Kezar, A. J., \& Holcombe, E. M. (2017). Shared leadership in higher education: Important lessons from research and practice. American Council on Eduation \& Center for Policy Research and Strategy: Viewpoints Voices from the Field.

Kim, A. A., Sunitiyoso, Y., \& Medal, L. A. (2019). Understanding facility management decision making for energy efficiency efforts for buildings at a higher education institution. Energy and Buildings, 199, 197-215.

Klepeis, N. E., Nelson, W. C., Ott, W. R., Robinson, J. P., Tsang, A. M., Switzer, P., ... Engelmann, W. H. (2001). The National Human Activity Pattern Survey (NHAPS): a resource for assessing exposure to environmental pollutants. J Expo Anal Environ Epidemiol, 11(3), 231252. doi:10.1038/sj.jea. 7500165

Kok, H. B., Mobach, M. P., \& Omta, O. S. (2011). The added value of facility management in the educational environment. Journal of Facilities Management. 
Küller, R., Ballal, S., Laike, T., Mikellides, B., \& Tonello, G. (2006). The impact of light and colour on psychological mood: a cross-cultural study of indoor work environments. Ergonomics, 49(14), 1496-1507.

Kurt, S., \& Osueke, K. K. (2014). The effects of color on the moods of college students. Retrieved from https://journals.sagepub.com/doi/full/10.1177/2158244014525423

Kwak, Y. H., \& Anbari, F. T. (2009). Analyzing project management research: Perspectives from top management journals. International journal of project management, 27(5), 435-446.

Lawson, S., Sanders, K., \& Smith, L. (2015). Commodification of the information profession: A critique of higher education under neoliberalism. Journal of librarianship and scholarly communication, 3(1).

Lee, H., Kwon, S., \& Lim, J. (2016). A development of a lighting control system based on contextawareness for the improvement of learning efficiency in classrooms. Springer Science+Business Media. doi: 10.1007/s11277-015-2811-6

Leon, R., \& Rossberg, J. (2012). Evolution and Future of Building Codes in the USA. Structural engineering international, 22(2), 265-269.

Lichtenstein, B. B., Uhl-Bien, M., Marion, R., Seers, A., Orton, J. D., \& Schreiber, C. (2006). Complexity leadership theory: An interactive perspective on leading in complex adaptive systems. Retrieved from https://digitalcommons.unl.edu/managementfacpub/8/

Machlup, F. (1962). The production and distribution of knowledge in the United States. Princeton, NJ: Princeton University Press.

Maxwell, J. A., \& Loomis, D. M. (2003). Mixed methods design: an alternative approach in: A Tashakkori and C. Teddlie (Eds), Mixed methods in social and behavioral research. 
McDowell, T., Horn, H., \& Witkowski, D. (2016). Organizational network analysis: Gain insight, drive smart. Deloitte Development LLC, Member of Deloitte Touche Tohmatsu Limited.

McLaughlin, P., \& Faulkner, J. (2012). Flexible spaces... what students expect from university facilities. Journal of Facilities Management.

Mendoza, P. (2010). A Mission of Amenities, Not Education. Academe.

Methorst, J., Rehdanz, K., Mueller, T., Hansjurgens, B., Bonn, A., Bohning-Gaese, K., (2021). The importance of specied diversity for human well-being in Europe. Ecological Economics 181, 106917. https://doi.org/10.1016/j.ecolecon.2020.106917

Meyer, R. J. (1967). Academic freedom in the United States. British Journal of Educational Studies 15(1), 28-39.

Meyerhofer, K. (2021, April 6). Concrete slab falls from third-floor patio of Van Hise Hall on UWMadison campus. Wisconsin State Journal. Retrieved from https://madison.com/wsj/news/local/education/university/concrete-slab-falls-from-third-floorpatio-of-van-hise-hall-on-uw-madison-campus/article_26f86d5b-f58b-5b5a-b1645f2b6459fae3.html

Meyerhofer, K. (2021, February 5). Facing 'financial disaster' from COVID-19, UW System pushes for borrowing ability. Wisconsin State Journal. Retrieved from https://madison.com/wsj/news/local/education/university/facing-financial-disaster-from-covid19-uw-system-pushes-for-borrowing-ability/article_aaa1b472-9a12-5014-85dba19de0d1c095.html

Monaghan, P. (2019, June 16). What the woods do for stressed students. The Chronicle of Higher Education. Retrieved from www.chronicle.com 
NACUBO (2016, January 25). Utilization of classrooms in U.S. Colleges and Universities. Retrieved from https://www.nacubo.org/-/media/Nacubo/Documents/University-UsabilityResources.ashx?1a=en\&hash=632172C65880CBA5B4AEA214A5D0B67AACC71EA6

National Center for Science and Engineering Statistics, National Science Foundation. (2019). Higher education research and development survey fiscal year 2018. Retrieved from http://ncsesdata.nsf.gov/herd/

National Center for Science and Engineering Statistics, National Science Foundation. (2021). Higher education $R \& D$ expenditures, ranked by $R \& D$ expenditures: FYs 2010-19, Table \#23. (Report No. NSF 21-314). Retrieved from https://ncses.nsf.gov/pubs/nsf21314\#general-notes\&datatables

National Research Council. (2000). How people learn: Brain, mind, experience, and school: Expanded edition. National Academies Press.

Nepal, B. (2016). Relationship among school's infrastructure facilities, learning environment and student's outcome. International Journal for Research in Social Science and Humanities Research, 2(5), 44-57.

Nietzel, M. T. (2020, August 13). How universities are increasing the utility of the humanities. Forbes. Retrieved from https://www.forbes.com/sites/michaeltnietzel/2020/08/13/howcolleges-are-trying-to-increase-the-utility-of-the-humanities/?sh=5438944944be

Norris, D., Baer, L., Leonard, J., Pugliese., \& Lefrere, P. (2008). Action analytics: Measuring and improving performance that matters in higher education. EDUCAUSE review 43(1), 42. 
Organisation for Economic Cooperation and Development, Centre for Educational Research and Innovation. (1972). Interdisciplinarity: Problems of teaching and research in universities. (ED 061 895). Retrieved from https://eric.ed.gov/?id=ED061895

Ostherr, K. (2020, May 21). Humanities as essential services. Inside Higher Education. Retrieved from https://www.insidehighered.com/views/2020/05/21/how-humanities-can-be-part-frontline-response-pandemic-opinion

Pettit, E. (2019, November). The professor's views are loathsome, stupid, and ignorant, the Provost said. But he won't be fired. The Chronicle of Higher Education. Retrieved from https://www.chronicle.com/article/The-Professor-s-ViewsAre/247606?cid=wcontentlist_hp_latest

Povey, B. (1998). The development of a best practice business process improvement methodology. Benchmarking for Quality Management and Technology, 5(1), 27-44.

Princeton Review. (n.d.). Green Rating Methodology. Retrieved from https://www.princetonreview.com/college-rankings/green-guide/methodology

Recreation and Wellbeing. (2020). Annual Report 2019-20. Madison, WI: University of WisconsinMadison.

Richardson, G. R., \& Lynes, J. K. (2007). Institutional motivations and barriers to the construction of green buildings on campus. International journal of sustainability in higher education.

Rittel, H. W. J., \& Webber, M. M. (1973). Dilemmas in a general theory of planning. Policy Sciences, 4, 155-169.

Said, E. (1978). Orientalism. New York: Pantheon Books.

Schein, E. H. (2004). Organizational culture and leadership (3rd ed.). San Francisco: Jossey-Bass. 
Schein, E. (2017). Organizational culture and leadership ( $5^{\text {th }}$ ed.). Hoboken, NJ: John Wiley \& Sons, Inc.

Schneider, M. (2002). Do school facilities affect academic outcomes? National Clearinghouse for Educational Facilities.

Senge, Peter M. (1990). The fifth discipline: the art and practice of the learning organization. New York: Doubleday/Currency.

Sharp, L. (2012). Idea Flow. Licensed through Creative Commons.

Sierra Club. (2019). Cool Schools 2020 Methodology. Retrieved from https://www.sierraclub.org/sierra/cool-schools-2020/cool-schools-2020-methodology

Skipper, G. E., \& Williams, J. B. (2012). Failure to acknowledge high suicide risk among veterinarians. Journal of veterinary medical education, 39(1), 79-82.

Song, A. M., Temby, O., Kim, D., Cisneros, A. S., \& Hickey, G. M. (2019). Measuring, mapping and quantifying the effects of trust and informal communication on transboundary collaboration in the Great Lakes fisheries policy network. Global Environmental Change, 54, 6-18.

Sousa, D. (2011). How the brain learns (4 $4^{\text {th }}$ ed.). Corwin, CA: Thousand Oaks.

Stadel, A., Eboli, J., Ryberg, A., Mitchell, J., \& Spatari, S. (2011). Intelligent sustainable design: Integration of carbon accounting and building information modeling. Journal of professional issues in engineering education and practice, 137(2), 51-54.

State Constitution, Constitution of Michigan. (1963). § University of Michigan, Michigan State University, Wayne State Univeristy; controlling boards. Retrieved from http://legislature.mi.gov/doc.aspx?mcl-Article-VIII-5 
State of Michigan (2020). 100 ${ }^{\text {th }}$ Legislature, Senate Bill No. 748, State Appropriations.

http://www.legislature.mi.gov/documents/2019-2020/publicact/pdf/2020-PA-0257.pdf

Suskie, L. (2018). Assessing student learning: A common sense guide (3 ${ }^{\text {rd }}$ ed.). San Francisco, CA: Jossey-Bass.

TCP. (n.d.). The psychological impact of light and color. Retrieved from https://www.tcpi.com/psychological-impact-lightcolor/\#: :text=The\%20Psychological\%20Impact\%20of\%20Light\%20and\%20Color,Commercial\&text $=$ When $\% 20$ light $\% 20$ biologically $\% 20$ impacts $\% 20$ us, better $\% 20$ and $\% 20$ deepe r\%20nights\%20sleep.

Tashakkori, A., \& Teddlie, C. (Eds.). (2010). Mixed methods in social \& behavioral research (2nd ed.). Thousand Oaks, CA: SAGE Publications.

Tashakkori, A., \& Teddlie, C. (2010). Putting the human back in "human research methodology": The researcher in mixed methods research.

Thelin, J. R. (2011). A history of American higher education. Baltimore: The Johns Hopkins University Press.

Tomasi, S. E., Fechter-Leggett, E. D., Edwards, N. T., Reddish, A. D., Crosby, A. E., \& Nett, R. J. (2019). Suicide among veterinarians in the United States from 1979 through 2015. Journal of the American Veterinary Medical Association, 254(1), 104-112.

Tookaloo, A., \& Smith, R. (2015). Post occupancy evaluation in higher education. Procedia engineering, $118,515-521$.

Uhl-Bien, M., \& Arena, M. (2017). Complexity leadership: Enabling people and organizations for adaptability. Organizational Dynamics 46, 9-20. 
Uhl-Bien, M., Marion, R., \& McElvey, B. (2007). Complexity leadership theory: Shifting leadership from the industrial age to the knowledge era. The Leadership Quarterly, 18(4), 298-318.

United Nations. (1987). Report of the world commission on environment and development: Our common future. UN Documents.

United Nations. (2015). Transforming our world: The 2030 agenda for sustainable development. New York: UN Publishing.

United States Department of Education. (n.d.). College Scorecard. https://collegescorecard.ed.gov/school/

United States Government Accounting Office. (2016). NIH biomedical research: Agencies involved in the indirect cost rate-setting process need to improve controls. (GAO-16-616). Retrieved from https://www.gao.gov/products/gao-16-616

University of Michigan (n.d.). Capital planning guidelines. Retrieved from https://www.provost.umich.edu/space/capital/CP_Guidelines.pdf

University of Wisconsin-Madison (2018). Budget in brief. Retrieved from https://chancellor.wisc.edu/content/uploads/2018/02/Budget-in-Brief-2017-18-pdf.pdf University of Wisconsin-Madison (2020). Budget in brief: Budget report 2019-2020: Board of visitors edition. Retrieved from https://budget.wisc.edu/content/uploads/Budget-in-Brief-201920_web.pdf

University of Wisconsin-Madison. (2020). FY 21-23 Capital Budget All Agency Project List. University of Wisconsin-Madison, Division of Facilities Planning \& Management, Project Delivery. (n.d.). Capital planning: Approval process. Retrieved from https://cpd.fpm.wisc.edu/capitalbudget/ 
University of Wisconsin-Madison, Division of Facilities Planning \& Management, Project Delivery (2017). UW-Madison campus design guidelines and standards: An approach to a pleasing composition, rev. August 2017. Retrieved from https://cpd.fpm.wisc.edu/technical-guidelines/

University of Wisconsin-Madison (2012). Office of Space Management: Instructional Space Attributes. Retrieved from https://smoreports.fpm.wisc.edu/Reports/Pages/Report.aspx?ItemPath=\%2fInstructional+Space $\% 2$ fAttrib utes-list

University of Wisconsin-Madison, Office of the Provost, Teaching and Learning. (2016). Learning space improvement report. Retrieved from https://www.wisconsin.edu/news/archive/regentsapprove-outcomes-based-metrics-faculty-workload-policies/

University of Wisconsin-System. (2017, December 7). Regents approve outcomes-based metrics, faculty workload policies. Retrieved from https:/www.wisconsin.edu/news/archive/regentsapprove-outcomes-based-metrics-faculty-workload-policies/

University of Wisconsin-System. (n.d.). Accountability dashboard. Retrieved from https://www.wisconsin.edu/accountability/

University of Wisconsin-System. (2018). Agency Budget Request 2019-2021 Biennium. Retrieved from https://uwm.edu/budget2019-21/wp-content/uploads/sites/246/2018/11/2019-21-285UWS-Budget-Request.pdf

University of Wisconsin-System. (n.d.). 2021-23 Capital Budget Request. Retrieved from https://www.wisconsin.edu/capital-planning/capital-budget/capital-plan/\#2021-23-capitalbudget-request 
University of Wisconsin-System, Board of Regents. (2020). Board of Regents of the University of Wisconsin System, Minutes August 20, 2020. Retrieved from https://www.wisconsin.edu/regents/download/meeting_materials/2020_meeting_materials/mee ting-book---board-of-regents-meeting---august-20,-2020.pdf

Vetterman, R., \& Vernon, C. (Directors), \& Stewart, L. (Producer). (2009). Monsters vs. aliens. [Motion picture]. DreamWorks.

Wang, H. J., Zhao, J. L., \& Zhang, L. J. (2009). Policy-Driven Process Mapping (PDPM): Discovering process models from business policies. Decision Support Systems, 48(1), 267-281.

Wenger, E., McDermott, R., \& Snyder, W. M. (2002). Cultivating communities of practice. Boston, MA: Harvard Business School Press.

Weiman, C., (2014). Large-scale comparison of science teaching methods sends clear message. Proceedings of the National Academy of Sciences, USA, 111, 8319-8320.

Whitchurch, G. G., \& Constantine, L. L. (2009). Systems theory. In Sourcebook of family theories and methods (pp. 325-355). Springer, Boston, MA.

Williams, M. (2001). Problems of knowledge: A critical introduction to epistemology. Oxford, UK: Oxford University Press.

Winterbottom, M., \& Wilkins, A. (2009). Lighting and discomfort in the classroom. Journal of environmental psychology, 29(1), 63-75.

Woodhouse, K. (2015). Lazy rivers and student debt. Inside Higher Ed, 15.

Wright, T. S., \& Wilton, H. (2012). Facilities management directors' conceptualizations of sustainability in higher education. Journal of Cleaner Production, 31, 118-125. 
Zhang, Y., Wang, H., Gao, W., Wang, F., Zhou, N., Kammen, D. M., \& Ying, X. (2019). A survey of the status and challenges of green building development in various countries. Sustainability, 11(19), 5385. 